\title{
LAPLACE OPERATORS AND THE 5 MODULE STRUCTURE OF CERTAIN COHOMOLOGY GROUPS
}

\author{
BY
}

\author{
FLOYD L. WILLIAMS( $\left.{ }^{1}\right)$
}

\begin{abstract}
Let $n$ be the maximal nilpotent ideal of a Borel subalgebra of a complex semisimple Lie algebra 8 . Under the adjoint action $n, 8 / n$, and $n^{\prime}$ (the dual space of $n$ ) are $n$ modules. Laplace operators for these three modules are computed by techniques which extend those introduced by B. Kostant in [6]. The kernels of these operators are then determined and, in view of the existence of a Hodge decomposition, the detailed structure of the first degree cohomology groups of $n$ with coefficients in $n, 8 / n$, and $n^{\prime}$ is obtained. These cohomology groups (spaces) are described, in fact, as completely reducible modules of a Cartan subalgebra of $\boldsymbol{s}$.
\end{abstract}

1. Introduction. Suppose that $\left(\nu, V_{\nu}\right)$ is a finite dimensional irreducible representation of a complex semisimple Lie algebra g. Let $r$ denote conjugation of $g$ with respect to a fixed compact real form of $g$ and let $($,$) denote the (nonde-$ generate) Killing form on g. The equations

$$
\{x, y\}=-(x, \tau y), \bar{x}=-\tau x, x, y \text { in } g,
$$

define a complex inner product $\{$,$\} and *operation on g$.

Given a Lie subalgebra $a$ of $g$ let $d_{\left.\nu\right|_{a}}$ be the coboundary operator associated to the restriction $\left.\nu\right|_{a}$ of $\nu$ to $a_{0} d_{\left.\nu\right|_{a}}$ acts on the cochain complex $\Lambda a^{\prime}$ $\otimes V_{\nu}$, where $a^{\prime}$ is the dual space of $a$. The corresponding Laplace operator $L_{\left.\nu\right|_{a}}$ for the $a$ module $V_{\nu}$ is defined by

$$
L_{\left.\nu\right|_{a}}=d_{\left.\nu\right|_{a}}\left(d_{\left.\nu\right|_{a}}\right)^{*}+\left.\left(d_{\left.\nu\right|_{a}}\right)^{*} d \nu\right|_{a}
$$

for an appropriate inner product on $\Lambda a^{\prime} \otimes V_{\nu}$. The space $\Lambda a^{\prime} \otimes V_{\nu}$ is unitarily equivalent to the space $\Lambda \bar{a} \otimes V_{\nu}$ so we may consider $L_{\left.\nu\right|_{a}}$ as an operator on $\Lambda \bar{a} \otimes V_{\nu}$.

Now we make the additional assumption that the orthocomplement $a^{\perp}$ of $a$ in $g$ is also a Lie subalgebra of $g$. Such subalgebras are called Lie summands of

Received by the editors August 31, 1973.

AMS (MOS) subject classifications (1970). Primary 18H25, 17B10, 22E60.

Key words and phrases. Complex semisimple Lie algebra, Lie algebra cohomology, coboundary operator, Laplace operator, irreducible representation, highest weight.

( $\left.{ }^{1}\right)$ Research supported by NSF grant P-22928. 
g. Then an explicit formula for $L_{\left.\nu\right|_{a}}$, due to Kostant, is available; see Theorem 4.4 of [6]. Kostant has applied this formula in the case where $a$ is the nilradical $\mathfrak{n}$ of a parabolic subalgebra of $g$ and thereby has determined the nature of the cohomology groups $H\left(n, V_{\nu}\right)$; see Theorem 5.14 of [6].

It is significant to bear in mind that the representation of $a$ above (or of $n$ in particular) is the restriction to $a$ of a representation $\nu$ of $g$. Suppose one considers, more generally, an arbitrary subrepresentation $\nu^{a}$ of the restriction $\left.\nu\right|_{a}$; say that $\nu^{a}$ acts on an a subspace $U_{\nu}$ of $V_{\nu}$. Then the following question is prompted: What does the corresponding Laplacian $L_{\nu}$ look like on the space $\Lambda \bar{a} \otimes U_{\nu}$ ? We answer this question in Theorem 3.1.12, the first result of this paper. Thus Theorem 3.1.12 generalizes Theorem 4.4 of [6].

The a subspace $U_{\nu}$ defines a quotient representation $\nu^{a}$ of a on $V_{\nu} / U_{\nu}$. A formula for the corresponding Laplacian $L_{\nu}$ is given by Theorem 3.2.12. We also determine the Laplacian for the contragredient to the adjoint representation of a Lie summand on its dual; see Theorem 3.3.11.

Choose a Cartan subalgebra $\bar{G}$ of $g$ such that $\bar{G}=\bar{G}$ and let $\Delta$ be the set of nonzero roots of $g$ relative to $\mathrm{h}$. With respect to a lexicographic ordering on a real form of $\mathscr{G}$ we can choose a subset $\Delta^{+} \subset \Delta$ of positive roots. Now let $n=$ $\Sigma_{a \in \Delta}+g_{\alpha}$ where the $g_{\beta}, \beta \in \Delta$, are the one dimensional root spaces of $g_{0} n$ is a Lie summand of $g$ since, in fact,

$$
n^{\perp}=\mathfrak{G}+\bar{n}=\bar{G}+\sum_{a \in \Delta^{+}} 9_{-a^{*}}
$$

The application of Theorems 3.1.12, 3.2.12, and 3.3.11 to the case $a=n$ provides, ultimately, an explicit description of the first degree cohomology spaces $H^{1}(n, n), H^{1}(n, g / n), H^{1}\left(n, n^{\prime}\right)$ as completely reducible $h$ modules; see Theorems 5.3.12, 7.5.3, 8.7.13. The dimensions of these spaces depend entirely on the rank of $g(=\operatorname{dim} 5)$. It turns out, moreover, that $H^{1}(\mathfrak{n}, g / n)$ and $H^{1}\left(n, n^{\prime}\right)$ have the same dimension.

Theorem 5.3.12, which describes the space $H^{1}(n, n)$, is a result that was first obtained by Kostant but was never published; cf. the introductory remarks in [6, p. 332]. It is our great pleasure to present Professor Kostant's result in this paper. We also express our sincere gratitude to him for his expert advice and many helpful suggestions which stimulated this research.

2. Laplace operators and Lie algebra cohomology.

2.1 We shall review some of the notation, terminology, and results of Kostant's paper [6].

Suppose that $d, \delta$ are linear operators on a finite dimensional vector space $V$ over any base field. Assume that $d^{2}=\delta^{2}=0$. Under these conditions we say that $d$ and $\delta$ are disjoint if 


$$
d \delta x=0 \text { implies } \delta x=0
$$

and

for any $x$ in $V$.

$$
\delta d x=0 \text { implies } d x=0
$$

Definition 2.1.1 If $d, \delta$ are disjoint, then the operator

$$
L=d \delta+\delta d
$$

is called a Laplace operator (or a Laplacian).

The operators $d, \delta$ define a direct sum decomposition of $V$. More precisely we have

Proposition 2.1.3 If $d, \delta$ are disjoint (as above) then

(i) $\operatorname{Ker} L=\operatorname{Ker} d \cap \operatorname{Ker} \delta$,

(ii) $\operatorname{Im} L=\operatorname{Im} d+\operatorname{Im} \delta$,

(iii) $V=\operatorname{Im} d+\operatorname{Im} \delta+\operatorname{Ker} L$ (direct sum).

The proof, which is elementary, relies very heavily on the disjointness assumption and the finite dimensionality of $V$; see [6]. (iii) is called a Hodge de. composition of $V$.

Since $d^{2}=0$ we can form the derived space $H(V, d)=\operatorname{Ker} d / \operatorname{Im} d$. By (i) $\operatorname{Ker} L \subset \operatorname{Ker} d$.

Corollary 2.1.4 Let $\Gamma: \operatorname{Ker} d \rightarrow H(V, d)$ be the natural homomorphism. Then the restriction of $\Gamma$ to $\operatorname{Ker} L$ is an isomorphism of $\operatorname{Ker} L$ onto $H(V, d): \operatorname{Ker} L$ $\simeq H(V, d)$.

Hence $H(V, d)$ can be computed by determining the zeroes of the Laplacian $L$. This observation will be the basis for computing Lie algebra cohomology in this work. We shall consider the particular case where $V$ is a complex vector space equipped with an inner product. Then it is quite clear that $d$ and its adjoint $d^{*}$ are disjoint. The corresponding Laplacian $L=d d^{*}+d^{*} d$ (with $\delta=d^{*}$ ) is hermitian and the corresponding Hodge decomposition given in (iii) is an orthogonal direct sum decomposition.

2.2 Let $g$ be a complex semisimple Lie algebra. Let $($,$) denote the Killing$ form on g. Choose, once and for all, a compact real form $E$ of Let $\tau$ denote conjugation of $g$ with respect to $E$ :

$$
\tau(x+\sqrt{-1} y)=x-\sqrt{-1} y, \quad x, y \in € .
$$

The equation

$$
\{x, y\}=-(x, \tau y), \quad x, y \in g,
$$

defines a complex inner product $\{$,$\} on g_{0}$ With respect to this inner product the operators $\theta(x): y \rightarrow[x, y], x \in \mathbb{E}, y \in g$, on $g$ are skew-hermitian。There is a 
*operation on 9 defined by

$$
\bar{x}=-7 x, \quad x \in g .
$$

We remark that $\bar{x}$ is written as $x^{*}$ in [6]. Our preference is to use the bar rather than the star. For all $x, y$ in $g$ we have

$$
\overline{(x, y)}=(\bar{x}, \bar{y})
$$

and

$$
\overline{[x, y]}=-[\bar{x}, \bar{y}]
$$

The inner product and *operation on $g$ extend to the exterior algebra $\Lambda \mathrm{g}$ of $g$. Moreover the Killing form extends to a nondegenerate symmetric bilinear form $($, on $\Lambda \mathrm{g}$ :

$$
\left(x_{1} \wedge \ldots \wedge x_{p}, y_{1} \wedge \ldots \wedge y_{q}\right)=\delta_{p q} \operatorname{det}\left(x_{i}, y_{j}\right),
$$

$x_{i}, y_{j} \in g .(),,\{$,$\} , and * are related by the equation$

$$
\{u, v\}=(u, \bar{v}), \quad u, v \in \Lambda g
$$

see [6].

Given $u$ in $\Lambda g$ we define endomorphisms $\epsilon(u), c(u)$ of $\Lambda g$ by

$$
\epsilon(u)_{v}=u \wedge v, \quad(\epsilon(u) v, w)=(v, \iota(u) w)
$$

for all $v, w$ in $\Lambda g$. Thus $\ell(u)=\epsilon(u)^{t}$ is the transpose of $\epsilon(u)$ relative to the nondegenerate form $($,$) on \Lambda g_{0} \epsilon(u)$ is left exterior multiplication by $u$ and $\iota(u)$ is left interior multiplication by $u$. In particular if $u$ is in $g$ then $e(u)$ is given by

$$
\iota(u) x_{1} \wedge \ldots \wedge x_{k}=\sum_{i=1}^{k}(-1)^{j+1}\left(x_{j}, u\right) x_{1} \wedge \ldots \wedge \hat{x}_{j} \wedge \ldots \wedge x_{k},
$$

$x_{j} \in g$, where ' denotes omission. Also $\iota(u)$ is a derivation of degree -1 for $u$ in $g$; see [6].

We shall use the symbol $\theta$ to denote the adjoint representation of $g$ on $\Lambda \mathrm{g}$. Thus $\theta(x)$ is the unique derivation of $\Lambda \mathrm{g}$ of degree 0 which extends the endomorphism $\theta(x): y \rightarrow[x, y], y \in g$, of $g$, where $x \in g . \theta$ is given by the explicit formula

$$
\theta(x) y_{1} \wedge \ldots \wedge y_{k}=\sum_{j=1}^{k} y_{1} \wedge \ldots \wedge\left[x, y_{j}\right] \wedge \ldots \wedge y_{k}, \quad x, y_{j} \in g .
$$

2.3 Now suppose that $\nu$ is a finite dimensional representation of $g$ on a complex vector space $V_{\nu}$. Choose an inner product $\{$,$\} on V_{\nu}$ such that the operators $\nu(k), k \in \mathcal{E}$, are skew-hermitian:

$$
\nu(k)=-\nu(k)^{*}, \quad k \in \mathcal{E} .
$$


This can always be done and $\{$,$\} is in fact unique up to a positive multiple when$ $\nu$ is irreducible. (2.3.1) immediately implies that

$$
\nu(x)^{*}=\nu(\bar{x})
$$

for all $x$ in 9 .

Let $a$ be a Lie subalgebra of $g$ and let $a^{\prime}$ denote the complex dual of $a$. By Lemma 3.4 of [6] there is an algebra is omorphism $\tilde{\eta}$ of $\Lambda \bar{a}$ onto $\Lambda a^{\prime} . \tilde{\eta}$ is defined by the equation

$$
\tilde{\eta}(v)(u)=(u, v)
$$

where $u \in \Lambda a, v \in \Lambda \bar{a} ; \tilde{\eta}$ preserves degrees. The equation

$$
\eta \tilde{\eta}=1_{\Lambda \bar{a}}
$$

defines a monomorphism $\eta: \Lambda a^{\prime} \rightarrow \Lambda g$ whose range is $\Lambda \bar{a}$. The inner product on $\Lambda \mathrm{g}$ restricts to an inner product on $\Lambda \bar{a}$. Therefore, because $\eta$ is $1-1$, there is an inner product $\{$,$\} on \Lambda a^{\prime}$ such that

$$
\eta: \Lambda a^{\prime} \rightarrow \Lambda \bar{a}
$$

is a unitary map onto. Then the equation

$$
\left\{f_{1} \otimes v_{1}, f_{2} \otimes v_{2}\right\}=\left\{f_{1}, f_{2}\right\}\left\{v_{1}, v_{2}\right\}, \quad f_{1}, f_{2} \in \Lambda a^{\prime}, v_{1}, v_{2} \in V_{\nu}
$$

defines an inner product $\{$,$\} on \Lambda a^{\prime} \otimes v_{\nu}$ such that

$$
\eta \otimes 1: \Lambda a^{\prime} \otimes v_{\nu} \rightarrow \Lambda \bar{a} \otimes v_{\nu}
$$

is a unitary map onto.

2.4 Let $\left.\nu\right|_{a}$ denote the restriction of $\nu$ to $\left.a_{0} \nu\right|_{a}$ is a representation of $a$ on $V_{\nu}$. Hence we can form the cohomology groups $H^{k}\left(a, V_{\nu}\right), k=0,1,2, \cdots$, with coefficients in $V_{\nu}$. We recall, briefly, how this is done; see [3]. Define $\Lambda^{k}\left(a, V_{\nu}\right)=$ complex vector space of $k$ linear alternating maps

$$
f: a \times \cdots \times a \rightarrow v_{\nu}
$$

Thus $f\left(x_{1}, \cdots, x_{i}, \cdots, x_{j}, \cdots, x_{k}\right)=0$ for $x_{i}=x_{j}, i \neq j, x_{i} \in a$. If $k=0$ we set $\Lambda^{0}\left(a, V_{\nu}\right)=V_{\nu}$. Elements of $\Lambda^{k}\left(a, V_{\nu}\right)$ are called $k$ dimensional cochains.

Define

$$
d=d_{\nu, k}=d_{k}: \Lambda^{k}\left(a, V_{\nu}\right) \rightarrow \Lambda^{k+1}\left(a, V_{\nu}\right)
$$

by

$$
(d f)\left(x_{1}, \ldots, x_{k+1}\right)=\sum_{i=1}^{k+1}(-1)^{i+1} \nu\left(x_{i}\right) f\left(x_{1}, \ldots, \hat{x}_{i}, \ldots, x_{k+1}\right)
$$

$$
+\sum_{i<j}(-1)^{i+j} f\left(\left[x_{i}, x_{j}\right], x_{1}, \ldots, \hat{x}_{i}, \ldots, \hat{x}_{j}, \ldots, x_{k+1}\right)
$$


$x_{i} \in a, f \in \Lambda^{k}\left(a, V_{\nu}\right)$. If $k=0$, then $d: V_{\nu} \rightarrow \Lambda^{1}\left(a, V_{\nu}\right)$ is defined by

$$
(d v)(x)=\nu(x)_{v}, \quad x \in a, v \in V_{\nu}
$$

Of course $\Lambda^{1}\left(a, V_{\nu}\right)$ is the space of linear maps from a to $V_{\nu} \cdot d_{k}$ is called the $k$ th dimensional coboundary operator. We have $d^{2}=0$; i.e. $d_{k+1} d_{k}=0$. Setting $d_{-1}=0$ we define

$$
H^{k}\left(a, V_{\nu}\right)=\operatorname{Ker} d_{k} / \operatorname{Im} d_{k-1}, \quad H^{0}\left(a, V_{\nu}\right)=\operatorname{Ker} d_{0} .
$$

Therefore $H^{0}\left(a, V_{\nu}\right)=\left\{v \in V_{\nu} \mid \nu(x) v=0 \forall x \in a\right\}$ is the space of invariants of the a module $V_{\nu} \cdot H^{k}\left(a, V_{\nu}\right)$ is the familiar $k$ th dimensional cohomology group of $a$ with coefficients in the a module $V_{\nu}$.

There is a unique vector space isomorphism $\gamma$ of $\Lambda^{k} a^{\prime} \otimes V_{\nu}$ onto $\Lambda^{k}\left(a, V_{\nu}\right)$ such that

$$
\left(\gamma\left(f_{1} \wedge \cdots \wedge f_{k} \otimes v\right)\right)\left(x_{1}, \cdots, x_{k}\right)=\left(\operatorname{det} f_{i}\left(x_{j}\right)\right) v
$$

for all $f_{i} \in a^{\prime}, x_{j} \in a, v \in V_{\nu}$. In particular $\Lambda^{k}\left(a, V_{\nu}\right)=0$ for $k>\operatorname{dim}_{\mathrm{C}} a$. Moreover we can consider the coboundary operator

$$
d_{\left.\nu\right|_{a}}=\sum_{k=0}^{\operatorname{dim} a} \bigoplus d_{k}
$$

as an operator on $\Lambda a^{\prime} \otimes V_{\nu}$. Since $d_{\left.\nu\right|_{a}}^{2}=0$ and since we have an inner product on $\Lambda a^{\gamma} \otimes V_{\nu}$ (see 2.3) we can form the Laplacian

$$
L_{\left.\nu\right|_{a}}=d_{\left.\nu\right|_{a}}\left(d_{\left.\nu\right|_{a}}\right)^{*}+\left(d_{\left.\nu\right|_{a}}\right)^{*} d_{\left.\nu\right|_{a}}
$$

on $\Lambda a^{\prime} \otimes V_{\nu}$. As we have observed earlier a consequence of the Hodge decomposition is the following ideal formula:

$$
H^{k}\left(a, v_{\nu}\right)=\left\{x \in \Lambda^{k} a^{\prime} \otimes V_{\nu} \mid L_{\left.\nu\right|_{a}} x=0\right\} \text {. }
$$

Up to this point $a$ has been an arbitrary Lie subalgebra of $g$. If one assumes moreover that $a$ is a Lie summand-this means that $a^{\perp}$ is also a Lie subalgebra of $g$-then $L_{\left.\nu\right|_{a}}$ can be computed rather explicitly. The result, due to Kostant, is

Theorem 2.4.7 Suppose $a$ is a Lie summand of $g$ and suppose $\left\{z_{1}, \cdots, z_{m}\right\}$, $\left\{z_{m+1}, \cdots, z_{n}\right\}$ are orthonormal bases for $a, a^{\perp}$ respectively; $m=\operatorname{dim}_{C} a, n=$ $\operatorname{dim}_{C} g$. Then

$$
\begin{aligned}
(\eta \otimes 1) L_{\left.\nu\right|_{a}}(\eta \otimes 1)^{-1} & =1 \otimes C^{\nu}+\sum_{i=1}^{m} \theta \otimes \nu\left(\bar{z}_{i}\right) \theta \otimes \nu\left(z_{i}\right) \\
& -\sum_{j=m+1}^{n} \theta \otimes \nu\left(\bar{z}_{j}\right) \theta \otimes \nu\left(z_{j}\right)
\end{aligned}
$$


where $C^{\nu}=\sum_{i=1}^{n} \nu\left(\bar{z}_{i}\right) \nu\left(z_{i}\right)$ is the Casimir operator of $\nu$ and $\eta \otimes 1$ is the unitary map of $\Lambda a^{\prime} \otimes V_{\nu}$ onto $\Lambda \bar{a} \otimes V_{\nu}$ given in (2.3.6).

Thus on $\Lambda \bar{\alpha} \otimes V_{\nu} L_{\left.\nu\right|_{a}}$ is expressed in terms of the adjoint representation $\theta$ and the representation $\nu$; see Theorem 4.4 of [6].

2.5 The representation of the Lie summand $a$ in Theorem 2.4 .7 is the restriction to $a$ of a representation $\nu$ of $g$. This raises a natural question: Can one compute the Laplacian, anore generally, for an arbitrary subrepresentation of the restriction $\left.\nu\right|_{a}$ ? We shall settle this question presently. Prior to this it is convenient to introduce additional operators and some formulas which, incidently, facilitate the proof of Theorem 2.4.7.

Again we suppose $a$ is a Lie subalgebra of $g$ and $\nu$ is a finite dimensional representation of $g$; $a$ need not be a Lie summand.

Corresponding to the trivial representation of $a$ there is the coboundary operator on $\Lambda a^{\prime}$ which we shall denote by $d$. The boundary operator $\partial$ on $a$ is defined, uniquely, by the formula

$$
(d f)(x)=-f(\partial x)
$$

for all $x \in \Lambda a, f \in(\Lambda a)^{\prime}$. In particular

$$
\begin{aligned}
f\left(\partial x_{1} \wedge \ldots \wedge x_{k}\right)=-(d f)\left(x_{1} \wedge \ldots \wedge x_{k}\right) & \\
(\operatorname{see}(2.4 .1)) & =-\sum_{i<j}(-1)^{i+j} f\left(\left[x_{i}, x_{j}\right] \wedge x_{1} \wedge \cdots \wedge \hat{x}_{i} \wedge \cdots \wedge \hat{x}_{j} \wedge \cdots \wedge x_{k}\right) \\
& =f\left(\sum_{i<j}(-1)^{i+j+1}\left[x_{i}, x_{j}\right] \wedge x_{1} \wedge \cdots \wedge \hat{x}_{i} \wedge \cdots \wedge \hat{x}_{j} \wedge \cdots \wedge x_{k}\right)
\end{aligned}
$$

for all $f$ in $(\Lambda a)^{\prime}$. Hence

$$
\begin{aligned}
\partial x_{1} \wedge & \ldots \wedge x_{k} \\
& =\sum_{i<j}(-1)^{i+j+1}\left[x_{i}, x_{j}\right] \wedge x_{1} \wedge \ldots \wedge \hat{x}_{i} \wedge \ldots \wedge \hat{x}_{j} \wedge \ldots \wedge x_{k}
\end{aligned}
$$

for $x_{i} \in a$. Taking $a=g$ we see that the boundary operator on a subalgebra $a$ of $g$ is the restriction to $a$ of the boundary operator on $g$. Because of this we shall denote the boundary operator on $g$ by the same symbol $\partial$.

$\Lambda g$ and $\Lambda g^{\prime}$ can be identified (as algebras in fact) by means of the Killing form. Then one has

$$
-\partial^{t}=\text { coboundary operator of } g .
$$

Here we recall that the transpose is taken with respect to the Killing form on $\Lambda \mathrm{g}$; see (2.2.5). Following Kostant, we denote the endomorphism - $\partial^{t}$ of $\Lambda g$ by $c$.

Now suppose $\left\{z_{1}, \cdots, z_{m}\right\},\left\{z_{m+1}, \cdots, z_{n}\right\}$ are orthonormal bases for $a, a^{\perp}$ 
respectively. Then there are endomorphisms $d_{a, 1}, d_{a, 2}, d_{a, \nu}$ and $c_{\nu}$ of $\Lambda g \otimes V_{\nu}$ (which are well defined for any subspace $a$ of $g$ ) given by

$$
d_{a, 1}=d_{a} \otimes 1
$$

where

$$
\begin{aligned}
d_{a} & =\frac{1}{2}\left(\sum_{i=1}^{m} \epsilon\left(\bar{z}_{i}\right) \theta\left(z_{i}\right)-\sum_{j=m+1}^{n} \epsilon\left(\bar{z}_{j}\right) \theta\left(z_{j}\right)\right), \\
d_{a, 2} & =\sum_{i=1}^{m} \epsilon\left(\bar{z}_{i}\right) \otimes \nu\left(z_{i}\right), \\
d_{a, \nu} & =d_{a, 1}+d_{a, 2}, \\
c_{\nu} & =c \otimes 1+c_{2},
\end{aligned}
$$

where $c_{2}=\Sigma_{i=1}^{n} \epsilon\left(\bar{z}_{i}\right) \otimes \nu\left(z_{i}\right)$. The proof of Theorem 2.4 .7 depends on establishing the following formulas:

$$
\begin{aligned}
& (\eta \otimes 1) d_{\left.\nu\right|_{a}}(\eta \otimes 1)^{-1}=d_{a, \nu} \\
& (\eta \otimes 1)\left(d_{\left.\nu\right|_{a}}\right)^{*}(\eta \otimes 1)^{-1}=c_{\nu}^{*}
\end{aligned}
$$

where (2.5.5) is valid only when $a$ is also a Lie summand; see Lemma 4.1 and Lemma 4.2 in [6].

Germane to the proof of Theorem 2.4 .7 also is the following more general observation.

Proposition 25.7 Let $\mathrm{g}$ be a semisimple Lie algebra (over $\mathrm{C}$ ) and let a be a Lie subalgebra of g. Let $\mu$ be a finite dimensional $\Lambda$ representation of a on a $\mathbf{C}$ vector space $U_{\mu}$. Suppose $\left\{z_{1}, \cdots, z_{m}\right\},\left\{z_{1}^{\prime}, \cdots, z_{m}^{\prime}\right\}$ are dual bases for $a, a^{\prime}$. Then the coboundary operator $d_{\mu}$ on $\Lambda_{a^{\prime}} \otimes U_{\mu}$ is given by

$$
d_{\mu}=\sum_{i=1}^{m} \epsilon\left(z_{i}^{\prime}\right) \otimes \mu\left(z_{i}\right)+d \otimes 1
$$

where $d$ is the coboundary on $\Lambda a^{\prime}$ (corresponding to the trivial representation of $a$ ).

See [7]. In particular

$$
d_{\left.\nu\right|_{a}}=\sum_{i=1}^{m} \epsilon\left(z_{i}^{\prime}\right) \otimes \nu\left(z_{i}\right)+d \otimes 1
$$

Proposition 2.5.9 $\iota(u)^{*}=\epsilon(\bar{u})$ for $u$ in $\Lambda \mathrm{g}$.

See 3.9 .3 in [6]. 
3. The computation of Laplace operators for submodules, quotient modules, and contragredient modules.

3.1 We retain the notation established in $\$ 2$; assume $a$ is a Lie summand of g. Now suppose that $U$ is a subspace of $V_{\nu}$ which is $\nu(a)$ invariant. Then there is a representation $\nu^{a}$ of $a$ on $U$ given by

$$
\nu^{a}(x)=\left.\nu(x)\right|_{U}, \quad x \in a \text {. }
$$

$\nu^{a}$ is a subrepresentation of the restriction $\left.\nu\right|_{a}$. Notice the following simple fact:

Proposition 3.1.2 $U^{\perp}$ is $\nu(\bar{a})$ invariant.

Proof. If $x \in a, u \in U, u^{\perp} \in U^{\perp}$ are arbitrary, then by (2.3.2)

$$
\left\{\nu(\bar{x}) u^{\perp}, u\right\}=\left\{\nu(x)^{*} u^{\perp}, u\right\}=\left\{u^{\perp}, \nu(x) u\right\}=0 \text { : }
$$

- since $U$ is $\nu(a)$ invariant.

For the record we state the following, equally simple,

Proposition 3.1.3 Let $V$ be a finite dimensional complex inner product space. Let $T$ be a linear operator on $V$, let $W$ be a subspace of $V$, and let $P$ be the orthogonal projection of $V$ onto $W$. Then $W$ is $T$ invariant if and only if PTP $=T P$. If $W$ is $T$ invariant then $\left(\left.T\right|_{W}\right)^{*}=\left.P T^{*}\right|_{W}$.

As in 2.4 let $d_{\nu^{a}}$ denote the coboundary operator corresponding to the representation $\nu^{a}$ of $a$ on $U$ and let

$$
L_{\nu^{a}}=d_{\nu^{a}}\left(d_{\nu^{a}}\right)^{*}+\left(d_{\nu^{a}}\right)^{*} d \nu_{\nu^{a}}
$$

be the Laplace operator defined by $\nu^{a}$ (the inner product on $\Lambda a^{\prime} \otimes V_{\nu}$ restricts to an inner product on $\left.\Lambda a^{\prime} \otimes U\right)$. In this section we shall find a formula for $L_{\nu^{a}}$ acting on the space (cohomology complex) $\Lambda \bar{a} \otimes U$.

Let $P$ be the orthogonal projection of $V_{\nu}$ onto $U$. Then $1 \otimes P$ is the orthogonal projection of $\Lambda a^{\prime} \otimes V$ onto $\Lambda a^{\prime} \otimes U$. Let $\left\{z_{1}, \cdots, z_{m}\right\},\left\{z_{m+1}, \cdots, z_{n}\right\}$ be orthonormal bases for $a, a^{\perp}$ and let $\left\{z_{1}^{\prime}, \cdots, z_{m}^{\prime}\right\}$ be a basis of $a^{\prime}$ dual to $\left\{z_{1}, \cdots, z_{m}\right\}$. By Proposition 2.5 .7

$$
d_{\nu^{a}}=\sum_{i=1}^{m} \epsilon\left(z_{i}^{\prime}\right) \otimes \nu^{a}\left(z_{i}\right)+d \otimes 1 .
$$

Hence $\Lambda a^{\prime} \otimes U$ is $d_{\left.\nu\right|_{a}}$ invariant and

$$
d_{\nu^{a}}=\left(\left.d_{\left.\nu\right|_{a}}\right|_{\Lambda a^{\prime} \otimes U}\right.
$$

Proposition 3.1.3 implies

$$
\left(d_{\nu}\right)^{*}=\left.(1 \otimes P)\left(d_{\left.\nu\right|_{a}}\right)^{*}\right|_{\mathbf{\Lambda} a^{\prime} \otimes U}
$$


By (3.1.4) we have on $\Lambda a^{\prime} \otimes U$

$$
\begin{aligned}
L_{\nu^{a}} & =d_{\left.\nu\right|_{a}}(1 \otimes P)\left(d_{\left.\nu\right|_{a}}\right)^{*}+(1 \otimes P)\left(d_{\left.\nu\right|_{a}}\right)^{*} d_{\left.\nu\right|_{a}} \\
& =(1 \otimes P)\left(d_{\left.\nu\right|_{a}}(1 \otimes P)\left(d_{\left.\nu\right|_{a}}\right)^{*}+\left(d_{\left.\nu\right|_{a}}\right)^{*} d_{\left.\nu\right|_{a}}\right),
\end{aligned}
$$

since $\Lambda a^{\prime} \otimes U$ is $d_{\left.\nu\right|_{a}}$ invariant,

$$
\begin{aligned}
& =(1 \otimes P)\left(d_{\left.\nu\right|_{a}}\left(d_{\left.\nu\right|_{a}}\right)^{*}+\left(d_{\left.\nu\right|_{a}}\right)^{*} d_{\left.\nu\right|_{a}}\right)+(1 \otimes P) d_{\left.\nu\right|_{a}}\left[1 \otimes P,\left(d_{\left.\nu\right|_{a}}\right)^{*}\right] \\
& =(1 \otimes P) L_{\left.\nu\right|_{a}}+(1 \otimes P) d_{\left.\nu\right|_{a}}\left[1 \otimes P,\left(d_{\left.\nu\right|_{a}}\right)^{*}\right]
\end{aligned}
$$

by (2.4.5). Let

$$
E=(1 \otimes P) d_{\left.\nu\right|_{a}}\left[1 \otimes P,\left(d_{\left.\nu\right|_{a}}\right)^{*}\right]
$$

The "error" term $E$ measures, somehow, the extent to which $\left(d_{\left.\nu\right|_{a}}\right)^{*}$ fails to leave $\Lambda a^{\prime} \otimes U$ invariant. Conjugating by $(\eta \otimes 1)$, we get

$$
(\eta \otimes 1) L_{\nu a}(\eta \otimes 1)^{-1}
$$

$$
=(1 \otimes P)(\eta \otimes 1) L_{\left.\nu\right|_{a}}(\eta \otimes 1)^{-1}+(\eta \otimes 1) E(\eta \otimes 1)^{-1}
$$

where

$$
\begin{aligned}
(\eta \otimes 1) E(\eta \otimes 1)^{-1} & =(1 \otimes P)(\eta \otimes 1) d_{\left.\nu\right|_{a}}(\eta \otimes 1)^{-1}\left[1 \otimes P,(\eta \otimes 1)\left(d_{\left.\nu\right|_{a}}\right)^{*}(\eta \otimes 1)\right] \\
& =(1 \otimes P) d_{a, \nu}\left[1 \otimes P, c_{\nu}^{*}\right]
\end{aligned}
$$

by $(2.5 .5)$ and $(2.5 .6)$. Now

$$
c_{\nu}^{*}=c^{*} \otimes 1+\sum_{i=1}^{m} \iota\left(z_{i}\right) \otimes \nu\left(\bar{z}_{i}\right)
$$

on $\Lambda \bar{a} \otimes V_{\nu}$ by (2.5.4), Proposition 2.5.9 and the fact that

$$
\iota\left(a^{\perp}\right) \bar{a}=0
$$

see (2.2.8). Therefore on $\Lambda \bar{a} \otimes U$

$$
\begin{aligned}
(1 \otimes P) d_{a, \nu}\left[1 \otimes P, c_{\nu}^{*}\right]= & (1 \otimes P) d_{a, \nu} \sum_{i=1}^{m} \iota\left(z_{i}\right) \otimes(P-1) \nu\left(\bar{z}_{i}\right) \\
= & (1 \otimes P) \sum_{i=1}^{m} d_{a} \iota\left(z_{i}\right) \otimes(P-1)_{\nu}\left(\bar{z}_{i}\right) \\
& +(1 \otimes P) \sum_{j=1}^{m} \sum_{i=1}^{m} \epsilon\left(\bar{z}_{j}\right) \iota\left(z_{i}\right) \otimes \nu\left(z_{j}\right)(P-1)_{\nu}\left(\bar{z}_{i}\right),
\end{aligned}
$$

by $(2.5 .4)$,

$$
=\sum_{j=1}^{m} \sum_{i=1}^{m} \epsilon\left(\bar{z}_{j}\right) \iota\left(z_{i}\right) \otimes P \nu\left(z_{j}\right)(P-1) \nu\left(\bar{z}_{i}\right)
$$


Putting the pieces together, see (3.1.9), we have proved

Theorem 3.2.12 Suppose a is a Lie summand of g. Let $U$ be a subspace of the g module $V_{\nu}$ such that $\nu(a) U \subset U$ and let $\nu^{a}$ be the subrepresentation of $\nu l_{a}$ defined by the a module $U$. Then the Laplacian $L_{\nu^{a}}$ corresponding to the representation $\nu^{a}$ of a on $U$ is given by

$$
(\eta \otimes 1) L_{\nu^{a}}(\eta \otimes 1)^{-1}=\left(1 \otimes P^{U}\right)(\eta \otimes 1) L_{\left.\nu\right|_{a}}(\eta \otimes 1)^{-1}+E
$$

on $\Lambda \bar{a} \otimes U$ where $(\eta \otimes 1) L_{\left.\nu\right|_{a}}(\eta \otimes 1)^{-1}$ (the Laplacian for the representation $\nu l_{a}$ of $a$ on $V_{\nu}$ ) is given by Theorem 2.4.7,

$$
E=\sum_{j=1}^{m} \sum_{i=1}^{m} \epsilon\left(\bar{z}_{j}\right) \iota\left(z_{i}\right) \otimes P^{U} \nu\left(z_{j}\right)\left(P^{U}-1\right) \nu\left(\bar{z}_{i}\right),
$$

$\left\{z_{1}, \cdots, z_{m}\right\}$ is an orthonormal basis of $a$, and $P^{U}$ is the orthogonal projection of $V_{\nu}$ onto $U$.

Note if $U=V_{\nu}$, in particular, then $P=1$ so $E=0$ and Theorem 3.1.12 reduces to Kostant's Theorem 2.4.7.

3.2 Again we suppose that $U$ is a subspace of $V_{\nu}$ which is $\nu(a)$ invariant. Then in addition to the subrepresentation $\nu^{a}$ of $a$ on $U$ there is a quotient representation $\nu^{a}$ of $a$ on $V_{\nu} / U$ :

$$
\nu^{a}(x)(v+U)=\nu(x) v+U
$$

for $x \in a, v \in V$. We shall compute the Laplacian $L_{v^{a}}$ corresponding to the representation $\nu^{a}$. This time let $P$ denote the orthogonal projection of $V$ onto $U^{\perp}$. The map

$$
T: v+U \rightarrow P v, \quad v \in V,
$$

is a well-defined vector space isomorphism of $V_{\nu} / U$ onto $U^{\perp}$.

Proposition 3.2.2 $T \nu^{a}(x) T^{-1}=\left.P \nu(x)\right|_{U^{\perp}}$ for all $x$ in $a$.

Proof. Let $x \in a, u^{\perp} \in U^{\perp}$ be arbitrary. Then $u^{\perp}+U \in V_{\nu} / U$ and $T\left(u^{\perp}+U\right)=$ $P u^{\perp}=u^{\perp}$. Hence

$$
T \nu^{a}(x) T^{-1} u^{\perp}=T \nu^{a}(x)\left(u^{\perp}+U\right)=T\left(\nu(x) u^{\perp}+U\right)=P \nu(x) u^{\perp} .
$$

From now on then we shall consider $\nu^{a}$ as the representation of a on $U^{\perp}$ given by Proposition 3.2.2; i.e.

$$
\nu^{a}(x)=\left.P \nu(x)\right|_{U^{\perp}}
$$

The coboundary operator $d_{\nu^{a}}$ on $\Lambda a^{\prime} \otimes U^{\perp}$ for the representation $\nu^{a}$ is given by Proposition 2.5.7: 


$$
d_{\nu^{a}}=\sum_{i=1}^{m} \epsilon\left(z_{i}^{\prime}\right) \otimes \nu^{a}\left(z_{i}\right)+d \otimes 1
$$

$$
=(1 \otimes P)\left(\sum_{i=1}^{m} \epsilon\left(z_{i}^{\prime}\right) \otimes \nu\left(z_{i}\right)+d \otimes 1\right) ;
$$

i.e.

(3.2.5)

$$
d_{\nu^{a}}=\left.(1 \otimes P)\left(d_{\left.\nu\right|_{a}}\right)\right|_{\Lambda a \prime \otimes U^{1 \cdot P}}
$$

Now $(1 \otimes P) d_{\left.\nu\right|_{a}}$ leaves $\Lambda a^{\prime} \otimes U^{\perp}$ invariant of course so, by Proposition 3.1.3 and by (3.2.5),

$$
\left(d_{\nu^{a}}\right)^{*}=(1 \otimes P)\left(\left.d_{\left.\nu\right|_{a}}\right|_{\Lambda_{a^{\prime}} \otimes U^{\perp}}\right.
$$

On the other hand we claim that $\left(d_{\left.\nu\right|_{a}}\right)^{*}$ leaves $\Lambda a^{\prime} \otimes U^{\perp}$ invariant. Indeed

$$
\left(d_{\left.\nu\right|_{a}}\right)^{*}=\sum_{i=1}^{-m} \epsilon\left(z_{i}^{\prime}\right)^{*} \otimes \nu\left(\bar{z}_{i}\right)+d^{*} \otimes 1
$$

by (2.5.8) and (2.3.2). But $z_{i} \in$ a and $U^{\perp}$ is $\nu(\bar{a})$ invariant by Proposition 3.1.2. From (3.2.6) we deduce that

$$
'\left(d_{\nu^{a}}\right)^{*}=\left.\left(d_{\left.\nu\right|_{a}}\right)^{*}\right|_{\Lambda a \cdot \otimes U^{\perp}}
$$

The Laplacian $L_{\nu^{a}}$ corresponding to the representation $\nu^{a}$ is given on $\Lambda a^{\prime} \otimes U^{\perp}$ by

$$
\begin{aligned}
L_{\nu^{\mathrm{a}}} & =d_{\nu^{\mathrm{a}}}\left(d_{\nu^{\mathrm{a}}}\right)^{*}+\left(d_{\nu^{\mathrm{a}}}\right)^{*} d_{\nu^{\mathrm{a}}} \\
& =(1 \otimes P) d_{\left.\nu\right|_{\mathrm{a}}}\left(d_{\left.\nu\right|_{\mathrm{a}}}\right)^{*}+\left(d_{\left.\nu\right|_{\mathrm{a}}}\right)^{*}(1 \otimes P) d_{\left.\nu\right|_{\mathrm{a}}} \\
& =(1 \otimes P)\left(d_{\left.\nu\right|_{\mathrm{a}}}\left(d_{\left.\nu\right|_{\mathrm{a}}}\right)^{*}+\left(d_{\left.\nu\right|_{\mathrm{a}}}\right)^{*} d_{\left.\nu\right|_{\mathrm{a}}}\right)+(1 \otimes P)\left[\left(d_{\left.\nu\right|_{\mathrm{a}}}\right)^{*}, 1 \otimes P\right] d_{\left.\nu\right|_{\mathrm{a}}} \\
& =(1 \otimes P) L_{\left.\nu\right|_{\mathrm{a}}}+E
\end{aligned}
$$

where

$$
E=(1 \otimes P)\left[\left(d_{\left.\nu\right|_{a}}\right)^{*}, 1 \otimes P\right] d_{\left.\nu\right|_{a}}
$$

$$
(\eta \otimes 1) E(\eta \otimes 1)^{-1}=(1 \otimes P)\left(c_{\nu}^{*}(1 \otimes P)-c_{\nu}^{*}\right) d_{a, \nu},
$$

by (2.5.5) and (2.5.6),

by (3.1.10),

$$
=\left(\sum_{i=1}^{m} \iota\left(z_{i}\right) \otimes P \nu\left(\bar{z}_{i}\right) P-\sum_{i=1}^{m} \iota\left(z_{i}\right) \otimes P \nu\left(\bar{z}_{i}\right)\right) d_{\mathrm{a}, \nu},
$$




$$
=\sum_{i=1}^{m} \sum_{j=1}^{m} \iota\left(z_{i}\right) \epsilon\left(\bar{z}_{j}\right) \otimes P \nu\left(\bar{z}_{i}\right)(P-1) \nu\left(z_{j}\right),
$$

by (2.5.4). On the other hand since

$$
\epsilon(x) \iota(y)+\iota(y) \epsilon(x)=(x, y) 1
$$

for $x, y \in g$, where $1 \in \Lambda \mathrm{g}$, as is immediately verified, we have proved (see (3.2.8) and (3.2.10)):

Theorem 3.2.12 Suppose a is a Lie summand of g. Let $U$ be a subspace of the g module $V_{\nu}$ such that $\nu(a) U \subset U$ and let $\nu^{a}$ be the quotient representation of a on $V_{\nu} / U$. Then the Laplacian $L_{\nu^{a}}$ corresponding to the representation $\nu^{a}$ is given by

$$
(\eta \otimes 1) L_{\nu^{a}}(\eta \otimes 1)^{-1}=\left(1 \otimes P^{U^{\perp}}\right)(\eta \otimes 1) L_{\left.\nu\right|_{a}}(\eta \otimes 1)^{-1}+E
$$

on $\Lambda \bar{a} \otimes U^{\perp}$ where $(\eta \otimes 1) L_{\left.\nu\right|_{a}}(\eta \otimes 1)^{-1}$ (the Laplacian for the representation $\nu l_{\text {a }}$ of a on $V_{\nu}$ ) is given by Theorem 2.4.7,

$$
\begin{aligned}
E= & \sum_{j=1}^{m} \sum_{i=1}^{m} \epsilon\left(\bar{z}_{j}\right) \iota\left(z_{i}\right) \otimes P^{U^{\perp}} \nu\left(\bar{z}_{i}\right)\left(1-P^{U^{\perp}}\right) \nu\left(z_{j}\right) \\
& +1 \otimes \sum_{j=1}^{m} P^{U^{\perp}} \nu\left(\bar{z}_{j}\right)\left(P^{U^{\perp}}-1\right) \nu\left(z_{j}\right),
\end{aligned}
$$

$\left\{z_{1}, \cdots, z_{m}\right\}$ is an orthonormal basis of $a$, and $P^{U^{\perp}}$ is the orthogonal projection of $V_{\nu}$ onto $U^{\perp}$.

3.3 In this section we shall give a formula for the Laplace operator $L_{\theta^{a^{\prime}}}$ where $\theta^{a^{\prime}}$ is the co-adjoint representation of the Lie summand $a$ on its dual $a^{\prime}$ :

$$
\left(\theta^{a^{\prime}}(x) f\right)(y)=-\left(\theta(x)^{t} f\right)(y)
$$

for $x, y \in a, f \in a^{\prime}$, where $\theta$ is the adjoint representation of $g$ on $g$. The inner product on $g$ given by $(2.2 .1)$ is such that (2.3.2) holds:

$$
\theta(x)^{*}=\theta(\bar{x})
$$

for all $x$ in $g$. In the present situation the $g$ module $V_{\nu}$ is $g$ itself of course with $\nu=\theta$.

We know that $\eta$ is a unitary map of $a^{\prime}$ onto $\bar{a}_{\text {; }}$ see (2.3.5). Hence we can consider $\theta^{a^{\prime}}$ as a representation $\eta \theta^{a^{\prime}} \eta^{-1}$ of $a$ on $\bar{a}$. Let $P=P^{\bar{a}}$ be the orthogonal projection of $g$ onto $\bar{a}$.

Proposition 3.3.4 $\eta \theta^{a^{\prime}}(x) \eta^{-1}=\left.P^{\bar{a}} \theta(x)\right|_{\bar{a}}$ for all $x$ in $a$. 
Proof. Let $x, y, z \in a$ be arbitrary. Then by (2.3.3), (2.3.4), and (3.3.1)

$$
\begin{aligned}
\left(\theta^{a}(x) \eta^{-1}(\bar{y})\right)(z) & =-\left(\theta(x)^{t} \eta^{-1}(\bar{y})\right)(z)=-\eta^{-1}(\bar{y})(\theta(x) z) \\
& =-(\theta(x) z, \bar{y})=-([x, z], \bar{y})=(z,[x, \bar{y}]),
\end{aligned}
$$

by the invariance of the Killing form

$$
=\{[x, \bar{y}], \bar{z}\}=\{[x, \bar{y}], P \bar{z}\},
$$

by (2.2.1), (2.2.2),

$$
=\{P[x, \bar{y}], \bar{z}\}=(P[x, \bar{y}], z)=\left(\eta^{-1} \dot{P} \theta(x) \bar{y}\right)(z)
$$

which implies that $\theta^{\mathfrak{a}^{\prime}}(x) \eta^{-1}=\left.\eta^{-1} P \theta(x)\right|_{\bar{a}}$.

From now on then we shall consider $\theta^{a^{\prime}}$ as the representation of $a$ on $\bar{a}$ given by Proposition 3.3.4; i.e.

$$
\theta^{a \prime}(x) \bar{y}=P[x, \bar{y}]=P \theta(x) \bar{y}
$$

for $x, y \in a$. By Proposition 2.5.7 the coboundary operator $d_{\theta^{a^{\prime}}}$ on $\Lambda a^{\prime} \otimes \bar{a}$ for the representation $\theta^{a^{\prime}}$ is given by

$$
d_{\theta^{a^{\prime}}}=\sum_{i=1}^{m} \epsilon\left(z_{i}^{\prime}\right) \otimes \theta^{a^{\prime}}\left(z_{i}\right)+d \otimes 1=\sum_{i=1}^{m} \epsilon\left(z_{i}^{\prime}\right) \otimes P \theta\left(z_{i}\right)+d \otimes 1 ;
$$

i.e.

$$
d_{\theta^{a^{\prime}}}=\left.(1 \otimes P) d_{\left.\theta\right|_{a}}\right|_{\Lambda a^{\prime} \otimes \mathbf{a}}
$$

where $\left.\theta\right|_{a}$ is, of course, the adjoint representation of $a$ on 9. By Proposition 3.1 .3

$$
\left(d_{\theta^{a^{\prime}}}\right)^{*}=\left.(1 \otimes P)\left(d_{\left.\theta\right|_{a^{\prime}}}\right)^{*}\right|_{\Lambda a^{\prime} \otimes a}
$$

But

$$
d_{\theta \mid a}=\sum_{i=1}^{m} \epsilon\left(z_{i}^{\prime}\right) \otimes \theta\left(z_{i}\right)+d \otimes 1
$$

implies

$$
\left(d_{\left.\theta\right|_{a}}\right)^{*}=\sum_{i=1}^{m} \epsilon\left(z_{i}^{\prime}\right)^{*} \otimes \theta\left(\bar{z}_{i}\right)+d^{*} \otimes 1,
$$

b (3.3.2). Hence $\left(d_{\theta \mid a}\right)^{*}$ leaves $\Lambda a^{\prime} \otimes \bar{a}$ invariant ( $\bar{a}$ is a Lie subalgebra of $g$ by (2.2.4)). Then $(3.3 .8)$ implies

$$
\left(d_{\theta^{\prime \prime}}\right)^{*}=\left.\left(d_{\left.\theta\right|_{\mathbf{a}}}\right)^{*}\right|_{\Lambda \mathbf{a}^{\prime} \otimes \overrightarrow{\mathrm{a}}} \text {. }
$$

(3.3.7) and (3.3.9) imply that on $\Lambda a^{\prime} \otimes \bar{a}$ 


$$
\begin{aligned}
L_{\theta^{a^{\prime}}} & =d_{\theta^{a^{\prime}}}\left(d_{\theta^{a}}\right)^{*}+\left(d_{\theta^{a^{\prime}}}\right)^{*} d_{\theta^{a^{\prime}}} \\
& =(1 \otimes P) d_{\left.\theta\right|_{a}}\left(d_{\left.\theta\right|_{a}}\right)^{*}+\left(d_{\left.\theta\right|_{a}}\right)^{*}(1 \otimes P) d_{\left.\theta\right|_{a}} .
\end{aligned}
$$

Equation (3.3.10) is formally the same as equation (3.2.8). Therefore by arguments entirely similar to those which led to Theorem 3.2.12 we have

Theorem 3.3.11 Suppose a is a Lie summand of g. Let $\theta$ be the adjoint representation of $g$ on $g$ and let $\theta^{a^{\prime}}$ be the co-adjoint representation of $a$ on its dual $a^{\prime}$. Then the Laplacian $L_{\theta^{a^{\prime}}}$ corresponding to the representation $\theta^{a^{\prime}}$ is given by

$$
(\eta \otimes 1) L_{\theta^{\prime}}(\eta \otimes 1)^{-1}=(1 \otimes P) L_{\left.\theta\right|_{a}}(\eta \otimes 1)^{-1}+E
$$

on $\Lambda \bar{a} \otimes \bar{a}$ where $(\eta \otimes 1) L_{\left.\theta\right|_{a}}(\eta \otimes 1)^{-1}$ (the Laplacian for the adjoint representation of a on $g$ ) is given by Theorem 2.4 .7 (with $\nu=\theta$ ),

$$
\begin{aligned}
E= & \sum_{j=1}^{m} \sum_{i=1}^{m} \epsilon\left(\bar{z}_{j}\right) \iota\left(z_{i}\right) \otimes P^{\bar{a}} \theta\left(\bar{z}_{i}\right)\left(1-P^{\bar{a}}\right) \theta\left(z_{j}\right) \\
& +1 \otimes \sum_{j=1}^{m} P^{\bar{a}} \theta\left(\bar{z}_{j}\right)\left(P^{\bar{a}}-1\right) \theta\left(z_{j}\right),
\end{aligned}
$$

$\left\{z_{1}, \cdots, z_{m}\right\}$ is an orthonormal basis of $a$, and $P^{\bar{a}}$ is the orthogonal projection of $g$ onto $a$.

4. Restriction of the Laplacian to a weight space.

4.1 Choose a Cartan subalgebra $\mathfrak{G}$ of $g$ such that

$$
\mathfrak{G}=\overline{5}
$$

This is easy to do as we shall see later on. Now suppose that the Lie summand $a$ of $g$ and the $\nu(a)$ invariant subspace $U$ of $V_{\nu}$ satisfy the following additional invariance with respect to $b$ :

$$
\theta(5) a \subset a \text { and } \nu(5) U \subset U .
$$

The assumptions in (4.1.2) are actually rather mild ones and are satisfied immediately in most practical situations. Because of (4.1.1), (2.2.4), and (2.3.2) we deduce from (4.1.2) that $\bar{a}$ and $U^{\perp}$ are also invariant under $\bar{b}$ :

$$
\theta(\xi) \bar{a} \subset \bar{a} \text { and } \nu(\xi) U^{\perp} \subset U^{\perp} \text {. }
$$

Also since $\nu(G)$ leaves $U$ and $U^{\perp}$ invariant $\nu(G)$ commutes with the orthogonal projections $P^{U}, P^{\perp}=1-P^{U}$ of $V_{\nu}$ onto $U, U^{\perp}$, respectively:

$$
\left[\nu(H), P^{U}\right]=\left[\nu(H), P^{U^{\perp}}\right]=0
$$

for all $H$ in 5 . 
Proposition 4.1.5 For every $x$ in $g, \theta \otimes \nu(x)$ commutes with $c_{\nu}^{*}$. For every $x$ in $\bar{\zeta}, \theta \otimes \nu(x) d_{a, \nu}=d_{a, \nu} \theta \otimes \nu(x)$ on $\Lambda \bar{a} \otimes v_{\nu}$.

Proof. The first assertion is proved on p. 345 of [6]. We indicate how the second assertion follows from the first. Suppose $x \in \mathfrak{K}$. Then in particular $\theta \otimes \nu(x) c_{\nu}^{*}=c_{\nu}^{*} \theta \otimes \nu(x)$ on $\Lambda \bar{a} \otimes V_{\nu}$. By (2.5.6) we get

$$
\cdot \theta \otimes \nu(x)(\eta \otimes 1)\left(d_{\left.\nu\right|_{a}}\right)^{*}(\eta \otimes 1)^{-1}=(\eta \otimes 1)\left(d_{\left.\nu\right|_{a}}\right)^{*}(\eta \otimes 1)^{-1} \theta \otimes \nu(x)
$$

on $\Lambda \bar{a} \otimes V_{\nu}$ since $\Lambda \bar{a} \otimes V_{\nu}$ is $\theta \otimes \nu(x)$ invariant for $x$ in $\mathfrak{y}$; see (2.2.9) and (4.1.3). Taking the adjoint of both sides, using Proposition 3.1.3 and the fact that $\eta \otimes i$ is unitary, we get

$$
(\eta \otimes 1) d_{\left.\nu\right|_{a}}(\eta \otimes 1)^{-1} P^{\Lambda \bar{a} \otimes V \nu} \theta \otimes \nu(\bar{x})=P^{\Lambda \bar{a} \otimes V \nu} \theta \otimes \nu(\bar{x})(\eta \otimes 1) d_{\left.\nu\right|_{a}}(\eta \otimes 1)^{-1}
$$

on $\Lambda \bar{a} \otimes V_{\nu}$ where $P^{\Lambda \bar{a} \otimes V} \nu$ is the orthogonal projection of $\Lambda g \otimes V_{\nu}$ onto $\Lambda \bar{a} \otimes$ $V_{\nu}$; see (2.3.2) and (3.3.2). But $\Lambda \bar{a} \otimes V_{\nu}$ is also $\theta \otimes \nu(\bar{x})$ invariant since $\bar{x} \in \bar{G}$ by (4.1.1). Therefore we can drop the $P^{\Lambda \bar{a} \otimes V} \nu$ in the last equation. Applying (2.5.5) we obtain the desired results.

By (4.1.2) and (4.1.3) we can construct three representations $\beta^{(1)}, \beta^{(2)}, \beta^{(3)}$ of $\mathfrak{G}$ on $\Lambda \bar{a} \otimes U, \Lambda \bar{a} \otimes U^{\perp}, \Lambda \bar{a} \otimes \bar{a}$ respectively. We define

$$
\begin{aligned}
& \beta^{(1)}(H)=\left.\theta \otimes \nu(H)\right|_{\Lambda \bar{a} \otimes U}, \\
& \beta^{(2)}(H)=\left.\theta \otimes \nu(H)\right|_{\Lambda \bar{a} \otimes U}{ }^{\perp}, \\
& \beta^{(3)}(H)=\left.\theta \otimes \theta(H)\right|_{\Lambda \bar{a} \otimes \bar{a}},
\end{aligned}
$$

for $H$ in 5 .

Corollary 4.1.7 If a, $U$ satisfy (4.1.2), then $\beta^{(1)}$ commutes with $L_{\nu^{a}}, \beta^{(2)}$ commutes with $L_{\nu^{a^{\prime}}}$, and $\beta^{(3)}$ commutes with $L_{\theta^{a^{\prime}}}$ (see Theorems 3.1.12, 3.2.12, 3.3.11).

Proof. By (2.4.5), (2.5.5), and (2.5.6) we have

$$
L_{\left.\nu\right|_{\mathrm{a}}}=d_{\mathrm{a}, \nu} c_{\nu}^{*}+c_{\nu}^{*} d_{\mathrm{a}, \nu}
$$

on $\Lambda \bar{a} \otimes V_{\nu} \cdot$ By (3.1.9)

$$
L_{\nu a}=\left(1 \otimes P^{U}\right) L_{\left.\nu\right|_{a}}+E
$$

on $\Lambda \bar{a} \otimes U$ where $E=\left(1 \otimes P^{U}\right) d_{a, \nu}\left[1 \otimes P^{U}, c_{\nu}^{*}\right]$. Therefore $\beta^{(1)}$ commutes with $E$ and $L_{\left.\nu\right|_{a}}$ on $\Lambda \bar{a} \otimes U$ by (4.1.4) and Proposition 4.1.5. So $\beta^{(1)}$ commutes with $L_{\nu^{a^{*}}}$ The second and third assertions of Corollary 4.1 .7 follow similarly。 
4.2 Let $\xi_{0}$ be a maximal abelian subalgebra of the compact real form $E$ which was chosen in 2.2 . Then

$$
\xi=\epsilon_{0}+\sqrt{-1} \epsilon_{0}
$$

is a Cartan subalgebra of $g$ such that

$$
\pi 5=5
$$

where $r$ is conjugation of $g$ with respect to $t$. Let $\Delta$ denote the set of nonzero roots of $g$ with respect to $\mathscr{G}$ and let $\mathfrak{G}_{R}$ be the real vector space spanned by the $H_{a} \in \mathcal{G}, a \in \Delta$, where

$$
\left(H, H_{a}\right)=\alpha(H)
$$

for all $H$ in $\mathfrak{G}_{0}$. The Killing form is positive definite on $\mathfrak{G}_{\mathrm{R}}$. By definition $\bar{x}=$ $-\tau x, x \in 9$; see (2.2.2). Hence one can deduce from (4.2.2) the equations

$$
\bar{G}=\bar{g} \text { and } \bar{g}_{a}=g_{-a}
$$

where $g_{a}$ is the root space corresponding to $a \in \Delta$; cf. (4.1.1). Moreover $\bar{H}=H$ for all $H$ in $h_{R}$. Relative to some lexicographic ordering on $\mathscr{G}_{R}^{\prime}$ let $\Delta^{+}$be a choice of positive roots and let $\pi$ be a corresponding system of simple roots. Define

$$
n=\sum_{a \in \Delta^{+}} 9 a
$$

$\mathfrak{n}$ is a nilpotent subalgebra of $\mathrm{g}$ and, in fact, $\mathfrak{n}$ is the nilradical of the Borel subalgebra

$$
b=5+n
$$

of g. By (4.1.4)

$$
\overline{\mathfrak{n}}=\sum_{a \in \Delta^{+}} g_{-a}
$$

and since $(x, y)=0$ for $x \in g_{\alpha}, y \in g_{\beta}$ with $a \neq-\beta$ it follows that

$$
g=n+\bar{b}+\bar{n}
$$

is an orthogonal direct sum decomposition of $g_{0}$ Moreover

$$
n^{\perp}=\bar{g}+\bar{n}
$$

so that $n$ is a Lie summand of $g$.

For each $\alpha$ in $\Delta$ we choose, once and for all, $e_{a}$ in $g_{a}$ such that

$$
\left(e_{a}, \bar{e}_{a}\right)=1 \text {. }
$$

If $\left\{H_{1}, \cdots, H_{l}\right\}$ is any orthonormal basis of $\bar{G}_{\text {, then }}\left\{H_{1}, \cdots, H_{l}\right\} \cup\left\{\bar{e}_{a}\right\}{ }_{a \in \Delta^{+}}$is an orthonormal basis of $n^{\perp}$ and $\left\{e_{a}\right\}$ is an orthonormal basis of $n_{\text {. }}$ 
The Weyl group of $g$ relative to $\mathscr{G}$ will be denoted by $W . W$ is generated, in fact, by the simple Weyl reflections $S_{a}, \alpha \in \pi$, where $S_{a}: G_{R}^{\prime} \rightarrow G_{R}^{\prime}$ is defined by

$$
s_{\alpha} \xi=\xi-2(\xi, \alpha) \alpha /(\alpha, \alpha), \quad \xi \in \xi_{R}^{\prime} .
$$

As usual we define

$$
\delta=\frac{1}{2} \sum_{\beta \in \Delta^{?}} \beta
$$

We shall make use of the following well-known (or not so well-known) proposition. For a proof see Corollary 8.3.8.

Proposition 4.2.13 Let $\beta \in \Delta^{+}$. Then $\beta \in \pi$ if and only if $(2 \delta, \beta)=(\beta, \beta)$.

4.3 Since $\nu$ is finite dimensional $\nu$ has a highest weight $\lambda \in \mathfrak{G}^{\prime}$ :

$$
\nu(n) V_{\nu}^{\lambda}=\{0\}
$$

where

$$
V_{\nu}^{\lambda}=\left\{v \in V_{\nu} \mid \nu(H) v=\lambda(H) v \text { for all } H \text { in } 5\right\}
$$

is the weight space of $\nu$ corresponding to $\lambda$. The Casimir operator

$$
C^{\nu}=\sum_{i=1}^{n} \nu\left(\bar{z}_{i}\right) \nu\left(z_{i}\right)
$$

where, as earlier, $\left\{z_{1}, \cdots, z_{m}\right\},\left\{z_{m+1}, \cdots, z_{n}\right\}$ are orthonormal bases for $a, a^{\perp}$, respectively, is a scalar on $V_{\nu}^{\lambda}$. The scalar is given by

$$
\left.C^{\nu}\right|_{\nu} ^{\lambda}=(\lambda, \lambda+2 \delta) 1_{V_{\nu}^{\lambda}}
$$

see [5]. In particular when $\nu$ is irreducible

$$
C^{\nu} v=(\lambda, \lambda+2 \delta) v
$$

for all $v$ in $V_{\nu}$.

From now on we shall consider the important case when the Lie summand a is taken to be the nilradical $n$ of $b$; see (4.2.5), (4.2.6). Clearly $n$ satisfies (4.1.2). There is a representation $\beta^{(0)}$ of $\bar{G}$ on $\Lambda \bar{n} \otimes V_{\nu}$ defined by

$$
\beta^{(0)}(H)=\left.\theta \otimes \nu(H)\right|_{\Lambda \bar{\pi} \otimes V \nu}
$$

for $H$ in $\mathscr{G}$; cf. (4.1.6). Given $\xi \in \mathfrak{G}^{\prime}$ set

$$
\left(\Lambda \bar{n} \otimes V_{\nu}\right)^{\xi}=\left\{f \in \Lambda \bar{n} \otimes V_{\nu} \mid \beta^{(0)}(H) f=\xi(H) f \forall H \in \zeta\right\} .
$$

As expected we say that $\xi$ is a weight of $\beta^{(0)}$ if $\left(\Lambda \bar{n} \otimes V_{\nu}\right)^{\xi} \neq\{0\}$. In this case the multiplicity of $\xi$ is the dimension of the weight space $\left(\Lambda \bar{n} \otimes V_{\nu}\right)^{\xi}$. This terminology extends of course to any representation of $\mathfrak{b}$. 
Theorem 4.3.8 If $\nu$ is irreducible, then the Laplacian $L_{\left.\nu\right|_{\mathfrak{n}}}$ acts on $\left(\Lambda \bar{n} \otimes V_{\nu}\right)^{\xi}$ as a scalar, for any $\xi$ in $\xi^{\prime}$. The value of the scalar is $1 / 2[(\lambda+\delta, \lambda+\delta)-(\xi+\delta, \xi+\delta)]$ where $\lambda$ is the highest weight of $\nu$ and $\delta$ is given by (4.2.12).

Before proceeding to the proof we remark that Theorem 4.3.8 is essentially a version of Theorem 5.7 of [6] which gives the spectral resolution of the Laplacian. We allow $\xi$ to be an arbitrary (complex) linear functional on $\xi$.

Proof. Let $\left\{H_{i}\right\}_{i=1}^{l}$ be any orthonormal basis of $\mathscr{h}$. Then as we have observed earlier $\left\{H_{i}\right\}_{i=1}^{l} \cup\left\{\bar{e}_{\beta}\right\}_{\beta \in \Delta^{+}}$is an orthonormal basis of $n^{\perp}$ and $\left\{e_{\beta \in \Delta^{+}}\right.$is an orthonormal basis of $n$. By Theorem 2.4.7

$$
\begin{aligned}
2 L_{\left.\nu\right|_{\mathfrak{n}}=} & 1 \otimes C^{\nu}+\sum_{\beta \in \Delta^{+}} \theta \otimes \nu\left(\bar{e}_{\beta}\right) \theta \otimes \nu\left(e_{\beta}\right) \\
& -\sum_{i=1}^{l} \theta \otimes \nu\left(\bar{H}_{i}\right) \theta \otimes \nu\left(H_{i}\right)-\sum_{\beta \in \Delta^{+}} \theta \otimes \nu\left(e_{\beta}\right) \theta \otimes \nu\left(\bar{e}_{\beta}\right)
\end{aligned}
$$

on $\Lambda \bar{n} \otimes V_{\nu}$. Since for each $\beta$ in $\Delta^{+},\left(e_{\beta}, \bar{e}_{\beta}\right)=1$ we have

$$
\left[e_{\beta}, \bar{e}_{\beta}\right]=H_{\beta} \quad(\operatorname{see}(4.2 .3)) \text {. }
$$

Therefore

$$
\begin{aligned}
\theta \otimes \nu\left(\vec{e}_{\beta}\right) \theta \otimes \nu\left(e_{\beta}\right) & =\theta \otimes \nu\left(e_{\beta}\right) \theta \otimes \nu\left(\bar{e}_{\beta}\right)+\left[\theta \otimes \nu\left(\bar{e}_{\beta}\right), \theta \otimes \nu\left(e_{\beta}\right)\right] \\
& =\theta \otimes \nu\left(e_{\beta}\right) \theta \otimes \nu\left(\bar{e}_{\beta}\right)-\theta \otimes \nu\left(H_{\beta}\right)
\end{aligned}
$$

implies

$$
\begin{aligned}
2 L_{\left.\nu\right|_{\mathfrak{n}}} & =1 \otimes C^{\nu}-\sum_{i=1}^{l} \theta \otimes \nu\left(\bar{H}_{i}\right) \theta \otimes \nu\left(H_{i}\right)-\sum_{\beta \in \Delta^{+}} \theta \otimes \nu\left(H_{\beta}\right) \\
& =1 \otimes C^{\nu}-\sum_{i=1}^{l} \beta^{(0)}\left(\bar{H}_{i}\right) \beta^{(0)}\left(H_{i}\right)-\sum_{\beta \in \Delta^{+}} \beta^{(0)}\left(H_{\beta}\right)
\end{aligned}
$$

on $\Lambda \bar{n} \otimes V_{\nu}$ by (4.3.6). Also since $\left\{H_{i}\right\}_{i=1}^{l}$ and $\left\{\bar{H}_{i}\right\}_{i=1}^{l}$ are orthonormal bases for $\bar{G}=\bar{G}$ one easily checks that

$$
(H, H)=\sum_{i=1}^{l}\left(H, \bar{H}_{i}\right)\left(H, H_{i}\right)
$$

for all $H$ in 5 . It follows that for an arbitrary $f$ in $\left(\Lambda \bar{n} \otimes V_{\nu}\right)^{\xi}$ (see (4.3.7)) 


$$
\begin{aligned}
\sum_{i=1}^{l} \beta^{(0)}\left(\bar{H}_{i}\right) \beta^{(0)}\left(H_{i}\right) f & =\sum_{i=1}^{l} \xi\left(\bar{H}_{i}\right) \xi\left(H_{i}\right) f=\sum_{i=1}^{l}\left(H_{\xi}, \bar{H}_{i}\right)\left(H_{\xi}, H_{i}\right) f \\
& =\left(H_{\xi}, H_{\xi}\right) f=(\xi, \xi) f .
\end{aligned}
$$

Also

$$
\sum_{\beta \in \Delta^{+}} \beta^{(0)}\left(H_{\beta}\right) f=\sum_{\beta \in \Delta^{+}} \xi\left(H_{\beta}\right) f=\left(\xi, \sum_{\beta \in \Delta^{+}} \beta\right) f=(\xi, 2 \delta) f .
$$

Therefore by (4.3.5)

$$
2 L_{\left.\nu\right|_{\mathfrak{n}}} f=[(\lambda, \lambda+2 \delta)-(\xi, \xi)-(\xi, 2 \delta)] f=[(\lambda+\delta, \lambda+\delta)-(\xi+\delta, \xi+\delta)] f
$$

which proves the the orem.

One proves $(4.3 .4)$ by arguments very similar to those just given as a matter of fact.

From Corollary 4.1.7 we deduce that the Laplacians $L_{\nu^{n}}, L_{\nu^{n^{n}}}, L_{\theta^{n^{\prime}}}$ leave weight spaces of the representations $\beta^{(1)}, \beta^{(2)}, \beta^{(3)}($ see $(4.1 .6)$ ) invariant. However these Laplacians do not reduce to a scalar on a weight space because of the complex nature of the error terms $E$.

5. Determination of the cohomology group $H^{1}(n, n)$.

5.1 From this point on we shall assume that the representation $\nu$ is the adjoint representation $\theta$ of $g$ on $g$ and that $\theta$ is irreducible; i.e. $g$ is simple. Thus $V_{\nu}=$ g. The highest weight (highest root) of $\theta$ will be denoted by $\lambda$. Under the adjoint action $n$ is an $n$ module so one can inquire about the cohomology groups $H^{j}(n, n), j \geq 0$; see 2.4. In particular what is the structure of $H^{1}(n, n)$ as a vector space over $C$ ?

Professor Kostant's results in [6] cannot be applied to answer this question directly because $n$ is not a $g$ module. However he has computed $H^{1}(n, n)$ by other means in an unpublished manuscript. We shall obtain his results, independently, by the application of Theorem 3.1.12 with $a=n, U=n$.

In principal, but not necessarily in practice, the Hodge decomposition permits the determination of cohomology in any degree. For example

$$
H^{j}(n, n)=\left\{x \in \Lambda^{j} \bar{n} \otimes n \mid L_{\theta^{n}} x=0\right\}
$$

where $L_{\theta^{n}}$ is given by Theorem 3.1.12. The problem is reduced therefore to that of finding the zeroes of various Laplace operators.

By (2.4.3) and (2.4.1) with $k=1$ one sees at the outset that

$$
H^{1}(n, n)=\begin{gathered}
\text { all derivations of } n \\
\text { inner derivations of }-n
\end{gathered}
$$

Thus, in particular, we shall obtain the full automorphism group of $n$. 
By (4.2.8) and (4.2.9)

$$
g=n+\bar{b}+\bar{n}, \quad \bar{g}+\bar{n} \cdot=n^{+},
$$

is an orthogonal direct sum decomposition of $g$. Any $z$ in $g$ is written uniquely $z=x+H+\bar{y}$ where $x, y \in \mathfrak{n}, H \in \mathfrak{h}$. Let $P$ be the orthogonal projection of $g$ onto $n$. Hence $P z=x$. By $(4,1,4) P$ commutes with $\theta(5)$; of course this is trivial to check directly.

The Laplacian $L_{\theta^{n}}$ which corresponds to the adjoint representation $\theta^{n}$ of $n$ on $n$ is given by Theorem 3.1.12:

$$
L_{\theta^{\mathfrak{n}}}=(1 \otimes P) L_{\left.\theta\right|_{\mathfrak{n}}}+E
$$

on $\Lambda \bar{n} \otimes n$ where

$$
E=\sum_{\beta \in \Delta^{+}} \sum_{\gamma \in \Delta^{+}}\left(\bar{e}_{\beta}\right) \iota\left(e_{\gamma}\right) \otimes P \theta\left(e_{\beta}\right)(P-1) \theta\left(\bar{e}_{\gamma}\right) .
$$

Let $\beta^{(1)}$ be the representation of $\bar{G}$ on $\Lambda \bar{n} \otimes n$ given by (4.1.6). The vectors $\left\{\bar{e}_{\beta} \otimes e_{\gamma}\right\}_{\gamma, \beta \epsilon \Delta^{+}}$form a basis of $\Lambda^{1} \bar{n} \otimes n$. Moreover for every $H$ in $\mathfrak{G}$

$$
\begin{aligned}
\beta^{(1)}(H) \bar{e}_{\beta} \otimes e_{\gamma} & =\theta \otimes \theta(H) \bar{e}_{\beta} \otimes e_{\gamma}=\left[H, \bar{e}_{\beta}\right] \otimes e_{\gamma}+\bar{e}_{\beta} \otimes\left[H, e_{\gamma}\right] \\
& =(\gamma-\beta)(H) \bar{e}_{\beta} \otimes e_{\gamma} .
\end{aligned}
$$

This means that the linear forms $\gamma-\beta, \gamma, \beta \in \Delta^{+}$are weights of $\beta^{(1)}$ and they represent all weights of $\left.\beta^{(1)}\right|_{\Lambda^{1} \bar{n} \otimes n^{\circ}}$ We can add that

$$
\Lambda^{1} \bar{n} \otimes n=\sum_{\xi} \bigoplus\left(\Lambda^{1} \bar{n} \otimes n\right)^{\xi}
$$

where $\xi$ varies over the distinct weights $\gamma-\beta ; \gamma, \beta \in \Delta^{+}$, and if $x \in \Lambda^{1} \bar{n} \otimes n$ then $x \in\left(\Lambda^{1} \bar{n} \otimes n\right)^{\xi}$ if and only if

$$
x=\sum_{\gamma, \beta \epsilon \Delta^{+} ; \gamma-\beta=\xi} c_{\beta \gamma} \bar{e}_{\beta} \otimes e_{\gamma}, \quad c_{\beta \gamma} \in \mathbf{C} .
$$

These observations follow immediately.

We define

$$
\left(\Lambda^{1} \overline{\mathfrak{n}} \otimes n\right)_{0}^{\xi}=\left\{x \in\left(\Lambda^{1} \bar{n} \otimes n\right)^{\xi} \mid L_{\theta^{n}} x=0\right\}
$$

for $\xi \in \mathscr{G}^{\prime}$. Because the weight spaces $\left(\Lambda^{1} \bar{n} \otimes n\right)^{\xi}$ are $L_{\theta^{n}}$ invariant $\left(L_{\theta^{n}}\right.$ and $\beta^{(1)}$ commute) (5.1.1) and (5.1.5) imply

$$
\text { (5.1.8) } H^{1}(n, n)=\sum_{\text {distinct weights } \xi=\gamma-\beta ; \gamma, \beta \in \Delta^{+}}\left(\Lambda^{1} \bar{n} \otimes n\right)_{0}^{\xi} \quad \text { (direct sum). }
$$

5.2 Lemma 5.2.1 For $\mu, \nu \in \Delta^{+}, E \bar{e}_{\mu} \otimes e_{\nu}=0$ unless $\nu-\mu \in\{0\} \cup \Delta^{-}$ 
(where $\Delta^{-}$is the set of negative roots). If $\nu-\mu \in\{0\} \cup \Delta^{-}$then

$$
E \bar{e}_{\mu} \otimes e_{\nu}=\sum_{\beta \epsilon \Delta^{+} ; \beta+\nu-\mu \epsilon \Delta^{+}} \bar{e}_{\beta} \otimes\left[e_{\beta},\left[\bar{e}_{\mu}, e_{\nu}\right]\right] .
$$

Proof. By (2.2.8), $\iota\left(e_{\gamma}\right) \bar{e}_{\mu}=\left(e_{\gamma}, \bar{e}_{\mu}\right)=\delta_{\gamma \mu}$. Therefore (5.1.3) and $(2.2 .7)$ imply that for any $\mu, \nu \in \Delta^{+}$

$$
\begin{aligned}
E \vec{e}_{\mu} \otimes e_{\nu} & =\sum_{\beta \in \Delta^{+}} \sum_{\gamma \in \Delta^{+}} \epsilon\left(\vec{e}_{\beta}\right) \iota\left(e_{\gamma}\right) \bar{e}_{\mu} \otimes P \theta\left(e_{\beta}\right)(P-1) \theta\left(\bar{e}_{\gamma}\right) e_{\nu} \\
& =\sum_{\beta \in \Delta^{+}} \bar{e}_{\beta} \otimes P \theta\left(e_{\beta}\right)(P-1) \theta\left(\bar{e}_{\mu}\right) e_{\nu^{\cdot}}
\end{aligned}
$$

If $\nu-\mu$ is not a root then $\theta\left(\bar{e}_{\mu}\right) e_{\nu}=\left[\bar{e}_{\mu}, e_{\nu}\right]=0 \rightarrow E \bar{e}_{\mu} \otimes e_{\nu}=0$. Assume $\nu-\mu$ is a root. If $\nu-\mu \in \Delta^{+}$then (by definition of $P$ )

$$
(P-1) \theta\left(\bar{e}_{\mu}\right) e_{\nu}=0 \rightarrow E \bar{e}_{\mu} \otimes e_{\nu}=0 .
$$

If $\nu-\mu \in\{0\} \cup \Delta^{-}$(where $\Delta^{-}$is the set of negative roots) then $\theta\left(\bar{e}_{\mu}\right) e_{\nu} \in n^{\perp} \rightarrow$ $(P-1) \theta\left(\bar{e}_{\mu}\right) e_{\nu}=\theta\left(\bar{e}_{\mu}\right) e_{\nu}$ so that

$$
E \bar{e}_{\mu} \otimes e_{\nu}=\sum_{\beta \in \Delta^{+}} \bar{e}_{\beta} \otimes P\left[e_{\beta},\left[\bar{e}_{\mu}, e_{\nu}\right]\right]
$$

By arguments similar to those just given the only terms which survive in the last sum are those for which $\beta-\mu+\nu \in \Delta^{+}$. The proof is therefore complete.

Proposition 5.2.2 Let $\nu$ be a finite dimensional representation of 9 with highest weight $\lambda$. Then $|\lambda+\delta|>|\xi+\delta|$ for any weight $\xi$ of $\nu$ such that $\xi \neq \lambda$.

For a proof see [5, p. 248].

Corollary 5.2.3 If $\mu \in \Delta^{+}$and $\nu$ is a root such that $\nu-\mu$ is a root then $|\lambda+\delta|>|\delta+\nu-\mu|$.

Proof. Since $\lambda$ is the highest root we cannot have $\lambda=\nu-\mu$ for $\mu \in \Delta^{+}$.

The proof of the next lemma, up to a point, is essentially Cartier's simplified proof of Lemma 5.12 in [6]; see [1].

Lemma 5.2.4 Let $(\mu, \nu) \in \Delta^{+} \times \Delta$. Then $|\lambda+\delta| \geq|\delta+\nu-\mu|$ with equality if and only if $\mu \in \pi$ and $\nu=S_{\mu} \lambda$ where $S_{\mu}$ is the simple Weyl reflection defined by $\mu$ (see 4.2.11). Moreover if $\lambda \notin \pi$ the weight $S_{\mu} \lambda-\mu, \mu \in \pi$, bas multiplicity one.

Proof. If $\mu \in \pi$ then $S_{\mu} \delta=\delta-\mu$ (see [5, p. 248]) so clearly $\nu=S_{\mu} \lambda \rightarrow$ $|\delta+\nu-\mu|=\left|S_{\mu}(\lambda+\delta)\right|=|\lambda+\delta|$. Given $\psi \subset \Delta$ put

$$
\Phi=\Phi_{\psi}=\psi \cap \Delta^{+} \subset \Delta^{+}
$$


If $\Phi \subset \Delta^{+}$put

$$
\psi=\psi_{\Phi}=\Phi \cup-\left\{\Delta^{+} \cap(\Delta \sim \Phi)\right\}
$$

where $\Delta \sim \Phi$ is the complement of $\Phi$ in $\Delta$. The mapping $\Phi \rightarrow \psi_{\Phi}$ is a 1.1 mapping of the family of subsets $\Phi$ of $\Delta^{+}$onto the family of subsets $\psi$ of $\Delta$ such that $\Delta=\psi \cup-\psi$ is a disjoint union. Moreover if $\Phi \subset \Delta^{+}$then

$$
\delta-\langle\Phi\rangle=-1 / 2\left\langle\psi_{\phi}\right\rangle
$$

where we define

$$
\langle A\rangle=\sum_{\beta \in A} \beta \text { for } A \subset \Delta .
$$

In particular for $\Phi=\{\mu\}$ a single positive root $\mu$ (5.2.7) simply says that

$$
\delta-\mu=-\frac{1}{2}\left(\mu-\sum_{\beta \in \Delta^{+} ; \beta \neq u} \beta\right) \text {. }
$$

Given $\mu \in \Delta^{+}$and $\nu \in \Delta$

$$
\delta+\nu-\mu=\nu-1 / 2\langle\psi\rangle
$$

where $\psi=\{\mu\} \cup-\left\{\beta \in \Delta^{+} \mid \beta \neq \mu\right\}$ by (5.2.8). If $S$ is an arbitrary element of the Weyl group $W$ then by (5.2.9)

$$
S(\delta+\nu-\mu)=S \nu-1 / 2\langle S \psi\rangle=\delta+S \nu-\delta-1 / 2\langle S \psi\rangle
$$

Since $\Delta=S \psi \cup-S \psi$ is a disjoint union

$$
S \psi=\psi_{\Phi}
$$

for a unique $\Phi_{S} \subset \Delta^{+}$. In fact $\Phi_{S}$ is given by

$$
\Phi_{S}=S \psi \cap \Delta^{+} \text {. }
$$

By (5.2.7) $\delta-\left\langle\Phi_{S}\right\rangle=-1 / 2\left\langle\psi_{\Phi_{S}}\right\rangle$ so (5.2.10) and (5.2.11) imply

$$
S(\delta+\nu-\mu)=\delta+S \nu-\left\langle\Phi_{S}\right\rangle
$$

Since $\lambda$ is the highest root and $S \nu \in \Delta, S \nu=\lambda-\Sigma_{\beta \in \Delta^{+}} n_{\beta} \beta$ where the $n_{\beta}$ are nonnegative integers. Then (5.2.13) implies

$$
s(\delta+\nu-\mu)=\delta+\lambda-\sum_{\beta \in \Delta^{+}} n_{\beta}^{\prime} \beta
$$

where the $n_{\beta}^{\prime}$ are nonnegative integers. In particular we choose $S \in W$ such that (5.2.15)

$$
(S(\delta+\nu-\mu), \beta) \geq 0 \quad \forall \beta \in \Delta^{+}
$$

(this can be done since $\delta+\nu-\mu \in \mathfrak{G}_{\mathrm{R}}$ ). Hence by (5.2.14) 


$$
|\lambda+\delta|^{2}=|\delta+\nu-\mu|^{2}+2 \sum_{\beta \in \Delta^{+}} n_{\beta}^{\prime}(S(\delta+\nu-\mu), \beta)+\left|\sum_{\beta \in \Delta^{+}} n_{\beta}^{\prime} \beta\right|^{2} \geq|\delta+\nu-\mu|^{2},
$$

which was to be shown. Now suppose $|\lambda+\delta|=|\delta+\nu-\mu|$. Then each $n_{\beta}^{\prime}$ must be zero so that

$$
S(\delta+\nu-\mu)=\lambda+\delta
$$

by (5.2.14). By (5.2.13) and (5.2.16) $\lambda+\delta=\delta+S \nu-\left\langle\Phi_{S}\right\rangle$ or $\lambda=S \nu-\left\langle\Phi_{S}\right\rangle=$ $\lambda-\Sigma_{\beta \in \Delta^{+}{ }_{\beta} \beta} \beta-\left\langle\Phi_{S}\right\rangle$.

Consequently each $n_{\beta}=0$ and $\Phi_{S}=\varnothing$. But then $S \nu=\lambda$ and (5.2.12) which implies

$S \psi \cap \Delta^{+}=\varnothing$ which implies $\psi_{\Phi}=S^{-1} \Delta^{-}$

so that $\Phi=\psi_{\Phi} \cap \Delta^{+}=S^{-1} \Delta^{-} \cap \Delta^{+}$. On the other hand $\Phi=\{\mu\}$. This says that the length of $S$ is one; see [4]. Thus we must have $S=S_{a}$, some $a \epsilon \pi$. By $(5.2 .16)$

$$
\lambda+\delta=S_{\alpha} \delta+\lambda-S_{\alpha} \mu=\delta-\alpha+\lambda-S_{\alpha} \mu \text { or } S_{\alpha} \mu=-\alpha=S_{\alpha} \alpha
$$

implies $\mu=\alpha$; i.e. $\mu \in \pi$ and $S_{\mu} \nu=\lambda$ or $\nu=S_{\mu} \lambda$ since $S_{\mu}^{2}=1$. Finally if $\lambda \neq \mu$, $\mu \in \pi$, then $S_{\mu} \lambda \in \Delta^{+}$. Let $x \in\left(\Lambda^{1} \bar{n} \otimes n\right)^{S_{\mu} \lambda-\mu}$. Then by (5.1.6)

$$
x=\sum_{\beta, \gamma \in \Delta^{+} ; \gamma-\beta=S_{\mu \lambda-\mu}} c_{\beta \gamma} \bar{e}_{\beta} \otimes e_{\gamma}, \quad c_{\beta \gamma} \in \mathbf{C} .
$$

But $\gamma-\beta=S_{\mu} \lambda-\mu$ implies (since $\mu \in \pi$ ) $\gamma-\beta+\delta=S_{\mu} \lambda-\mu+\delta=S_{\mu}(\lambda+\delta$ ) implies $|\delta+\gamma-\beta|=|\lambda+\delta|$ so as we have just seen

$$
\left.\gamma-\beta=S_{\beta} \lambda-\beta \quad \text { (with } \beta \epsilon \pi\right) \quad \text { or } \quad S_{\beta} \lambda-\beta=S_{\mu} \lambda-\mu \text {. }
$$

Therefore $\beta=\mu$ and $\gamma=S_{\mu} \lambda$ (by (4.2.11)) and $\operatorname{dim}\left(\Lambda^{1} \bar{n} \otimes n\right)^{S} \mu^{\lambda-\mu}=1$. Of course

$$
\left(\Lambda^{1} \bar{n} \otimes n\right)^{S_{\mu} \lambda-\mu}=\mathrm{C}\left(\bar{e}_{\mu} \otimes e_{S_{\mu} \lambda}\right) \text { for } \mu \in \pi, \lambda \neq \mu .
$$

5.3 If $\xi \in \mathfrak{h}^{\prime}$ then we shall put

$$
c(\xi)=1 / 2\left(|\lambda+\delta|^{2}-|\delta+\xi|^{2}\right) .
$$

From Theorem 4.3.8 and (5.1.3) we derive

$$
L_{\theta^{\mathfrak{n}}}=c(\xi) 1+E \quad \text { on }(\Lambda \bar{n} \otimes n)^{\xi} \text {. }
$$

Proposition 5.3.3 For each simple root $\mu$ such that $S_{\mu} \lambda \in \Delta^{+}$we have

$$
\left(\Lambda^{1} \overline{\mathfrak{n}} \otimes n\right)_{0}^{S_{\mu} \lambda-\mu}=\left(\Lambda^{1} \bar{n} \otimes n\right)^{S_{\mu \lambda-\mu}}=\mathrm{C}_{\bar{e}_{\mu}} \otimes e_{s_{\mu} \lambda} .
$$

Proof. By Lemma 5.2.4 $c\left(S_{\mu} \lambda-\mu\right)=0$. Therefore Corollary 5.2.3 implies that $S_{\mu} \lambda-\mu$ is not a root. By (5.1.6), Lemma 5.2.1, and (5.3.2) $L_{\theta^{n}}=0$ on 
$\left(\Lambda^{1} \bar{n} \otimes n\right)^{S_{\mu} \mu^{\lambda-\mu}}$. Proposition 5.3.3 now follows by (5.2.17).

It is useful to observe that for $x \in \Lambda \bar{\pi} \otimes n$

$$
L_{\theta^{\mathfrak{n}}} x=0 \text { if and only if } d_{\theta^{\mathfrak{n}}} x \text { and }\left(d_{\theta^{\mathfrak{n}}}\right)^{*} x=0 .
$$

Also for $x \in \Lambda^{1} \dot{\bar{n}} \otimes n$

$$
d_{\theta^{\mathfrak{n}^{x}}}=0
$$

if and only if $x$ is a derivation of $n$ where we identify $\Lambda^{1} \bar{n} \otimes n$ with $\Lambda^{1} n^{\prime} \otimes n$ (by (2.3.6)) and $\Lambda^{1} n^{\prime} \otimes n$ with the space $L(n, n)$ of linear operators on $n$. These remarks follow by (3.1.4), Proposition 2.1.3(i), and (2.4.1). With these identifications

$$
\bar{e}_{\beta} \otimes x: y \rightarrow f_{\beta}(y) x, \quad x, y \in n,
$$

where $\left\{f_{\beta}\right\}_{\beta \in \Delta^{+}}$is the basis of $n^{\prime}$ dual to $\left\{e_{\beta}\right\}_{\beta \in \Delta^{+}}$.

Proposition 5.3.7 Let $\gamma, \beta \in \Delta^{+}$such that $\gamma \neq \beta$ and $c(\gamma-\beta) \neq 0$. Then $\left(\Lambda^{1} \bar{n} \otimes n\right)_{0}^{\gamma-\beta}=\{0\}$.

Proof. Take

$$
x=\sum_{\mu, \nu \in \Delta^{+} ; \nu-\mu=\gamma-\beta} c_{\mu \nu} \bar{e}_{\mu} \otimes e_{\nu}, \quad \epsilon\left(\Lambda^{1} \bar{n} \otimes n\right)^{\gamma-\beta}, c_{\mu \nu} \in \mathrm{C} ;
$$

see (5.1.6). By (5.3.2) $L_{\theta^{n^{x}}} x=0$ if and only if $E x=-c(\gamma-\beta) x$. If $\gamma-\beta$ is not in $\Delta^{-}$then $E x=0$ by Lemma 5.2.1. Hence $L_{\theta^{n^{x}}}=0$ and $c(\gamma-\beta) \notin 0$ imply that $x=0$. Assume $\gamma-\beta \in \Delta^{-}$. By Lemma 5.2.1

$$
E x=\sum_{\mu, \nu \in \Delta^{+} ; \nu-\mu=\gamma-\beta} c_{\mu \nu} \sum_{a \in \Delta^{+} ; \alpha+\gamma-\beta \in \Delta^{+}} \bar{e}_{\alpha} \otimes\left[e_{\alpha},\left[\bar{e}_{\mu}, e_{\nu}\right]\right] .
$$

Clearly $\gamma-\beta \in \Delta^{-}, \alpha \in \Delta^{+}$and $\alpha+\gamma-\beta \epsilon \Delta^{+}$imply $\alpha \notin \pi$ so (5.3.6) implies $(E x)\left(e_{\xi}\right)=0 \quad \forall \xi \in \pi$. Hence if $L_{\theta^{\mathfrak{n}}} x=0$, then $c(\gamma-\beta) \neq 0$ implies $x=$ $(-1 / c(\gamma-\beta)) E x$ so that

$$
x\left(e_{\xi}\right)=0 \quad \forall \xi \in \pi .
$$

By (5.3.4), (5.3.5) $x$ is a derivation of $n$. Therefore $x=0$ by (5.3.8). Q.E.D.

Proposition 5.3.9 Identify $\Lambda^{1} \bar{n} \otimes n$ with the space $L(n, n)$ of linear operators on $n$; see (5.3.6). Let $x \in\left(\Lambda^{1} \bar{n} \otimes n\right)^{0}$. Then $L_{\theta^{n^{n}}} x=0$ if and only if $x=$ $\Sigma_{\beta \in \Delta^{+}}{ }^{c}{ }_{\beta} \theta\left(H_{\beta}\right), c_{\beta} \in \mathbf{C}$, where $H_{\beta}$ is given by (4.2.3).

Proof. $x=\Sigma_{\mu \epsilon \Delta^{+}} c_{\mu} \bar{e}_{\mu} \otimes e_{\mu}, c_{\mu} \in \mathrm{C}$, by (5.1.6). For each $\mu \in \Delta^{+}$

$$
E \bar{e}_{\mu} \otimes e_{\mu}=\sum_{\beta \in \Delta^{+}} \bar{e}_{\beta} \otimes\left[e_{\beta},\left[\bar{e}_{\mu}, e_{\mu}\right]\right]=\sum_{\beta \in \Delta^{+}} \bar{e}_{\beta} \otimes\left[H_{\mu}, e_{\beta}\right]
$$


by Lemma 5.2.1 and (4.3.9). Therefore for every $y$ in $n$ (5.3.6) implies

$$
\left(E \bar{e}_{\mu} \otimes e_{\mu}\right)(y)=\sum_{\beta \in \Delta^{+}} f_{\beta}(y)\left[H_{\mu}, e_{\beta}\right]=\left[H_{\mu}, \sum_{\beta \in \Delta^{+}} f_{\beta}(y) e_{\beta}\right]=\left[H_{\mu}, y\right] .
$$

Hence $(E x)(y)=\Sigma_{\mu \in \Delta^{+}} c_{\mu} \theta\left(H_{\mu}\right)(y) \forall y \in n$. By (5.3.2)

$$
L_{\theta^{\mathfrak{n}}} x=0 \leftrightarrow E x=-c(0) x \leftrightarrow x=(1 /-c(0)) E x \quad \text { since } c(0) \neq 0
$$

by (5.3.1). Q.E.D.

Proposition 5.3.10 Choose a fixed ordering of $\Delta^{+}$so that $\Delta^{+}=\left\{a_{1}, \cdots, a_{l}\right.$, $\left.a_{l+1}, \cdots, a_{p}\right\}$ where $\pi=\left\{a_{1}, \cdots, a_{l}\right\}$. For $a_{j}$ nonsimple, $l+1 \leq j \leq p$, write (unicuely) $a_{j}=\sum_{i=1}^{l} n_{i}^{(j)} a_{i}, n_{i}^{(j)}=$ nonnegative integer. For $1 \leq i \leq l$ let

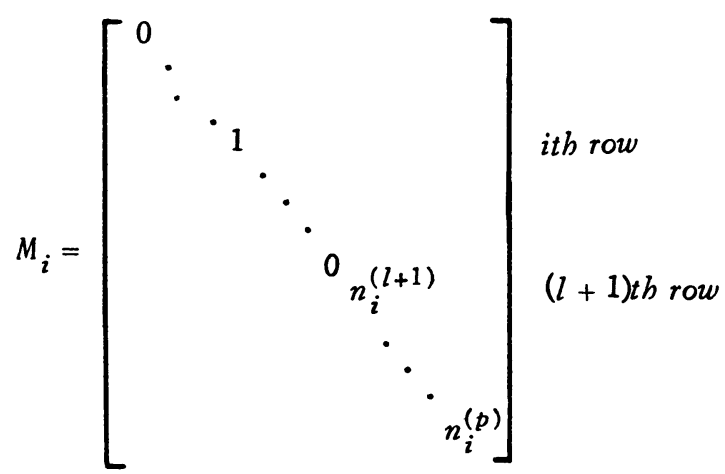

Then the matrices $\left\{M_{i}\right\}_{i=1}^{l}$ form a basis of $\left(\Lambda^{1} \bar{\pi} \otimes n\right)_{0}^{0}=\left\{x \in\left(\Lambda^{1} \bar{n} \otimes n\right)^{0} \mid L_{\theta^{n^{x}}} x=\right.$ 0\}. Therefore $\operatorname{dim}\left(\Lambda^{1} n \otimes n\right)_{0}^{0}=l=$ rank of $g$.

Proof. By Proposition 5.3.9, $x \in\left(\Lambda^{1} \bar{n} \otimes n\right)_{0}^{0}$ if and only if

$$
x=\sum_{a \in \Delta^{+}} c_{a} \theta\left(H_{a}\right), \quad c_{a} \in \mathbf{C} .
$$

Hence for each $\beta$ in $\Delta^{+}$

$$
x\left(e_{\beta}\right)=\sum_{a \in \Delta^{+}} c_{a}\left[H_{\alpha}, e_{\beta}\right]=\sum_{a \in \Lambda^{+}} c_{\alpha}(\beta, \alpha) e_{\beta}=\left(\beta, \sum_{\alpha \in \Delta^{+}} c_{a} \alpha\right) e_{\beta} .
$$

Therefore

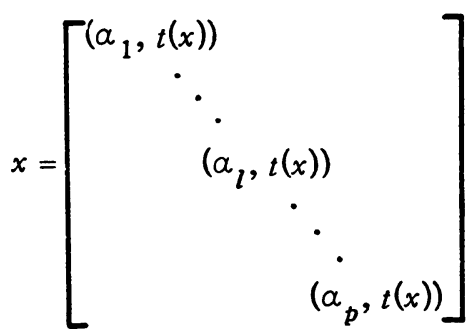


where we define $t(x)=\Sigma_{a \in \Delta^{+}} c_{a} \alpha \in \mathfrak{h}^{\prime}$. On the other hand

$$
\sum_{i=1}^{l}\left(\alpha_{i}, t(x)\right) M_{i}=\left[\begin{array}{c}
\left(\alpha_{1}, t(x)\right) \\
\cdot \\
\left(\alpha_{l}, t(x)\right) \\
\sum_{i=1}^{l} n_{i}^{(l+1)}\left(\alpha_{i}, t(x)\right) \\
\ddots \\
\\
\\
\\
\sum_{i=1}^{l} n_{i}^{(p)}\left(\beta_{i}, t(x)\right)
\end{array}\right]=x
$$

since $\left(\alpha_{j}, t(x)\right)=\sum_{i=1}^{l} n_{i}^{(j)}\left(\alpha_{i}, t(x)\right)$ for $l+1 \leq j \leq p$; see (5.3.11). Q.E.D.

At this point we are in position to state and prove the main result of $\S_{5}$ :

Theorem 5.3.12 (Kostant). Suppose $g$ is simple with rank greater than one. Let $\lambda$ be the unique highest root of the (irreducible) adjoint representation $\theta$ of $\mathrm{g}$ on 9 . Let $\bar{g}$ be a Cartan subalgebra of $g$ such that $\bar{G}=\overline{5}$ where conjugation on $g$ is defined by (2.2.2). Let $n=\Sigma_{a \in \Delta^{+}} g_{a}$ be the nilpotent subalgebra of 9 generated by the positive root spaces. Then the first derived cohomology space $H^{1}(n, n)$ has dimension (over $\mathrm{C}$ ) equal to twice the rank of $\mathrm{g}$ and as an $\mathrm{G}$ module

$$
H^{1}(n, n)=N^{0}(n)+\sum_{a \in \pi} N^{a}(n) \quad(\text { direct sum })
$$

where $\pi$ is a system of simple roots and:

(i) $N^{0}(n)$ is the subspace of derivations of $n$ of the form $\Sigma_{a \in \Delta^{+}}{ }^{c}{ }_{a} \theta\left(H_{a}\right)$, $c_{\alpha} \in \mathbf{C}$, with $H_{a}$ given by (4.2.3). The derivations $\theta\left(H_{\alpha}\right)$, where $\alpha$ varies over $\pi$, form a basis of $N^{0}(n)$; hence $\operatorname{dim}_{\mathrm{C}} N^{0}(n)=$ rank of $g$.

(ii) $N^{a}(n), \alpha \in \pi$, is a one dimensional subspace of derivations of $n$ with generator $\bar{e}_{\alpha} \otimes e_{S_{a} \lambda}$ where $S_{\alpha}$ is the simply Weyl reflection defined by a (see 4.2.11)), and $\left\{e_{\beta}\right\}_{\beta \in \Delta^{+}},\left\{\bar{e}_{\beta}\right\}_{\beta \in \Delta^{+}}$are orthonormal bases of $n$ and $\bar{n}=\Sigma_{\beta \in \Delta^{+}} g_{-}$; see (2.2.1). Thus $\bar{e}_{\alpha} \otimes e_{s_{\alpha} \lambda}: e_{\beta} \rightarrow \delta_{\alpha \beta} e_{s_{\alpha} \lambda}(\alpha, \beta) \in \pi \times \Delta^{+}$.

If the rank of $g$ is one then $H^{1}(n, n)=\mathrm{C} \theta\left(H_{\lambda}\right)$.

Proof. When $g$ has rank greater than one $\lambda \notin \pi$ so that $S_{\mu} \lambda \in \Delta^{+}$for every $\mu$ in $\pi$. By (5.1.8)

$$
H^{1}(n, n)=\left(\Lambda^{1} \bar{n} \otimes n\right)_{0}^{0}+\sum_{\text {distinct }}^{\gamma-\beta \neq 0 ; \gamma, \beta \in \Delta^{+}}\left(\Lambda^{1} \bar{n} \otimes n\right)_{0}^{\gamma-\beta} \text { (direct sum). }
$$

By Proposition 5.3.9, $\left(\Lambda^{1} \bar{n} \otimes n\right)_{0}^{0}=N^{0}(n)$. By Proposition 5.3.7, $\left(\Lambda^{1} \bar{\imath} \otimes n\right)_{0}^{\gamma-\beta}=$ $\{0\}$ for $\gamma, \beta \in \Delta^{+}, \gamma-\beta \neq 0$ unless $c(\gamma-\beta)=0$. By Lemma 5.2.4 and (5.3.1) the weights $\gamma-\beta, \gamma, \beta \in \Delta^{+}$, for which $c(\gamma-\beta)=0$ are precisely those of the form 
$S_{\mu} \lambda-\mu, \mu \in \pi$, and furthermore

$$
\left(\Lambda^{1} \overline{\mathfrak{n}} \otimes n\right)^{S_{\mu} \lambda-\mu}=\mathrm{C} \bar{e}_{\mu} \otimes e_{S_{\mu} \lambda} \quad \text { by (5.2.17). }
$$

If the rank of $g$ is one, $\pi=\{\lambda\}$ and $H^{1}(n, n)=N^{0}(n)=\mathbf{C} \theta\left(H_{\lambda}\right)$. Q.E.D.

6. Some key lemmas.

6.1 Before taking up the study of other cohomology groups we establish the following formulas which have slight independent interest. Again we assume that $g$ is simple, $\lambda$ is the highest root, and $2 \delta=\Sigma_{\beta \in \Delta^{+}} \beta$. We shall prove later
that $(\lambda, \lambda+2 \delta)=1$.

\section{Lemma 6.1.1}

(i)

$$
\sum_{\beta \in \Delta^{+}}\left[\bar{e}_{\beta},\left[e_{\beta}, e_{\alpha}\right]\right]=1 / 2[(\lambda, \lambda+2 \delta)-(2 \delta, \alpha)-(\alpha, \alpha)] e_{\alpha}
$$

for every $\alpha$ in $\Delta$.

$$
\sum_{\beta \in \Delta^{+} ; \beta-\alpha \epsilon \Delta^{-}}\left[e_{\beta}\left[\bar{e}_{\beta}, e_{\alpha}\right]\right]=(2 \delta-\alpha, \alpha) e_{a}
$$

for every $\alpha$ in $\Delta^{+}$(Griffiths and Schmid; cf. Lemma 3.1 of [2]).

(iii)

$$
\sum_{\beta \epsilon \Delta^{+} ; \beta-\alpha \epsilon \Delta^{+}}\left[\bar{e}_{\beta},\left[e_{\beta}, \bar{e}_{\alpha}\right]\right]=1 / 2[(\lambda, \lambda+2 \delta)-(2 \delta, \alpha)-(\alpha, \alpha)] \bar{e}_{a}
$$

for every $\alpha$ in $\Delta^{+}$.

Proof. By (4.3.3) and (4.3.5)

$$
\sum_{\beta \in \Delta^{+}} \theta\left(\bar{e}_{\beta}\right) \theta\left(e_{\beta}\right)+\sum_{i=1}^{l} \theta\left(\bar{H}_{i}\right) \theta\left(H_{i}\right)+\sum_{\beta \in \Delta^{+}} \theta\left(e_{\beta}\right) \theta\left(\bar{e}_{\beta}\right)=(\lambda, \lambda+2 \delta) 1
$$

or by (4.3.9)

$$
2 \sum_{\beta \in \Delta^{+}} \theta\left(\bar{e}_{\beta}\right) \theta\left(e_{\beta}\right)+\sum_{i=1}^{l} \theta\left(\bar{H}_{i}\right) \theta\left(H_{i}\right)+\sum_{\beta \in \Delta^{+}} \theta\left(H_{\beta}\right)=(\lambda, \lambda+2 \delta) 1 .
$$

For $\alpha$ in $\Delta$

$$
\sum_{i=1}^{l} \theta\left(\bar{H}_{i}\right) \theta\left(H_{i}\right) e_{a}=\sum_{i=1}^{l} \alpha\left(H_{i}\right) \alpha\left(\bar{H}_{i}\right) e_{\alpha}=(\alpha, \alpha) e_{\alpha .}
$$

by (4.3.10). Also

Therefore

$$
\sum_{\beta \in \Delta^{+}} \theta\left(H_{\beta}\right) e_{\alpha}=\sum_{\beta \in \Delta^{+}}(\alpha, \beta) e_{\alpha}=(\alpha, 2 \delta) e_{\alpha}
$$

$$
2 \sum_{\beta \in \Delta^{+}} \theta\left(\bar{e}_{\beta}\right) \theta\left(e_{\beta}\right) e_{\alpha}+(\alpha, \alpha) e_{\alpha}+(\alpha, 2 \delta) e_{a}=(\lambda, \lambda+2 \delta) e_{\alpha}
$$

for $\alpha$ in $\Delta$ which proves (i). To prove (ii) we introduce structure constants $N_{a, \beta}$ 
defined by

$$
\left[e_{\alpha}, e_{\beta}\right]=N_{a, \beta} e_{\alpha+\beta} \text { if } \alpha+\beta \epsilon \Delta
$$

and

$$
N_{a, \beta}=0 \text { if } \alpha+\beta \notin \Delta .
$$

By (4.2.10) and the invariance of the Killing form we have the standard relations

$$
N_{a+\beta,-a}=N_{-\beta, \alpha+\beta}=N_{-a,-\beta}
$$

if $\alpha, \beta, \alpha+\beta \in \Delta$. Of course

$$
N_{a, \beta}=-N_{\beta, a}
$$

Now given $a$ in $\Delta^{+}, \beta-a \in \Delta^{+}$if and only if $\beta=a+\gamma, \gamma \in \Delta^{+}$. Therefore since $2 a \notin \Delta^{+}$

$$
\begin{aligned}
\sum_{\beta \in \Delta^{+} ; \beta-a \in \Delta^{+}}\left[e_{\beta},\left[e_{\beta}, a\right]\right] & =\sum_{\gamma \in \Delta^{+} ; \gamma \neq a}\left[e_{a+\gamma},\left[\bar{e}_{a+\gamma}, e_{a}\right]\right] \\
& =\sum_{\gamma \in \Delta^{+} ; \gamma \neq a} N_{a+\gamma,-\gamma} N_{-a-\gamma, \alpha} e_{a} \\
& =\sum_{\gamma \in \Delta^{+} ; \gamma \neq a} N_{-\gamma,-a} N_{a, \gamma} e_{a}
\end{aligned}
$$

by (6.1.3); hence

$$
\sum_{\beta \in \Delta^{+} ; \beta-a \in \Delta^{+}}\left[e_{\beta},\left[\bar{e}_{\beta}, e_{\alpha}\right]\right]=\sum_{\beta \in \Delta^{+} ; \beta \neq \alpha} N_{\alpha, \beta} N_{-\beta,-\alpha} e_{\alpha}
$$

Next

$$
\sum_{\beta \in \Delta^{+} ; \beta-a \in \Delta^{-}}\left[e_{\beta},\left[\bar{e}_{\beta}, e_{\alpha}\right]\right]+\sum_{\beta \in \Delta^{+} ; \beta-\alpha \notin \Delta^{-}}\left[e_{\beta},\left[\bar{e}_{\beta}, e_{\alpha}\right]\right]=\sum_{\beta \in \Delta^{+}}\left[e_{\beta},\left[\bar{e}_{\beta}, e_{\alpha}\right]\right]
$$

or

$$
\begin{aligned}
\sum_{\beta \in \Delta^{+} ; \beta-a \in \Delta^{-}}\left[e_{\beta},\left[\bar{e}_{\beta}, e_{\alpha}\right]\right]+ & \sum_{\beta \in \Delta^{+} ; \beta-a \notin \Delta^{-} ; \beta \neq a}\left[e_{\beta},\left[\bar{e}_{\beta}, e_{\alpha}\right]\right]+\left[e_{\alpha},\left[\bar{e}_{\alpha}, e_{\alpha}\right]\right] \\
= & \sum_{\beta \in \Delta^{+} ; \beta \neq a}\left[e_{\beta},\left[\bar{e}_{\beta}, e_{\alpha}^{;}\right]\right]+\left[e_{\alpha},\left[\bar{e}_{\alpha}, e_{\alpha}\right]\right] .
\end{aligned}
$$

Consequently

$\sum_{\beta \in \Delta^{+} ; \beta-a \in \Delta^{-}}\left[e_{\beta},\left[\bar{e}_{\beta}, e_{\alpha}\right]\right] \doteq \sum_{\beta \in \Delta^{+} ; \beta \neq a}\left[e_{\beta},\left[\bar{e}_{\beta}, e_{\alpha}\right]\right]-\sum_{\beta \in \Delta^{+} ; \beta-a \in \Delta^{+}}\left[e_{\beta},\left[\bar{e}_{\beta}, e_{\alpha}\right]\right]$.

On the other hand $\left[e_{\beta},\left[\bar{e}_{\beta}, e_{\alpha}\right]\right]=N_{-\beta, a} N_{\beta,-\beta+a} e_{\alpha}$ so by (6.1.5) we have 


$$
\begin{aligned}
\sum_{\beta \in \Delta^{+} ; \beta-a \epsilon \Delta^{-}}\left[e_{\beta},\left[\bar{e}_{\beta}, e_{a}\right]\right] & =\sum_{\beta \in \Delta^{+} ; \beta \neq a}\left(N_{-\beta, a} N_{\beta,-\beta+a}-N_{a, \beta} N_{-\beta, \alpha}\right) e_{\alpha} \\
& =\sum_{\beta \in \Delta^{+} ; \beta \neq a}\left(N_{-\beta, a} N_{\beta, \alpha-\beta}-N_{\beta, a} N_{-\beta, \alpha+\beta}\right) e_{\alpha}
\end{aligned}
$$

by (6.1.3) and (6.1.4)

$$
\begin{aligned}
& =\sum_{\beta \in \Delta^{+} ; \beta \neq a}\left[e_{\beta},\left[\bar{e}_{\beta}, e_{\alpha}\right]\right]-\left[\bar{e}_{\beta},\left[e_{\beta}, e_{\alpha}\right]\right] \\
& =\sum_{\beta \in \Delta^{+} ; \beta \neq \alpha}\left(\left[e_{\beta},\left[\bar{e}_{\beta}, e_{\alpha}\right]\right]-\left[\bar{e}_{\beta},\left[e_{\beta}, e_{\alpha}\right]\right], \bar{e}_{\alpha}\right) e_{\alpha} \\
& =\sum_{\beta \in \Delta^{+} ; \beta \neq a}\left(\left[H_{\beta}, e_{\alpha}\right], \bar{e}_{\alpha}\right) e_{\alpha}=(2 \delta-\alpha, \alpha) e_{\alpha}
\end{aligned}
$$

by the Jacobi identity. This proves (ii). Let $\alpha \in \Delta^{+}$. We have

$$
\begin{aligned}
\sum_{\beta \in \Delta^{+}}\left[\bar{e}_{\beta},\left[e_{\beta}, \bar{e}_{\alpha}\right]\right]= & \sum_{\beta \in \Delta^{+} ; \beta-\alpha \in \Delta^{+}}\left[\bar{e}_{\beta},\left[e_{\beta}, \bar{e}_{\alpha}\right]\right] \\
& +\sum_{\beta \in \Delta^{+} ; \beta-\alpha \in \Delta^{+} ; \beta \neq \alpha}\left[\bar{e}_{\beta},\left[e_{\beta}, \bar{e}_{\alpha}\right]\right]+\left[\bar{e}_{\alpha},\left[e_{\alpha}, \bar{e}_{\alpha}\right]\right]
\end{aligned}
$$

or by (i), (ii), and (2.2.4)

$$
\begin{aligned}
1 / 2[(\lambda, \lambda+2 \delta)+(2 \delta, \alpha)-(\alpha, \alpha)] \bar{e}_{\alpha} \\
\quad=\sum_{\beta \in \Delta^{+} ; \beta-\alpha \in \Delta^{+}}\left[\bar{e}_{\beta},\left[e_{\beta}, \bar{e}_{\alpha}\right]\right]+(2 \delta-\alpha, \alpha) \bar{e}_{\alpha}+(\alpha, \alpha) \bar{e}_{\alpha} .
\end{aligned}
$$

This proves (iii).

Lemma 6.1.6 Let $\alpha \in \Delta^{+}$and let $\left(\alpha_{1}, \beta_{1}\right), \cdots,\left(\alpha_{m}, \beta_{m}\right)$ be the set of pairs of positive roots, ordered arbitrarily, such that $\alpha_{i}+\beta_{i}=\alpha$. Then

$$
\sum_{i=1}^{m}\left|\left(\bar{e}_{\alpha},\left[e_{\alpha_{i}}, e_{\beta_{i}}\right]\right)\right|^{2}=1 / 2[(2 \delta, \alpha)-(\alpha, \alpha)] \text {. }
$$

Proof. $\left[e_{a_{i}}, e_{\beta_{i}}\right]=\left(\bar{e}_{\alpha},\left[e_{a_{i}}, e_{\beta_{i}}\right]\right) e_{\alpha}$ and therefore

(see (2.2.4)). Hence

$$
\left.\left[\bar{e}_{\beta_{i}}, \bar{e}_{\alpha_{i}}\right]=\overline{\left[e_{\alpha_{i}}, e_{\beta_{i}}\right]}=\overline{\left(\bar{e}_{\alpha},\left[e_{\alpha_{i}}, e_{\beta_{i}}\right]\right.}\right) \bar{e}_{\alpha}
$$

$$
\left(\left[e_{\alpha_{i}}, e_{\beta_{i}}\right],\left[\bar{e}_{\beta_{i}}, \bar{e}_{\alpha_{i}}\right]\right)=\left|\left(\bar{e}_{\alpha},\left[e_{\alpha_{i}}, e_{\beta_{i}}\right]\right)\right|^{2}
$$

for each $i$. Now write

$$
\begin{aligned}
& {\left[e_{a_{i}}, e_{\beta_{i}}\right]=N_{\alpha_{i}, \beta_{i}} e_{\alpha}=N_{a-\beta_{i}, \beta_{i}} e_{a},}
\end{aligned}
$$

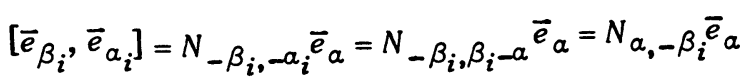


by (6.1.3). Therefore by (6.1.4) and (6.1.7)

$$
\sum_{i=1}^{m}\left|\left(\bar{e}_{\alpha},\left[e_{\alpha_{i}}, e_{\beta_{i}}\right]\right)\right|^{2}=\sum_{i=1}^{m} N_{\beta_{i}, a-\beta_{i}}{ }_{-\beta_{i}, \alpha^{\bullet}}
$$

Similarly

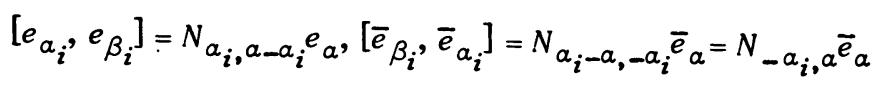

so that

$$
\sum_{i=1}^{m}\left|\left(\bar{e}_{a},\left[e_{a_{i}}, e_{\beta_{i}}\right]\right)\right|^{2}=\sum_{i=1}^{m} N_{-a_{i}, a} N_{a_{i}, a-a_{i}} .
$$

(6.1.8) and (6.1.9) imply that

$$
\begin{aligned}
2 \sum_{i=1}^{m}\left|\left(\bar{e}_{\alpha},\left[e_{\alpha_{i}}, e_{\beta_{i}}\right]\right)\right|^{2} & =\sum_{i=1}^{m} N_{\beta_{i}, a-\beta_{i}} N_{-\beta_{i}, a}+\sum_{i=1}^{m} N_{a_{i}, a-a_{i}} N_{-a_{i}, a} \\
& =\sum_{\gamma \in \Delta^{+} ; \gamma-a \in \Delta^{-}} N_{\gamma, a-\gamma^{N}} N_{-\gamma, a} \\
& =\sum_{\gamma \in \Delta^{+} ; \gamma-a \in \Delta^{-}}\left(\bar{e}_{\alpha},\left[e_{\gamma},\left[\bar{e}_{\gamma}, e_{\alpha}\right]\right]\right)=(2 \delta-\alpha, \alpha)
\end{aligned}
$$

by Lemma 6.1 .1 (ii). Q.E.D.

The next lemma is trivial.

Lemma 6.1.10 For every $H$ in $5, H=\Sigma_{\beta \in \Delta} \beta(H) H_{\beta}$; bence $H / 2=$ $\sum_{\beta \in \Delta^{+}} \beta(H) H_{\beta}$.

Proof. Let $H_{1}=\Sigma_{\beta \epsilon \Delta} \beta(H) H_{\beta}$. Now $H=H_{\xi}$ for some unique $\xi$ in $\xi^{\prime}$. For every $\gamma$ in $\Delta, \gamma\left(H_{1}\right)=\Sigma_{\beta \epsilon \Delta}(\beta, \xi)(\beta, \gamma)=(\xi, \gamma)=\gamma(H)$; see [5, p. 118]. Hence $H_{1}=H$.

7. Determination of the cohomology group $H^{1}(n, g / n)$.

7.1 Because $n$ is $\theta(\mathfrak{n})$ invariant there is a quotient representation $\theta^{\mathfrak{n}}$ of $n$ on $g / n$. Identify $g / n$ with $n^{\perp}=\mathfrak{G}+\bar{n}$. Then the action $\theta^{\mathfrak{n}}$ of $n$ on $\mathscr{G}+\bar{n}$ is given by

$$
\theta^{\mathfrak{n}}(x)=\left.P \theta(x)\right|_{\mathfrak{h}+\bar{\pi}}, \quad x \in \mathfrak{n},
$$

where $P=P^{\mathfrak{n} \perp}$ is the orthogonal projection of $g$ onto $n^{\perp}$; see Proposition 3.2.2.

We shall be interested in describing the derived space $H^{1}(n, \bar{G}+\bar{n})$ completely.

The Laplace operator $L_{\theta^{\mathfrak{n}}}$ for the representation $\theta^{\mathfrak{n}}$ is given by The orem 3.2.12:

$$
L_{\theta^{\mathfrak{n}}}=(1 \otimes P) L_{\left.\theta\right|_{\mathfrak{n}}}+E
$$


on $\Lambda \bar{n} \otimes(\bar{G}+\bar{n})$ where

$$
E=\sum_{\beta \in \Delta^{+}} \sum_{\gamma \in \Delta^{+}} \epsilon\left(\bar{e}_{\beta}\right) \iota\left(e_{\gamma}\right) \otimes P \theta\left(\bar{e}_{\gamma}\right)(1-P) \theta\left(e_{\beta}\right)
$$

$$
+1 \otimes \sum_{\beta \in \Delta^{+}} P \theta\left(\bar{e}_{\beta}\right)(P-1) \theta\left(e_{\beta}\right), \quad P=P^{n^{\perp}} \text {. }
$$

If $x \in \Lambda \bar{n} \otimes(\bar{G}+\bar{n})$ such that $L_{\theta^{n}} x=0$ then we shall say that $x$ is harmonic (or $\theta^{\mathfrak{n}}$-harmonic).

By (4.1.6) we have a representation $\beta^{(2)}$ of $\mathfrak{h}$ on $\Lambda \bar{n} \otimes(\mathfrak{G}+\bar{n})$ given by

$$
\beta^{(2)}(H)=\left.\theta \otimes \theta(H)\right|_{\Lambda \bar{n} \otimes(b+\bar{n})}, \quad H \in \mathfrak{G} .
$$

The elements $\bar{e}_{a} \otimes \bar{e}_{\beta}, \bar{e}_{\alpha} \otimes H_{j}$, where $\left\{H_{j}\right\}_{j=1}^{l}$ is an orthonormal basis of $\mathfrak{G}$ and $a, \beta \in \Delta^{+}$, form an orthonormal basis of $\Lambda^{1} \bar{n} \otimes(\bar{G}+\bar{n})$. Therefore the weights of $\left.\beta^{(2)}\right|_{\Delta \bar{n} \otimes(\mathfrak{b}+\bar{n})}$ are

$$
-\alpha, \quad \text { and }-\alpha-\beta, \quad \alpha, \beta \in \Delta^{+} \text {. }
$$

By Corollary 4.1 .7 the corresponding we ight spaces are $L_{\theta^{\pi}}$ invariant. Therefore

$$
H^{1}(\mathfrak{n}, \bar{G}+n)=\sum_{\text {distinct } \xi}\left(\Lambda^{1} \bar{n}, \otimes(\bar{G}+\bar{n})\right)_{0}^{\xi} \quad \text { (direct sum) }
$$

where $\xi$ is a weight $-\alpha$ or $-\alpha-\beta, a, \beta \in \Delta^{+}$, and $\left(\Lambda^{1} \bar{n} \otimes(\mathscr{G}+\bar{n})\right)_{0}^{\xi}$ is the space of harmonic vectors in $\left(\Lambda^{1} n \otimes(\bar{G}+\bar{n})\right)^{\xi}$.

7.2 The contribution to cohomology which the weights $-a-\beta, \alpha, \beta \in \Delta^{+}$, $\alpha+\beta \notin \Delta^{+}$, make is easier to ascertain. We shall therefore consider these weights first. If $-\alpha-\beta$ is such a weight, with $\alpha, \beta \in \Delta^{+}, \alpha+\beta$ not a root, then one sees immediately (because $\alpha+\beta$ is not a root) that the weight space $\left(\Lambda^{1} \bar{n} \otimes(\bar{G}+\bar{n})\right)^{-a-\beta}$ consists of vectors of the form

$$
x=\sum_{\mu+\nu=\alpha_{+} \beta} c_{\mu \nu} \bar{e}_{\mu} \otimes \bar{e}_{\nu}, \quad c_{\mu \nu} \in \mathbf{C}
$$

cf. $(5.1 .6)$.

By (2.4.1) the coboundary operator $d_{\theta^{n}}$ is the linear map from $L(n, \bar{G}+\bar{n})$ to $\Lambda^{2}(n, \bar{h}+\bar{n})$ given by

$$
\left(d_{\theta^{\mathfrak{n}}} x\right)\left(y_{1}, y_{2}\right)=\theta^{\mathfrak{n}}\left(y_{1}\right) x\left(y_{2}\right)-\theta^{\mathfrak{n}}\left(y_{2}\right) x\left(y_{1}\right)-x\left(\left[y_{1}, y_{2}\right]\right)
$$

where $y_{1}, y_{2} \in n, x \in L(n, \bar{h}+\bar{n})$ (the space of linear operators from $n$ to $\bar{G}+\bar{n}$ ). Hence, from (7.1.1), $d_{\theta^{n}} x=0$; i.e. $x$ is a cocycle, if and only if

$$
x\left(\left[y_{1}, y_{2}\right]\right)=P^{n^{\perp}}\left(\left[x\left(y_{1}\right), y_{2}\right]+\left[y_{1}, x\left(y_{2}\right)\right]\right)
$$

for every $y_{1}, y_{2}$ in $n$. 
Proposition 7.2.3 Let $\mu \in \Delta^{+}$. If $\bar{e}_{\mu} \otimes \bar{e}_{\mu}$ is a cocycle then $\mu \in \pi$.

Proof. Suppose $\mu=\mu_{1}+\mu_{2}$ where $\mu_{1}, \mu_{2} \in \Delta^{+}$and suppose $\bar{e}_{\mu} \otimes \bar{e}_{\mu}$ satisfies (7.2.2). Since $\mu_{1}+\mu_{2}$ is a root $\left[e_{\mu_{1}}, e_{\mu_{2}}\right]=a e_{\mu}$ where $a \neq 0, a \in \mathrm{C}$. Using (5.3.6) and (7.2.2) we get

$$
a \bar{e}_{\mu}=\operatorname{ax}\left(e_{\mu}\right)=P\left(\left[x\left(e_{\mu_{1}}\right), e_{\mu_{2}}\right]+\left[e_{\mu_{1}}, x\left(e_{\mu_{2}}\right)\right]\right)=P 0=0
$$

implies $a=0$ which is a contradiction. Q.E.D.

Lemma 7.2.4 Let $\mu, \nu \in \Delta^{+}$. Then

$$
\begin{gathered}
E \bar{e}_{\mu} \otimes \bar{e}_{\nu}=-1 / 2[(\lambda, \lambda+2 \delta)-(2 \delta, \nu)-(\nu, \nu)] \bar{e}_{\mu} \otimes \bar{e}_{\nu} \\
+\sum_{\substack{\beta \in \Delta^{+} ; \beta-\nu \epsilon \Delta^{+} \\
\beta-\left(\mu_{+} \nu\right) \epsilon\{0\} \cup \Delta^{-}}} \bar{e}_{\beta} \otimes\left[\bar{e}_{\mu},\left[e_{\beta}, \bar{e}_{\nu}\right]\right]
\end{gathered}
$$

where $E$ is the error term in (7.1.3).

Proof. By arguments similar to those in the proof of Lemma 5.2.1 one derives

$$
\begin{gathered}
E \vec{e}_{\mu} \otimes \cdot \bar{e}_{\nu}=-e_{\mu} \otimes \sum_{\beta \epsilon \Delta^{+} ; \beta-\nu \epsilon \Delta^{+}}\left[\bar{e}_{\beta},\left[e_{\beta}, \bar{e}_{\nu}\right]\right] \\
+\sum_{\substack{\beta \epsilon \Delta^{+} ; \beta-\nu \epsilon \Delta^{+} \\
\beta-(\mu+\nu) \epsilon\{0\} \cup \Delta^{-}}} \bar{e}_{\beta} \otimes\left[\bar{e}_{\mu},\left[e_{\beta}, \bar{e}_{\nu}\right]\right] .
\end{gathered}
$$

The proof is then concluded by the application of Lemma 6.1 .1 (iii).

.Corollary 7.2.5. If $\mu, \nu, \mu+\nu \in \Delta^{+}$then

$$
\begin{aligned}
& E \bar{e}_{\mu} \otimes \bar{e}_{\nu}=-1 / 2[(\lambda, \lambda+2 \delta)-(2 \delta, \nu)-(\nu, \nu)] \bar{e}_{\mu} \otimes \bar{e}_{\nu}+\bar{e}_{\mu+\nu} \otimes\left[\bar{e}_{\mu},\left[e_{\mu+\nu}, \bar{e}_{\nu}\right]\right] \\
& +\sum_{\substack{\beta \in \Delta^{+} ; \beta-\nu \epsilon \Delta^{+} \\
\beta-\left(\mu_{+} \nu\right) \epsilon \Delta^{-}}} \bar{e}_{\beta} \otimes\left[\bar{e}_{\mu},\left[e_{\beta}, \bar{e}_{\nu}\right]\right]
\end{aligned}
$$

Corollary 7.2.6 If $\mu \in \pi, \nu \in \Delta^{+}$and $\mu+\nu$ is not a root, then

$$
E \bar{e}_{\mu} \otimes \bar{e}_{\nu}=-1 / 2[(\lambda, \lambda+2 \delta)-(2 \delta, \nu)-(\nu, \nu)] \bar{e}_{\mu} \otimes \bar{e}_{\nu} \text {. }
$$

Proof. If $\beta \in \Delta^{+} \ni \beta-\nu \in \Delta^{+}$and $\beta-(\mu+\nu) \in\{0\} \cup \Delta^{-}$, then $\beta-$ $(\mu+\nu) \epsilon \Delta^{-}$since $\mu+\nu$ is not a root. Hence $\mu=(\beta-\nu)+[-(\beta-(\mu+\nu))] \not \pi$. Therefore

$$
\sum_{\substack{\beta \epsilon \Delta^{+} ; \beta-\nu \epsilon \Delta^{+} \\ \beta-(\mu+\nu) \epsilon\{0\} \cup \Delta^{-}}} \bar{e}_{\beta} \otimes\left[\bar{e}_{\mu},\left[e_{\beta}, \bar{e}_{\nu}\right]\right]=0 \text { if } \mu \in \pi \text { and } \mu+\nu \notin \Delta^{+} .
$$

Proposition 7.2.7 Let $\mu \in \Delta^{+}$. Then $\bar{e}_{\mu} \otimes \bar{e}_{\mu}$ is $\theta^{\mathfrak{n}}$-harmonic if and only if $\mu \in \pi$. 
Proof. If $\bar{e}_{\mu} \otimes \bar{e}_{\mu}$ is harmonic $\bar{e}_{\mu} \otimes \bar{e}_{\mu}$ is a cocycle in particular. Therefore $\mu \in \pi$ by Proposition 7.2.3. Conversely assume $\mu \in \pi$. Then because $(2 \delta, \mu)=(\mu, \mu)$ (see Proposition 4.2.13) and $2 \mu \notin \Delta$, Corollary 7.2.6 implies

$$
E \bar{e}_{\mu} \otimes \bar{e}_{\mu}=-1 / 2[(\lambda, \lambda+\delta)-2(\mu, \mu)] \bar{e}_{\mu} \otimes \bar{e}_{\mu} \text {. }
$$

By Theorem 4.3.8 and (7.1.2)

(7.2.9) $L_{\theta^{\mathfrak{n}}} x=0$ if and only if $E x=-c(\xi) x$ for $x \in\left(\Lambda \bar{n} \otimes n^{\perp}\right)^{\xi}$.

Of course $\bar{e}_{\mu} \otimes \bar{e}_{\mu} \in\left(\Lambda^{1} \bar{n} \otimes(\bar{g}+\bar{n})\right)^{-2 \mu}$. Also $c(-2 \mu)=1 / 2[(\lambda, \lambda+2 \delta)-2(\mu, \mu)]$, by (5.3.1) (again using $(2 \delta, \mu)=(\mu, \mu))$. Hence (7.2.8) and (7.2.9) show that $\bar{e}_{\mu} \otimes \bar{e}_{\mu}$ is harmonic.

We can now decide which weights of the form $-\alpha-\beta, \alpha, \beta \in \Delta^{+}, \alpha+\beta$ not a root, contribute to cohomology:

Proposition 7.2.9 Let $\alpha, \beta \in \Delta^{+}$such that $\alpha+\beta$ is not a root. Then $\left(\Lambda^{1} \bar{n} \otimes(\zeta+\bar{n})\right)_{0}^{-\alpha-\beta}=\{0\}$ unless $\alpha+\beta$ is of the form $\alpha+\beta=2 \alpha_{0}, \alpha_{0} \in \Delta^{+}$. On the other hand

$$
\left(\Lambda^{1} \bar{n} \otimes(\bar{G}+\bar{n})\right)_{0}^{-2 \alpha}= \begin{cases}\{0\} & \text { if } \alpha \notin \pi, \\ \mathrm{C} \bar{e}_{\alpha} \otimes \bar{e}_{\alpha} & \text { if } \alpha \in \pi .\end{cases}
$$

Proof. Let $x \in\left(\Lambda^{1} \bar{n} \otimes(\bar{G}+\bar{n})\right)^{-\alpha-\beta}$. By (7.2.1)

$$
x=\sum_{\mu+\nu=a+\beta} c_{\mu \nu} \bar{e}_{\mu} \otimes \bar{e}_{\nu}, \quad c_{\mu \nu} \in \mathbf{C} .
$$

Suppose that $x$ is harmonic. Then in particular $x$ satisfies (7.2.2). Given $\mu$, $\nu \in \Delta^{+}$such that $\mu+\nu=\alpha+\beta$ we take $y_{1}=e_{\mu}, y_{2}=e_{\nu}$ in (7.2.2). Then since $p^{\mathfrak{n}^{\perp}}=1$ on $\operatorname{k}$ we get

$$
x\left(\left[e_{\mu}, e_{\nu}\right]\right)=-c_{\mu \nu} H_{\nu}+c_{\nu \mu} H_{\mu}
$$

But $\left[e_{\mu}, e_{\nu}\right]=0$ since $\mu+\nu=\alpha+\beta$ is not a root. Therefore $c_{\mu \nu} H_{\nu}=c_{\nu \mu} H_{\mu}$ or $c_{\mu \nu} \nu=c_{\nu \mu} \mu$. If $\mu \neq \nu$ then we must have $c_{\mu \nu}=c_{\nu \mu}=0$ since $\mu, \nu$ are positive roots. Thus $x$ is zero if $\alpha+\beta$ is not of the form $2 \gamma$ for some $\gamma \in \Delta^{+}$. On the other hand if $\alpha+\beta=2 \gamma, \gamma \in \Delta^{+}$, then the argument just given shows that

$$
\left(\Lambda^{1} \bar{n} \otimes(\bar{G}+\bar{n})\right)_{0}^{-2 \gamma} \subset \mathrm{C} \bar{e}_{\gamma} \otimes \bar{e}_{\gamma} .
$$

However by Proposition 7.2.7 we have equality if and only if $\gamma \in \pi$. Q.E.D.

7.3 The problem of determining which weights of the form $-\alpha, \alpha \in \Delta^{+}$, contribute to cohomology will now be studied. We will show eventually that $\left(\Lambda^{1} \bar{n} \otimes n^{\perp}\right)^{-\alpha}=\{0\}, \alpha \in \Delta^{+}$, unless $\alpha$ has level one or two (i.e. $\alpha$ is simple or $\alpha$ is the sum of two simple roots). 
An arbitrary element $x$ in $\left(\Lambda^{1} \bar{n} \otimes n^{\perp}\right)^{-a}, a \in \Delta^{+}$, has the form

$$
x=\bar{e}_{a} \otimes H+\sum_{\mu, \nu \in \Delta^{+} ; \mu+\nu=a} c_{\mu \nu} \bar{e}_{\mu} \otimes \bar{e}_{\nu}
$$

where $H \in \mathfrak{G}$ and $c_{\mu \nu} \in \mathbf{C}$.

Lemma 7.3.2 For $\alpha \in \Delta^{+}, H \in \mathfrak{G}$

$$
E \bar{e}_{\alpha} \otimes H=-1 / 2 \bar{e}_{\alpha} \otimes H+\alpha(H) \bar{e}_{\alpha} \otimes H_{a}+\sum_{\beta \in \Delta^{+} ; \beta-\alpha \in \Delta^{-}} \beta(H) \bar{e}_{\beta} \otimes\left[e_{\beta}, \bar{e}_{\alpha}\right]
$$

where $E$ is the error term in (7.1.3).

Proof. By arguments similar to those given in the proof of Lemma 5.2.1 one derives

$$
E \bar{e}_{\alpha} \otimes H=-\bar{e}_{\alpha} \otimes \sum_{\beta \in \Delta^{+}} \beta(H) H_{\beta}+\alpha(H) \bar{e}_{\alpha} \otimes H_{a}+\sum_{\beta \in \Delta^{+} ; \beta-\alpha \in \Delta^{-}} \bar{e}_{\beta} \otimes \beta(H)\left[e_{\beta}, \bar{e}_{\alpha}\right] .
$$

Now apply Lemma 6.1.10. Q.E.D.

Corollary 7.3.3 For $\alpha \in \pi, H \in \mathfrak{G}$

$$
E \bar{e}_{\alpha} \otimes H=-1 / 2 \bar{e}_{\alpha} \otimes H+\alpha(H) \bar{e}_{\alpha} \otimes H_{\alpha} .
$$

Proposition 7.3.4 If $\bar{e}_{a} \otimes H$ is a cocycle where $\alpha \in \Delta^{+}, H \in \mathscr{G}$, then unless $H=0$, necessarily $\alpha \in \pi ; c f$. Proposition 7.2.3.

Proof. Suppose $\alpha=\beta+\gamma, \beta, \gamma \in \Delta^{+}$and $\bar{e}_{\alpha} \otimes H$ is a cocycle. Then $\left[e_{\beta}, e_{\gamma}\right]=a e_{a}$ where $\alpha \neq 0, a \in \mathrm{C}$, since $\beta+\gamma$ is a root. By (7.2.2), (5.3.6), $a H=\left(\bar{e}_{a} \otimes H\right)\left(\left[e_{\beta}, e_{\gamma}\right]\right)=P^{\mathfrak{n}^{\perp}} 0=0$. Hence $H=0$.

Up to this point we have not needed to observe that

$$
(\lambda, \lambda+2 \delta)=1 \text {. }
$$

This follows immediately if one knows that trace $C^{\theta}=\operatorname{dim} g\left(C^{\theta}=\right.$ Casimir operator); see (4.3.5). On the other hand one can compute trace $C^{\theta}$ directly from (4.3.3) using the root space decomposition of $g$, Lemma 6.1.1 (i), Lemma 6.1 .10 , and the formula

$$
C^{\theta}=2 \sum_{\beta \in \Delta^{+}} \theta\left(\bar{e}_{\beta}\right) \theta\left(e_{\beta}\right)+\sum_{i=1}^{l} \theta\left(\bar{H}_{i}\right) \theta\left(H_{i}\right)+\sum_{\beta \in \Delta^{+}} \theta\left(H_{\beta}\right) ;
$$

see (4.3.3). The result is that trace $C^{\theta}=2 p(\lambda, \lambda+2 \delta)+l$ where $p$ is the number of positive roots and $l=\operatorname{dim} \xi$. But since trace $C^{\theta}=(\lambda, \lambda+2 \delta) \operatorname{dim} g$ (by $(4.3 .5))=(\lambda, \lambda+2 \delta)(2 p+l)$ we get (7.3.5).

(5.3.1) and (7.3.5) imply

$$
2 c(\xi)=1-(2 \delta, \xi)-(\xi, \xi), \quad \xi \in \zeta^{\prime} .
$$

Proposition 7.3.7 Let $\alpha \in \Delta^{+}, H \in \mathfrak{G}-\{0\}$. Then $\bar{e}_{a} \otimes H$ is $\theta^{\mathfrak{n}}$-harmonic if and only if $\alpha \in \pi$ and $\alpha(H)=0$; cf. Proposition 7.2.7. 
Proof. Suppose $\bar{e}_{a} \otimes H$ is harmonic. Then $a \in \pi$ by Proposition 7.3.4 and

$$
E \bar{e}_{\alpha} \otimes H=-c(-a) \bar{e}_{\alpha} \otimes H
$$

by (7.2.9). By Corollary $7 \cdot 3.3$ and (7.3.6) this means that

$$
-1 / 2 \bar{e}_{a} \otimes H+\alpha(H) \bar{e}_{a} \otimes H_{a}=-1 / 2 \bar{e}_{a} \otimes H
$$

(again since $(2 \delta, \alpha)=(a, a)$ for $a \in \pi)$; i.e. $\alpha(H)=0$. Conversely if $\alpha \in \pi$ and $a(H)=0$ then $\bar{e}_{a} \otimes H$ satisfies (7.3.9) hence (7.3.8) and hence $\bar{e}_{a} \otimes H$ is harmonic by (7.2.9).

Corollary 7.3.10 Suppose $\alpha \epsilon \pi$. Then

$$
\begin{aligned}
\left(\Lambda^{1} n \otimes(\bar{G}+\bar{n})\right)_{0}^{-a} & =\left\{\bar{e}_{a} \otimes H \mid H \in \mathfrak{G}, a(H)=0\right\} \\
& =\bar{e}_{a} \otimes(\text { byperplane in } \bar{g} \text { defined by } a) .
\end{aligned}
$$

Therefore $\operatorname{dim}_{C}\left(\Lambda^{1} n \otimes(\bar{g}+\bar{n})\right)_{0}^{-a}=($ rank of $g)-1$.

Proof. By (7.3.1), $\left(\Lambda^{1} n \otimes(\bar{G}+\bar{n})\right)^{-a}=\left\{\bar{e}_{a} \otimes H \mid H \in \mathscr{G}\right\}$ when $\alpha \in \pi$. Therefore Corollary 7.3.10 follows from Proposition 7.3.7.

7.4 Corollary 7.3.10 describes of course the harmonic weight vectors for weights $-a$ where $a$ is a simple root. What is the situation for $\alpha$ nonsimple? We shall focus attention on this question now.

In (7.3.1) let

$$
z=\sum_{\mu, \nu \in \Delta^{+} ; \mu+\nu=\alpha} c_{\mu \nu} \bar{e}_{\mu} \otimes \bar{e}_{\nu}, \quad c_{\mu \nu} \in \mathbf{C},
$$

so that we have

$$
x=\bar{e}_{\alpha} \otimes H+z .
$$

If $P^{\bar{n}}, P^{b}$ are the orthogonal projections of $g$ onto $\bar{n}, \sqrt{g}$ respectively then

$$
P^{n \perp}=P^{\mathfrak{b}}+P^{n}
$$

by (4.2.9). Using (5.3.6), (7.4.2), (7.4.3), and (7.2.2), it follows easily that $x$ is a cocycle if and only if

$$
z\left(\left[y_{1}, y_{2}\right]\right)=P^{\bar{n}}\left(\left[z\left(y_{1}\right), y_{2}\right]+\left[y_{1}, z\left(y_{2}\right)\right]\right)
$$

and

$$
f_{a}\left(\left[y_{1}, y_{2}\right]\right) H=P^{y}\left(\left[z\left(y_{1}\right), y_{2}\right]+\left[y_{1}, z\left(y_{2}\right)\right]\right)
$$

for every $y_{1}, y_{2}$ in $n$.

Lemma 7.4.5 Suppose $\alpha \in \Delta^{+}$such that $\alpha=\alpha_{0}+\beta_{1}+\beta_{2}$ where $\alpha_{0}, \beta_{1}$, $\beta_{2}, \alpha_{0}+\beta_{1}, \beta_{1}+\beta_{2} \in \Delta^{+}$but $\alpha_{0}+\beta_{2}$ is not a root. Suppose that z given by (7.4.1) satisfies (7.4.4). Then $c_{a_{0} \beta_{1}+\beta_{2}}=-c_{\beta_{1}+\beta_{2} a_{0}}$

Proof. Let $\beta=\beta_{1}+\beta_{2} \in \Delta^{+}$. Then $\left[e_{\beta_{1}}, e_{\beta_{2}}\right]=\left(\bar{e}_{\beta},\left[e_{\beta_{1}}, e_{\beta_{2}}\right]\right) e_{\beta}$ implies 


$$
z\left(\left[e_{\beta_{1}}, e_{\beta_{2}}\right]\right)=\left(\bar{e}_{\beta},\left[e_{\beta_{1}}, e_{\beta_{2}}\right]\right) c_{\beta a_{0}} \bar{e}_{a_{0}} .
$$

Indeed

(7.4.6)

$$
z\left(e_{\beta}\right)=\sum_{\mu, \nu \in \Delta^{+} ; \mu+\nu=\alpha=\alpha_{0+\beta}} c_{\mu \nu} \delta_{\mu \beta^{e_{\nu}}}=c_{\beta \alpha_{0}} \bar{e}_{\alpha_{0}} .
$$

Similarly since $\alpha_{0}+\beta_{2}$ is not a root $z\left(e_{\beta_{1}}\right)=0$ while $a_{0}+\beta_{1} \in \Delta^{+}$implies

$$
z\left(e_{\beta_{2}}\right)=c_{\beta_{2} a_{0}+\beta_{1}} \bar{e}_{a_{0+}+\beta_{1}} \cdot
$$

By (7.4.4) we have then

$$
\begin{aligned}
\left(\bar{e}_{\beta},\left[e_{\beta_{1}}, e_{\beta_{2}}\right]\right) c_{\beta a_{0}} \bar{e}_{a_{0}} & =P^{\bar{n}}\left(\left[e_{\beta_{1}}, c_{\beta_{2} a_{0}+\beta_{1}} \bar{e}_{a_{0}+\beta_{1}}\right]\right) \\
& =c_{\beta_{2} a_{0}+\beta_{1}}\left[e_{\beta_{1}}, \bar{e}_{a_{0+\beta_{1}}}\right] .
\end{aligned}
$$

On the other hand $\left[e_{a_{0}}, e_{\beta_{2}}\right]=0$ since $\alpha_{0}+\beta_{2}$ is not a root. Therefore $z\left(\left[e_{a_{0}}, e_{\beta_{2}}\right]\right)=0$. Also $z\left(e_{a_{0}}\right)=c_{a_{0} \beta \bar{e}_{\beta}}$ so that by (7.4.4) and (7.4.7) again we derive

$$
0=P^{\bar{n}}\left(\left[c_{a_{0} \beta^{\bar{e}}}, e_{\beta_{2}}\right]+\left[e_{\alpha_{0}}, c_{\beta_{2} a_{0}+\beta_{1}} \bar{e}_{a_{0}+\beta_{1}}\right]\right)
$$

or

$$
c_{a_{0} \beta}\left[\bar{e}_{\beta}, e_{\beta_{2}}\right]=-c_{\beta_{2} a_{0+\beta_{1}}}\left[e_{a_{0}}, \bar{e}_{a_{0+\beta_{1}}}\right]
$$

or

$$
c_{a_{0} \beta^{\beta}}\left(e_{\beta_{1}},\left[\bar{e}_{\beta}, e_{\beta_{2}}\right]\right)=-c_{\beta_{2} a_{0}+\beta_{1}}\left(e_{\beta_{1}},\left[e_{a_{0}}, \bar{e}_{\alpha_{0}+\beta_{1}}\right]\right)
$$

or

$$
c_{a_{0} \beta}\left(\bar{e}_{\beta},\left[e_{\beta_{1}}, e_{\beta_{2}}\right]\right)=c_{\beta_{2} a_{0}+\beta_{1}}\left(e_{\alpha_{0}},\left[e_{\alpha_{0}+\beta_{1}}, e_{\beta_{1}}\right]\right)
$$

by the invariance of the Killing form. But by (7.4.8)

$$
c_{\beta a_{0}}\left(\bar{e}_{\beta},\left[e_{\beta_{1}}, e_{\beta_{2}}\right]\right)=c_{\beta_{2} \alpha_{0}+\beta_{1}}\left(e_{\alpha_{0}},\left[e_{\beta_{1}}, e_{a_{0}+\beta_{1}}\right]\right) \text {. }
$$

Since $\beta_{1}+\beta_{2}$ is a root $\left[e_{\beta_{1}}, e_{\beta_{2}}\right] \neq 0$ so $\left(\bar{e}_{\beta},\left[e_{\beta_{1}}, e_{\beta_{2}}\right]\right) \neq 0$. Therefore the lemma follows from (7.4.9) and (7.4.10).

Lemma 7.4.11 Suppose $a \in \Delta^{+}$such that $\alpha=\alpha_{0}+\beta_{0}$ where $\alpha_{0} \in \pi, \beta_{0} \in \Delta^{+}$. Suppose that $x$ given by $(7.4 .2)($ or $(7.3 .1))$ is harmonic. Then $\left(2 \delta, \beta_{0}\right) c_{a_{0} \beta_{0}}=$ $\left(a_{0}, a_{0}\right) c_{\beta_{0} a_{0}}$

Proof. By (7.2.9) $x$ is harmonic if and only if $E x=-c(-\alpha) x$. By $(5.3 .1)$ and

$$
c(\xi)=1 / 2[1-(2 \delta, \xi)-(\xi, \xi)]
$$

for every $\xi$ in $\xi^{\prime}$. By Corollary 7.2.5 and Lemma 7.3.2 
$(7.4 .13)$

$$
\begin{aligned}
& E x=-1 / 2 \bar{e}_{\alpha} \otimes H+\alpha(H) \bar{e}_{\alpha} \otimes H_{a}+\sum_{\beta \in \Delta^{+} ; \beta-a \epsilon \Delta^{-}} \beta(H) \bar{e}_{\beta} \otimes\left[e_{\beta}, \bar{e}_{a}\right] \\
& -\sum_{\mu, \nu \in \Delta^{+} ; \mu_{+} \nu=\alpha} c_{\mu \nu}{ }^{1 / 2}[1-(2 \delta, \nu)-(\nu, \nu)] \bar{e}_{\mu} \otimes \bar{e}_{\nu} \\
& +\sum_{\mu, \nu \in \Delta^{+} ; \mu_{+} \nu=a} c_{\mu \nu} \bar{e}_{\mu_{+} \nu} \otimes\left[\bar{e}_{\mu},\left[e_{\mu_{+} \nu}, \bar{e}_{\nu}\right]\right] \\
& +\sum_{\mu, \nu \in \Delta^{+} ; \mu+\nu=a} \sum_{\substack{\beta \in \Delta^{+} ; \beta-\nu \in \Delta^{+} \\
\beta-\alpha \in \Delta^{-}}} c_{\mu \nu} \bar{e}_{\beta} \otimes\left[\bar{e}_{\mu},\left[e_{\beta}, e_{\nu}\right]\right] .
\end{aligned}
$$

Keeping in mind that $\alpha_{0} \in \pi$ we get

(7.4.14) $(E x)\left(e_{a_{0}}\right)=\alpha_{0}(H)\left[e_{a_{0}}, \bar{e}_{\alpha}\right]-1 / 2 c_{a_{0} \beta_{0}}\left[1-\left(2 \delta, \beta_{0}\right)-\left(\beta_{0}, \beta_{0}\right)\right] \vec{e}_{\beta_{0}}$.

On the other hand

$$
(-c(-\alpha) x)\left(e_{a_{0}}\right)=-1 / 2[1+(2 \delta, \alpha)-(\alpha, \alpha)]_{a_{0} \beta_{0}} \bar{e}_{\beta_{0}} \cdot
$$

Since $\left[e_{a_{0}}, \bar{e}_{\alpha}\right]=\left(\left[e_{a_{0}}, \bar{e}_{a}\right], e_{\beta_{0}}\right) \bar{e}_{\beta_{0}},(7.4 .14)$ and (7.4.15) imply (for $x$ harmonic)

$$
\begin{array}{r}
\alpha_{0}(H)\left(\left[e_{a_{0}}, \bar{e}_{\alpha}\right], e_{\beta_{0}}\right)-1 / 2 c_{a_{0} \beta_{0}}\left[1-\left(2 \delta, \beta_{0}\right)-\left(\beta_{0}, \beta_{0}\right)\right] \\
=-1 / 2 c_{a_{0} \beta_{0}}[1+(2 \delta, \alpha)-(\alpha, \alpha)] .
\end{array}
$$

Now take $y_{1}=e_{a_{0}}, y_{2}=e_{\beta_{0}}$ in (7.4.4). The result is that

$$
\left(\bar{e}_{\alpha},\left[e_{a_{0}}, e_{\beta_{0}}\right]\right) H=-c_{a_{0} \beta_{0}} H_{\beta_{0}}+c_{\beta_{0} \alpha_{0}} H_{a_{0}} \cdot
$$

Hence

(7.4.18) $\left(\bar{e}_{\alpha},\left[e_{a_{0}}, e_{\beta_{n}}\right]\right) \alpha_{0}(H)=-c_{\alpha_{0} \beta_{0}}\left(\alpha_{0}, \beta_{0}\right)+c_{\beta_{0} a_{0}}\left(\alpha_{0}, \alpha_{0}\right)$.

Since $\left(\left[e_{a_{0}}, \bar{e}_{\alpha}\right], e_{\beta_{0}}\right)=-\left(\bar{e}_{a},\left[e_{\alpha_{0}}, e_{\beta_{0}}\right]\right),(7.4 .16)$ and (7.4.18) imply that

$$
\begin{array}{r}
c_{a_{0} \beta_{0}}\left(\alpha_{0}, \beta_{0}\right)-c_{\beta_{0} \alpha_{0}}\left(\alpha_{0}, \alpha_{0}\right)-1 / 2 c_{a_{0} \beta_{0}}\left[1-\left(2 \delta, \beta_{0}\right)-\left(\beta_{0}, \beta_{0}\right)\right] \\
=-1 / 2 c_{a_{0} \beta_{0}}[1+(2 \delta, \alpha)-(\alpha, \alpha)] .
\end{array}
$$

Using the fact that $\alpha=\alpha_{0}+\beta_{0}$ and $\left(2 \delta, \alpha_{0}\right)=\left(\alpha_{0}, \alpha_{0}\right)$ (for $\left.\alpha_{0} \in \pi\right)$ we get the desired results.

We can now prove the following important

Proposition 7.4.19 Suppose $\alpha \in \Delta^{+}$such that $\alpha=\alpha_{0}+\beta_{1}+\beta_{2}$ where $\alpha_{0} \in \pi$, $\beta_{1}, \beta_{2}, a_{0}+\beta_{1}, \beta_{1}+\beta_{2} \in \Delta^{+}$but $\alpha_{0}+\beta_{2}$ is not a root. Then $\left(\Lambda^{1} \bar{n} \otimes(\bar{g}+\bar{n})\right)_{0}^{-\alpha}=\{0\}$.

Proof. Let $x=\bar{e}_{a} \otimes H+z \in\left(\Lambda^{1} \bar{n} \otimes(\bar{G}+\bar{n})\right)_{0}^{-a}$ where $z$ is given by (7.4.1). Since $x$ is a cocycle in particular $z$ :satisfies (7.4.4). Let $\beta=\beta_{1}+\beta_{2} \in \Delta^{+}$. 
Then by, Lemma 7.4.5, $c_{a_{0} \beta}=-c_{\beta a_{0}}$ and, by Lemma 7.4.11, $(2 \delta, \beta) c_{a_{0} \beta}=$ $\left(a_{0}, \alpha_{0}\right) c_{\beta a_{0}}$. That is $(2 \delta, \beta) c_{a_{0} \beta}=-\left(a_{0}, a_{0}\right) c_{a_{0} \beta}$ implies $c_{a_{0} \beta}=0$; otherwise $(2 \delta, \beta)=-\left(\alpha_{0}, \alpha_{0}\right)<0$ is a contradiction; one knows that $(2 \delta, \beta)>0$ for every $\beta$ in $\Delta^{+}$. By (7.4.17) then $H=0$ so that $x=z$. On the other hand noting that the derivation of (7.4.17) was independent of the simplicity of $\alpha_{0}$ we have (by (7.4.17))

$$
0=-c_{\mu \nu} H_{\nu}+c_{\nu \mu} H_{\mu}
$$

for $\mu, \nu \in \Delta^{+}$such that $\mu+\nu=\alpha$ or $c_{\mu \nu} \nu=c_{\nu \mu} \mu$. But $\nu \neq \mu$; otherwise $2 \mu=$ $a \in \Delta$. Therefore $c_{\mu \nu}=c_{\nu \mu}=0$ for $\mu+\nu=\alpha$; or $z=0$. Q.E.D.

A simple Lie algebra $g$ will be said to be simply laced if all its roots have the same length. Such Lie algebras are precisely the following: $A_{l}(l \geq 1)$, $D_{l}(l \geq 4), E_{6}, E_{7}$, and $E_{8^{\circ}}$. The nonsimply laced $g$ are therefore $B_{l}(l \geq 2)$, $C_{l}(l \geq 3), G_{2}$, and $F_{4}$.

Scholium 7.4.20 Suppose $g$ is nonsimply laced. Let $\alpha \in \Delta^{+}$be of level $\geq 3$ (i.e. $\alpha \notin \pi$ and $\alpha$ is not the sum of two simple roots). Then $\alpha=\alpha_{0}+\beta_{1}+\beta_{2}$ where $\alpha_{0} \in \pi, \beta_{1}, \beta_{2}, \alpha_{0}+\beta_{1}, \beta_{1}+\beta_{2} \in \Delta^{+}$but $\alpha_{0}+\beta_{2}$ is not a root.

Is the scholium true for all simply laced $g$ as well? Maybe it is. We shall not need it for such $g$ so we have not bothered to check all cases.

The proof is empirical. As an illustration we shall consider the case $g=$ $B_{l}$. Let $\pi=\left\{a_{1}, \cdots, a_{l}\right\}$. Then $\Delta^{+}=\left\{\omega_{i}, \omega_{i} \pm \omega_{j}\right\}$ where $1 \leq i<j \leq l$ and

$$
\begin{aligned}
\omega_{i} & =a_{i+1}+a_{i+2}+\cdots+a_{l}+a_{1}, \\
\omega_{i}-\omega_{j} & =a_{i+1}+\cdots+a_{j}, \\
\omega_{i}+\omega_{j} & =a_{i+1}+\cdots+a_{j}+2 a_{j+1}+\cdots+2 \alpha_{l}+2 a_{1} .
\end{aligned}
$$

Moreover

$$
\begin{gathered}
\text { level } \omega_{i}=l-i+1 \\
\text { level } \omega_{i}-\omega_{j}=j-i \\
\text { level } \omega_{i}+\omega_{j}=2 l-i-j+2
\end{gathered}
$$

We can write $\omega_{i}=a_{i+1}+\left(a_{i+2}+\cdots+a_{l}\right)+a_{1}$ where $\alpha_{i+2}+\cdots+a_{l}=\omega_{i+1}-$ $\omega_{l} \in \Delta^{+}$for $i+1<l$ which is the case when the level of $\omega_{i} \geq 3$. Also $a_{i+1}+$ $\left(a_{i+2}+\cdots+a_{l}\right),\left(a_{i+2}+\cdots+a_{l}\right)+a_{1} \in \Delta^{+}$but $a_{i+1}+a_{1}$ is not a root. Next consider the roots $\omega_{i}-\omega_{j}$. The level of $\omega_{i}-\omega_{j}(=j-i)$ is greater than or equal to 3 if and only if $i+3 \leq j$. First assume $i+3<j$. Then $a_{i+2}+\cdots+a_{j-1} \in \Delta^{+}$ and we can write $\omega_{i}-\omega_{j}=\alpha_{i+1}+\left(a_{i+2}+\cdots+\alpha_{j-1}\right)+\alpha_{j}$ where $\alpha_{i+1}+$ 
$\left(a_{i+2}+\cdots+a_{j-1}\right),\left(a_{i+2}+\cdots+a_{j-1}\right)+a_{j} \epsilon \Delta^{+}$but $a_{i+1}+a_{j}$ is not a root. On the other hand if $i+3=j$ then $\omega_{i}-\omega_{j}=a_{i+1}+a_{i+2}+\alpha_{i+3}$ where $\alpha_{i+1}+a_{i+2}$, $a_{i+2}+a_{i+3} \in \Delta^{+}$but $a_{i+1}+a_{i+3}$ is not a root. Finally we consider the roots $\omega_{i}+\omega_{j}$. These have level $\geq 3$ if and only if $i \leq 2 l-1-j$. First assume $j<l$. Then $a_{j+1}+2 a_{j+2}+\cdots+2 a_{l}+2 \alpha_{1} \in \Delta^{+}$and we can write

$$
\omega_{i}+\omega_{j}=a_{j+1}+\left(a_{i+1}+\cdots+a_{j}\right)+\left(a_{j+1}+2 a_{j+2}+\cdots+2 \alpha_{l}+2 \alpha_{1}\right)
$$

where $a_{j+1}+\left(a_{i+1}+\cdots+a_{j}\right),\left(a_{i+1}+\cdots+a_{j}\right)+\left(a_{j+1}+2 a_{j+2}+\cdots+2 a_{l}+2 a_{1}\right) \epsilon$ $\Delta^{+}$but $a_{j+1}+\left(a_{j+1}+2 a_{j+2}+\cdots+2 \alpha_{l}+2 \alpha_{1}\right)$ is not a root. If $j=l$, then $i \leq 2 l-1-j$ implies $i<l$. Write

$$
\omega_{i}+\omega_{j}=a_{1}+\left(a_{i+1}+\cdots+a_{l}\right)+a_{1}
$$

where $a_{1}+\left(a_{i+1}+\cdots+a_{l}\right) \in \Delta^{+}$but $2 \alpha_{1}$ is not a root. The case $g=C_{l}$ is handled in an entirely similar manner. We leave this case and the case $g=F_{4}$ to the reader. Of course the case $g=G_{2}$ is trivial.

By virtue of Proposition 7.4.19 we have

Corollary 7.4.21 Suppose $g$ is of type $B_{l}(l \geq 2), C_{l}(l \geq 3), G_{2}$ or $F_{4}$. Then if $a \in \Delta^{+}$has level $\geq 3,\left(\Lambda^{1} \overline{\mathrm{n}} \otimes(\bar{G}+\overline{\mathrm{n}})\right)_{0}^{-\alpha}=\{0\}$.

We shall prove that Corollary 7.4.21 is valid for all simply laced $g$ also. This would follow if the above scholium were extended to such g. However we shall proceed in an independent direction.

By (2.5.6), (3.1.10), and the fact that $c^{*}=\partial$ we have

$$
\left(d_{\left.\theta\right|_{\mathfrak{n}}}\right)^{*}=\partial \otimes 1+\sum_{\beta \in \Delta^{+}} c\left(e_{\beta}\right) \otimes \theta\left(\bar{e}_{\beta}\right)
$$

on $\Lambda \bar{n} \otimes g$. Moreover $\partial \Lambda^{1} g=0$ so that

$$
\left(d_{\left.\theta\right|_{\mathfrak{n}}}\right)^{*} \bar{e}_{a} \otimes H=1 \otimes\left[\bar{e}_{a}, H\right], \quad\left(d_{\left.\theta\right|_{\mathfrak{n}}}\right)^{*} \bar{e}_{\mu} \otimes \bar{e}_{\nu}=1 \otimes\left[\bar{e}_{\mu}, \bar{e}_{\nu}\right]
$$

for $a, \mu, \nu \in \Delta^{+}, H \in \mathfrak{K}$. Therefore (3.2.7) and (7.3.1) imply that

$$
\left(d_{\theta^{n}}\right)^{*} x=0
$$

if and only if

$$
\alpha(H) \bar{e}_{a}+\sum_{\mu, \nu \in \Delta^{+} ; \mu+\nu=a} c_{\mu \nu}\left[\bar{e}_{\mu}, \bar{e}_{\nu}\right]=0 .
$$

Proposition 7.4.25 Suppose $g$ is simply laced (i.e. $g$ is of type $A_{l}(l \geq 1)$, $D_{l}(l \geq 4), E_{6}, E_{7}$ or $\left.E_{8}\right)$ and $a \in \Delta^{+}$has level $\geq 3$. Then $\left(\Lambda^{1} \bar{n} \otimes(\bar{G}+\bar{n})\right)_{0}^{\alpha}=\{0\}$.

Proof. Let $\mu, \nu \in \Delta^{+}$such that $\mu+\nu=a$. Then because all roots have the same length we have $(\alpha, \alpha)=(\mu, \mu)+2(\mu, \nu)+(\nu, \nu)=2(\mu, \nu)+2(\alpha, \alpha)$ or 
(7.4.26)

$$
(\mu, \nu)=(a, a) /-2 \text {. }
$$

Suppose that $x$ given by (7.3.1) is harmonic. Recall that the derivation of (7.4.17) did not depend on the simplicity of $\alpha_{0}$. Therefore by (7.4.17), (7.4.26), and the fact that all roots have the same length

(7.4.27) $\quad \alpha(H)\left(\bar{e}_{\alpha},\left[e_{\mu}, e_{\nu}\right]\right)=-c_{\mu \nu}(\alpha, \nu)+c_{\nu \mu}(\alpha, \mu)=\left(c_{\nu \mu}-c_{\mu \nu}\right)(\alpha, \alpha) / 2$ for $\mu, \nu \in \Delta^{+}$such that $\mu+\nu=a$. Let $\left\{\left(\mu_{i}, \nu_{i}\right)\right\}_{i=1}^{m}$ be the set of pairs of positive roots, arbitrarily ordered, for which $\mu_{i}+\nu_{i}=\alpha$. Then by (7.4.24)

$$
0=a(H) \bar{e}_{a}+\sum_{i=1}^{m}\left(c_{\nu_{i} \mu_{i}}-c_{\mu_{i} \nu_{i}}\right)\left[\bar{e}_{\nu_{i}}, \bar{e}_{\mu_{i}}\right]
$$

or

$$
\begin{aligned}
0 & =\alpha(H)+\sum_{i=1}^{m}\left(c_{\nu_{i} \mu_{i}}-c_{\mu_{i} \nu_{i}}\right)\left(e_{\alpha},\left[\bar{e}_{\nu_{i}}, \bar{e}_{\mu_{i}}\right]\right) \\
& =\alpha(H)+\frac{2 \alpha(H)}{(\alpha, \alpha)} \sum_{i=1}^{m}\left|\left(\bar{e}_{\alpha},\left[\bar{e}_{\nu_{i}}, \bar{e}_{\mu_{i}}\right]\right)\right|^{2}
\end{aligned}
$$

by (7.4.27). Hence $a(H)=0$ and by (7.4.27) we have

$$
c_{\mu_{i} \nu_{i}}=c_{\nu_{i} \mu_{i}}, \quad 1 \leq i \leq m .
$$

We claim that $c_{\mu_{i} \nu_{i}}=0$ if $\mu_{i} \in \pi$ and $\nu_{i} \in \Delta^{+}-\pi$. Indeed, by Lemma 7.4.11 and (7.4.28), $\left(2 \delta, \nu_{i}\right) c_{\mu_{i} \nu_{i}}=\left(\mu_{i}, \mu_{i}\right) c_{\nu_{i} \mu_{i}}=\left(\nu_{i}, \nu_{i}\right) c_{\mu_{i} \nu_{i}}$ so $\left(2 \delta, \nu_{i}\right)=\left(\nu_{i}, \nu_{i}\right)$ if $c_{\mu_{i} \nu_{i}} \neq 0$. But then $\nu_{i} \in \pi$ by Proposition 4.2.13. Now if $a$ has level $\geq 3$, then we write

$$
\begin{aligned}
& x=\bar{e}_{a} \otimes H+\sum_{i=1}^{m} c_{\mu_{i} \nu} \bar{e}_{i} \otimes \bar{e}_{\nu_{i}}+\sum_{i=1}^{m} c_{\nu_{i} \mu_{i}} \bar{e}_{\nu_{i}} \otimes \bar{e}_{\mu_{i}}
\end{aligned}
$$

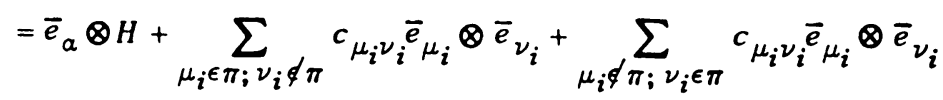

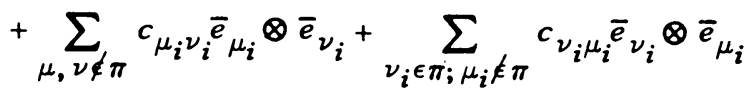

$$
\begin{aligned}
& +\sum_{\nu_{i} \notin \pi ; \mu_{i} \in \pi} c \nu_{\nu_{i} \mu_{i}} \bar{e}_{\nu_{i}} \otimes \bar{e}_{\mu_{i}}+\sum_{\nu_{i} \notin \pi_{i} \mu_{i} \notin \pi} c_{\nu_{i} \mu_{i}} \bar{e}_{\nu_{i}} \otimes \bar{e}_{\mu_{i}}
\end{aligned}
$$

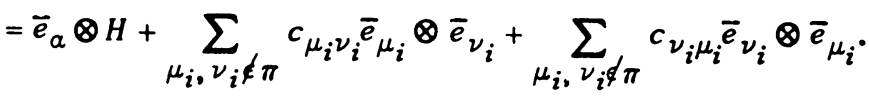

Hence $x\left(e_{\gamma}\right)=0$ for every $y \in \pi$. Therefore $x=0$ by (7.2.2). Q.E.D.

Corollary 7.4.21 and Proposition 7.4.25 imply

Corollary 7.4.29 $\left(\Lambda^{1} \bar{n} \otimes(\mathscr{G}+\mathfrak{n})\right)_{0}^{-a}=\{0\}$ if $a \in \Delta^{+}$has level $\geq 3$. 
7.5 The harmonic weight vectors defined by positive roots of level two are described by

Proposition 7.5.1 Suppose $a \in \Delta^{+}$such that $\alpha=\alpha_{1}+\alpha_{2}, \alpha_{1}, \alpha_{2} \in \pi$. Then $\left(\Lambda^{1} \bar{n} \otimes(\bar{G}+\bar{n})\right)_{0}^{-a}$ is one dimensional and is spanned by the barmonic vector

$$
\frac{\bar{e}_{a}}{\left(\bar{e}_{\alpha}\left[e_{a_{1}}, e_{a_{2}}\right]\right)} \otimes\left(\frac{\left(a_{2}, a_{2}\right)}{\left(\alpha_{1}, a_{2}\right)} H_{a_{1}}-H_{a_{2}}\right)+\bar{e}_{a_{1}} \otimes \bar{e}_{a_{2}}+\frac{\left(a_{2}, \alpha_{2}\right)}{\left(\alpha_{1}, \alpha_{1}\right)} \bar{e}_{a_{2}} \otimes \bar{e}_{a_{1}} \text {. }
$$

Proof. Suppose $x \in\left(\Lambda^{1} \bar{n} \otimes(\bar{\zeta}+\bar{n})\right)_{0}^{-\alpha}$. By (7.3.1)

$$
x=\bar{e}_{a} \otimes H+a \bar{e}_{a_{1}} \otimes \bar{e}_{a_{2}}+b \bar{e}_{a_{2}} \otimes \bar{e}_{a_{1}}
$$

where $H \in \zeta, a, b \in \mathbf{C}$. By Lemma 7.4.11 we must have $\left(2 \delta, \alpha_{2}\right) a=\left(a_{1}, \alpha_{1}\right) b$ or

$$
b=\left(\left(\alpha_{2}, \alpha_{2}\right) /\left(\alpha_{1}, \alpha_{1}\right)\right) a
$$

since $\alpha_{1}, \alpha_{2} \in \pi \cdot(7.4 .17)$ and (7.5.2) imply

$$
H=\frac{a}{\left(\bar{e}_{\alpha},\left[e_{a_{1}}, e_{\alpha_{2}}\right]\right)}\left(\frac{\left(\alpha_{2}, \alpha_{2}\right)}{\left(\alpha_{1}, \alpha_{1}\right)} H_{a_{1}}-H_{a_{2}}\right) \text {. }
$$

This shows that $\left(\Lambda^{1} \bar{n} \otimes(\bar{G}+\bar{n})\right)_{0}^{-a} \subset C x_{a}$ where

$$
x_{\alpha}=\frac{\bar{e}_{a}}{\left(\bar{e}_{\alpha},\left[e_{a_{1}}, e_{a_{2}}\right]\right)} \otimes\left(\frac{\left(a_{2}, a_{2}\right)}{\left(\alpha_{1}, a_{1}\right)} H_{a_{1}}-H_{a_{2}}\right)+\bar{e}_{a_{1}} \otimes \bar{e}_{a_{2}}+\frac{\left(a_{2}, a_{2}\right)}{\left(a_{1}, a_{1}\right)} \bar{e}_{a_{2}} \otimes \bar{e}_{a_{1}} .
$$

The proof is therefore reduced to showing that $x_{a}$ is indeed harmonic. By Lemma 7.3.2, Corollary 7.2.5, and (7.3.5)

$$
\begin{aligned}
& E x_{a}=\frac{-\bar{e}_{a}}{2\left(\bar{e}_{\alpha},\left[e_{\alpha_{1}}, e_{\alpha_{2}}\right]\right)} \otimes\left(\frac{\left(\alpha_{2}, \alpha_{2}\right)}{\left(\alpha_{1}, \alpha_{1}\right)} H_{\alpha_{1}}-H_{\alpha_{2}}\right) \\
& +\left(\frac{\left(\alpha_{2}, \alpha_{2}\right)}{\left(\alpha_{1}, \alpha_{1}\right)}\left(\alpha, \alpha_{1}\right)-\left(\alpha, \alpha_{2}\right)\right) \frac{\bar{e}_{a}}{\left(\bar{e}_{\alpha},\left[e_{a_{1}}, e_{a_{2}}\right]\right)} \otimes H_{\alpha} \\
& +\left(\frac{\left(\alpha_{2}, \alpha_{2}\right)}{\left(\alpha_{1}, \alpha_{1}\right)}\left(\alpha_{1}, \alpha_{1}\right)-\left(\alpha_{1}, \alpha_{2}\right)\right) \frac{\bar{e}_{a_{1}} \otimes\left[e_{\alpha_{1}}, \bar{e}_{a}\right]}{\left(\vec{e}_{\alpha},\left[e_{\alpha_{1}}, e_{\alpha_{2}}\right]\right)} \\
& +\left(\frac{\left(\alpha_{2}, a_{2}\right)}{\left(\alpha_{1}, \alpha_{1}\right)}\left(a_{2}, a_{1}\right)-\left(\alpha_{2}, a_{2}\right)\right) \frac{\bar{e}_{a_{2}} \otimes\left[e_{\alpha_{2}}, \bar{e}_{\alpha}\right]}{\left(\bar{e}_{\alpha},\left[e_{\alpha_{1}}, e_{a_{2}}\right]\right)} \\
& -1 / 2\left[1-2\left(a_{2}, a_{2}\right)\right] \bar{e}_{a_{1}} \otimes \bar{e}_{a_{2}}+\bar{e}_{\alpha} \otimes\left[\bar{e}_{a_{1}},\left[e_{\alpha}, \bar{e}_{a_{2}}\right]\right] \\
& +\frac{\left(a_{2}, \alpha_{2}\right)}{\left(\alpha_{1}, \alpha_{1}\right)}\left(-1 / 2\left[1-2\left(\alpha_{1}, \alpha_{1}\right)\right] \bar{e}_{\alpha_{2}} \otimes \bar{e}_{a_{1}}+\bar{e}_{\alpha} \otimes\left[\bar{e}_{a_{2}},\left[e_{a}, \bar{e}_{a_{1}}\right]\right]\right) \text {. }
\end{aligned}
$$


Now $\left[e_{a_{1}}, \bar{e}_{a}\right]=\left(e_{a_{2}},\left[e_{a_{1}}, \bar{e}_{a}\right]\right) \bar{e}_{a_{2}}=\left(\bar{e}_{a},\left[e_{a_{2}}, e_{a_{1}}\right]\right) \bar{e}_{a_{2}}$. Similarly $\left[e_{a_{2}}, \bar{e}_{a}\right]=\left(\bar{e}_{a},\left[e_{a_{1}}, e_{a_{2}}\right]\right) \bar{e}_{a_{1}}$. Of course $H_{a}=H_{a_{1}}+H_{a_{2}}$. Therefore

$$
\begin{aligned}
E x_{a}= & {\left[-1 / 2+\left(a_{1}, a_{2}\right)\right]\left(\bar{e}_{a_{1}} \otimes \bar{e}_{a_{2}}+\frac{\left(a_{2}, a_{2}\right)}{\left(a_{1}, a_{1}\right)} \bar{e}_{a_{2}} \otimes \bar{e}_{a_{1}}\right) } \\
& -\frac{1}{2} \frac{\bar{e}_{a}}{\left(\bar{e}_{a},\left[e_{a_{1}}, e_{\alpha_{2}}\right]\right)} \otimes\left(\frac{\left(a_{2}, a_{2}\right)}{\left(a_{1}, a_{1}\right)} H_{a_{1}}-H_{a_{2}}\right) \\
& +\left[\frac{\left(a_{2}, \alpha_{2}\right)}{\left(a_{1}, \alpha_{1}\right)}\left(a_{1}, \alpha_{2}\right)-\left(a_{1}, a_{2}\right)\right] \frac{\bar{e}_{a}}{\left(\bar{e}_{a},\left[e_{a_{1}}, e_{a_{2}}\right]\right)} \otimes\left(H_{a_{1}}+H_{a_{2}}\right) \\
& -\bar{e}_{a} \otimes\left(e_{a},\left[\bar{e}_{a_{1}}, \bar{e}_{a_{2}}\right]\right) H_{a_{1}}-\bar{e}_{a} \otimes\left(e_{\alpha},\left[\bar{e}_{a_{1}}, \bar{e}_{a_{2}}\right]\right) \frac{\left(a_{2}, a_{2}\right)}{\left(a_{1}, a_{1}\right)} H_{a_{2}} .
\end{aligned}
$$

By Lemma 6.1.6 we have

$$
\left(\bar{e}_{a},\left[e_{a_{1}}, e_{a_{2}}\right]\right)\left(e_{a},\left[\bar{e}_{a_{2}}, \bar{e}_{\alpha_{1}}\right]\right)=1 / 2[(2 \delta, \alpha)-(\alpha, \alpha)]=-\left(\alpha_{1}, \alpha_{2}\right)
$$

(since $\alpha_{1}, \alpha_{2} \in \pi$ ). Therefore

$$
\begin{aligned}
& E x_{a}=\left[-1 / 2+\left(a_{1}, \alpha_{2}\right)\right]\left(\bar{e}_{a_{1}} \otimes \bar{e}_{a_{2}}+\frac{\left(a_{2}, a_{2}\right)}{\left(a_{1}, a_{1}\right)} \bar{e}_{a_{2}} \otimes \bar{e}_{a_{1}}\right) \\
& -1 / 2 \frac{\bar{e}_{a}}{\left(\bar{e}_{\alpha},\left[e_{a_{1}}, e_{a_{2}}\right]\right)} \otimes\left(\frac{\left(a_{2}, \alpha_{2}\right)}{\left(\alpha_{1}, \alpha_{1}\right)} H_{a_{1}}-H_{a_{2}}\right) \\
& +\left(\alpha_{1}, a_{2}\right) \frac{\bar{e}_{a}}{\left(\bar{e}_{a},\left[e_{a_{1}}, e_{a_{2}}\right]\right)} \otimes\left(\frac{\left(\alpha_{2}, a_{2}\right)}{\left(\alpha_{1}, a_{1}\right)} H_{a_{1}}-H_{\alpha_{2}}\right) \\
& =\left[-1 / 2+\left(\alpha_{1}, \alpha_{2}\right)\right] x_{a}=-c(-\alpha) x_{a}
\end{aligned}
$$

by (7.3.6). Hence $x_{\alpha}$ is harmonic; (see (7.2.9)). Q.E.D.

By $(7.1 .6)$

$$
H^{1}(n, g / n)=\sum_{\text {distinct weights } \xi}\left(\Lambda^{1} \bar{n} \otimes n^{\perp}\right)_{0}^{\xi} \quad \text { (direct sum) }
$$

where $\xi=-\alpha$ or $-\alpha-\beta, \alpha, \beta \in \Delta^{+}$. Therefore by Proposition 7.2.9, Corollary 7.3.10, Corollary 7.4.29, and Proposition 7.5.1 we can now state the main result of $\$ 7$ :

Theorem 7.5.3 Suppose $g$ is simple with rank $l$. Let 5 be a Cartan subalgebra of $g$ such that $\bar{G}=\bar{G}$ where conjugation on $g$ is defined by (2.2.2). Let $n=$ $\Sigma_{a \in \Delta^{+}} g_{a}$ be the nilpotent subalgebra of 9 generated by the positive root spaces. Then the first derived cohomology space $H^{1}(n, g / n)$ has dimension $l^{2}+l-1$ (over $\mathrm{C}$ ) and as an $\mathrm{G}$ module 


$$
H^{1}(n, g / n)=\sum_{a \in \pi} \mathrm{C} \bar{e}_{\alpha} \otimes \bar{e}_{a}+\sum_{a \in \pi} \xi^{\alpha}+\sum_{\substack{a \in \Delta^{+} \\ \text {sum of two simple roots }}} \mathrm{C} x_{\alpha} \text { (direct sum) }
$$

where $\pi$ is a system of simple roots and

$$
\xi^{a}=\left\{\bar{e}_{\alpha} \otimes H \mid \alpha(H)=0, H \in G\right\}
$$

(= byperplane defined by $a \in \pi)$ has dimension $l-1$

(ii) $x_{a}=\frac{\bar{e}_{a}}{\left(\bar{e}_{a},\left[e_{a_{1}}, e_{a_{2}}\right]\right)} \otimes\left(\frac{\left(a_{2}, a_{2}\right)}{\left(a_{1}, a_{1}\right)} H_{a_{1}}-H_{a_{2}}\right)+\bar{e}_{a_{1}} \otimes \bar{e}_{a_{2}}+\frac{\left(a_{2}, a_{2}\right)}{\left(a_{1}, a_{1}\right)} \bar{e}_{a_{2}} \otimes \bar{e}_{a_{1}}$ where $a=a_{1}+a_{2}, a_{1}, a_{2} \in \pi, a \in \Delta^{+}$, and where $H_{\beta}, \beta \in \Delta^{+}$, is given by (4.2.3). As in Theorem 5.3.12 $\left\{e_{\beta}\right\}_{\beta \epsilon \Delta^{+}},\left\{\bar{e}_{\beta}\right\}_{\beta \in \Delta^{-}}$are orthonormal bases of $n$ and $\bar{n}=\Sigma_{\beta \in \Delta^{+}} g_{-\beta}($ see $(2.2,1))$.

From the remarks immediately preceding the statement of Theorem 7.5.3, it suffices only to verify that $\operatorname{dim} H^{1}(n, g / n)=l^{2}+l-1$. For this we observe

Proposition 7.5.4 If $\mathrm{g}$ is simple with rank $l$ then the number of positive roots of level two (i.e. positive roots that are the sum of two simple roots) is $l-1$.

Proposition 7.5.4 follows by inspection. Hence

$$
\operatorname{dim} H^{1}(n, g / n)=l+l(l-1)+l-1=l^{2}+l-1 \text {. }
$$

8. Determination of the cohomology group $H^{1}\left(n, n^{\prime}\right)$.

8.1 In the final portion of this paper we shall discuss cohomology defined by the co-adjoint representation of $n$ on its dual $n^{\prime}$. This representation is denoted by $\theta^{\mathfrak{n}^{\prime}}$ where $\theta$ is the adjoint representation of $g$ on $g$. Recalling that there is a unitary map $\eta$ of $n^{\prime}$ onto $\bar{n}$ we shall regard $\theta^{n^{\prime}}$ as a representation of $n$ on $\bar{n}$. Then by Proposition 3.3.4

$$
\theta^{n^{\prime}}(x)=\left.P^{\bar{n}} \theta(x)\right|_{\bar{n}}, \quad x \in n,
$$

where $P^{\bar{n}}$ is the orthogonal projection of $g$ onto $\bar{n}$. The Laplace operator $L_{\theta^{n^{\prime}}}$ corresponding to $\theta^{\mathrm{n}^{\prime}}$ is given by Theorem 3.3.11:

$$
L_{\theta n^{\prime}}=\left(1 \otimes P^{\bar{n}}\right) L_{\left.\theta\right|_{n}}+E
$$

on $\Lambda \bar{n} \otimes \bar{n}$ where

$$
\begin{aligned}
E= & \sum_{\beta \in \Delta^{+}} \sum_{\gamma \in \Delta^{+}} \epsilon\left(\bar{e}_{\beta}\right) \iota\left(e_{\gamma}\right) \otimes P^{\bar{n}} \theta\left(\bar{e}_{\gamma}\right)\left(1-P^{\bar{\pi}}\right) \theta\left(e_{\beta}\right) \\
& +1 \otimes \sum_{\beta \in \Delta^{+}} P^{\bar{n}} \theta\left(\bar{e}_{\beta}\right)\left(P^{\bar{n}}-1\right) \theta\left(e_{\beta}\right) .
\end{aligned}
$$


Analogous to $(7.1 .6)$ we have

$$
H^{1}(\mathfrak{n}, \bar{n})=\sum_{\text {distinct } \xi}\left(\Lambda^{1} \bar{n} \otimes \bar{n}\right)_{0}^{\xi} \quad \text { (direct sum) }
$$

where $\xi$ is a weight $-\alpha-\beta, \alpha, \beta \in \Delta^{+}$, of the $\xi$-module $\Lambda^{1} \bar{n} \otimes \bar{n}$ and $\left(\Lambda^{1} \bar{n} \otimes \bar{n}\right)_{0}^{\xi}=\left\{x \in\left(\Lambda^{1} \bar{n} \otimes \bar{n}\right)^{\xi} \mid L_{\theta^{n^{\prime}}} x=0\right\}=$ space of $\theta^{n^{\prime}}$-harmonics. As in the case of $H^{1}(n, g / n)$ we must examine which weights contribute to cohomology.

One verifies immediately (using (8.1.1)) that $x \in L(n, \bar{n})$ (the space of linear maps from $n$ to $\bar{n})$ is a cocycle if and only if

$$
x\left(\left[y_{1}, y_{2}\right]\right)=P^{\bar{n}}\left(\left[x\left(y_{1}\right), y_{2}\right]+\left[y_{1}, x\left(y_{2}\right)\right]\right)
$$

for every $y_{1}, y_{2}$ in $n$; cf. (7.2.2).

8.2 Lemma 8.2.1 Let $\mu, \nu \in \Delta^{+}$. Then

$$
\begin{aligned}
E \bar{e}_{\mu} \otimes \bar{e}_{\nu}= & -1 / 2[(\lambda, \lambda+2 \delta)-(2 \delta, \nu)+(\nu, \nu)] \bar{e}_{\mu} \otimes \bar{e}_{\nu} \\
& +(\mu, \nu) \bar{e}_{\nu} \otimes \bar{e}_{\mu}+\sum_{\substack{\beta \in \Delta^{+} ; \beta-\nu \epsilon \Delta^{+} \\
\beta-(\mu+\nu) \epsilon \Delta^{-}}} \bar{e}_{\beta} \otimes\left[\bar{e}_{\mu},\left[e_{\beta}, \bar{e}_{\nu}\right]\right]
\end{aligned}
$$

where $E$ is the error term in (8.1.3); (cf. Lemma 7.2.4).

Proof. A direct (but uninspiring) calculation gives

$$
\begin{aligned}
E \bar{e}_{\mu} \otimes \bar{e}_{\nu}= & -(\nu, \nu) \bar{e}_{\mu} \otimes \bar{e}_{\nu}+(\mu, \nu) \bar{e}_{\nu} \otimes \bar{e}_{\mu} \\
& -\sum_{\beta \epsilon \Delta^{+} ; \beta-\nu \epsilon \Delta^{+}} \bar{e}_{\mu} \otimes\left[\bar{e}_{\beta},\left[e_{\beta}, \bar{e}_{\nu}\right]\right]+\sum_{\substack{\beta \in \Delta^{+} ; \beta-\nu \epsilon \Delta^{+} \\
\beta-(\mu+\nu) \epsilon \Delta^{-}}} \bar{e}_{\beta} \otimes\left[\bar{e}_{\mu},\left[e_{\beta}, \bar{e}_{\nu}\right]\right] .
\end{aligned}
$$

Now apply Lemma 6.1.1 (iii)。 Q.E.D.

Corollary 8.2.2 If $\mu \in \pi, \nu \in \Delta^{+}$, then

$$
E \bar{e}_{\mu} \otimes \bar{e}_{\nu}=-1 / 2[1-(2 \delta, \nu)+(\nu, \nu)] \bar{e}_{\mu} \otimes \bar{e}_{\nu}+(\mu, \nu) \bar{e}_{\nu} \otimes \bar{e}_{\mu}
$$

(see (7.3.5)).

Corollary 8.2.3 If $\mu, \nu \in \pi$, then

$$
E \bar{e}_{\mu} \otimes \bar{e}_{\nu}=-1 / \bar{i} \bar{e}_{\mu} \otimes \bar{e}_{\nu}+(\mu, \nu) \bar{e}_{\nu} \otimes \bar{e}_{\mu}
$$

Corollary 8.2.4 For every $\mu \in \pi, \bar{e}_{\mu} \otimes \bar{e}_{\mu}$ is $\theta^{n^{\prime}}$-harmonic.

Proof. Just as in (7.2.9) we have

$$
\begin{array}{ll}
L_{\theta^{n^{\prime}}}=0 & \text { if and only if } \\
E x=-c(\xi) x & \text { for } x \in(\Lambda \bar{n} \otimes \bar{n})^{\xi}
\end{array}
$$


where $E$ is given by (8.1.3). By (7.4.12) $c(-2 \mu)=1 / 2[1-2(\mu, \mu)]$ for $\mu \in \pi$. Hence $E \bar{e}_{\mu} \otimes \bar{e}_{\mu}=-c(-2 \mu) \bar{e}_{\mu} \otimes \bar{e}_{\mu}$ by Corollary 8.2.3。

In an entirely similar manner we obtain

Corollary 8.2.6 For every $\mu, \nu \in \pi, \bar{e}_{\mu} \otimes \bar{e}_{\nu}+\bar{e}_{\nu} \otimes \bar{e}_{\mu}$ is $\theta^{\mathfrak{n}^{\prime}}$-barmonic。

Corollary 8.2.7 If $\mu, \nu \in \pi$ such that $(\mu, \nu)=0$, then $\bar{e}_{\mu} \otimes \bar{e}_{\nu}$ is $\theta^{\mathfrak{n}^{\prime}}$-harmonic。

If $\alpha, \beta \in \Delta^{+}$then the general element $x$ in $\left(\Lambda^{1} \bar{n} \otimes \bar{n}\right)^{-\alpha-\beta}$ has the form

$$
x=\sum_{\mu, \nu \in \Delta^{+} ; \mu_{+}=\alpha_{+} \beta} c_{\mu \nu} \bar{e}_{\mu} \otimes \bar{e}_{\nu}, \quad c_{\mu \nu} \in \mathbf{C} .
$$

Lemma 8.2.9 Suppose $x$ given by $(8.2 .8)$ is harmonic. If $\mu \in \pi, \nu \in \Delta^{+}$such that $\mu+\nu=\alpha+\beta$ then $[-(2 \delta, \nu)+(\nu, \nu)+(\mu, \nu)] c_{\mu \nu}=(\mu, \nu) c_{\nu \mu} ; c f$. Lemma 7.4.11。In particular if $\nu \in \pi$ also then $(\mu, \nu) c_{\mu \nu}=(\mu, \nu) c_{\nu \mu}$ 。

Proof. By Lemma 8.2.1

$$
\begin{aligned}
E x= & -1 / 2 \sum_{\mu, \nu \in \Delta^{+} ; \mu+\nu=\alpha+\beta} c_{\mu \nu}[1-(2 \delta, \nu)+(\nu, \nu)] \bar{e}_{\mu} \otimes \bar{e}_{\nu} \\
& +\sum_{\mu, \nu \in \Delta^{+} ; \mu_{+} \nu=\alpha+\beta} c_{\mu \nu}(\mu, \nu) \bar{e}_{\nu} \otimes \bar{e}_{\mu} \\
& +\sum_{\mu, \nu \in \Delta^{+} ; \mu_{+} \nu=\alpha+\beta} \sum_{\substack{\beta \in \Delta^{+} ; \beta-\nu \in \Delta^{+} \\
\beta-(\mu+\nu) \in \Delta^{-}}} \bar{e}_{\beta} \otimes\left[\bar{e}_{\mu},\left[e_{\beta}, \bar{e}_{\nu}\right]\right] .
\end{aligned}
$$

Therefore if $\mu_{0} \in \pi, \nu_{0} \in \Delta^{+}$such that $\mu_{0}+\nu_{0}=\alpha+\beta$, then

$$
(E x)\left(e_{\mu_{0}}\right)=-1 / 2 c_{\mu_{0} \nu_{0}}\left[1-\left(2 \delta, \nu_{0}\right)+\left(\nu_{0}, \nu_{0}\right)\right] \bar{e}_{\nu_{0}}+c_{\nu_{0} \mu_{0}}\left(\mu_{0}, \nu_{0}\right) \bar{e}_{\nu_{0}} .
$$

On the other hand

$$
x\left(e_{\mu_{0}}\right)=c_{\mu_{0} \nu_{0}} \bar{e}_{\nu_{0}}
$$

and

$$
c(-\alpha-\beta)=c\left(-\mu_{0}-\nu_{0}\right)=1 / 2\left[1+\left(2 \delta, \nu_{0}\right)-2\left(\mu_{0}, \nu_{0}\right)-\left(\nu_{0}, \nu_{0}\right)\right]
$$

by (7.4.12). By (8.2.5) $(E x)\left(e_{\mu_{0}}\right)=-c\left(-\mu_{0}-\nu_{0}\right) x\left(e_{\mu_{0}}\right)$. Hence the lemma follows.

8.3 In the derivation of Theorem 7.5.3 crucial use of (7.4.17) was made. The continued study of $H^{1}(n, \bar{n})$ requires a similar relation. (7.4.17) is a direct consequence of the cocycle condition (7.4.4) (or (7.2.2)) of course. However if one applies the cocycle condition (8.1.5), proceeding in an analogous manner, one obtains the embarrassing result: $0=0$. The reason is that $P^{\bar{n}}$ annihilates $\mathscr{y}$. We shall indicate how this difficulty can be circumvented by bringing in a know- 
ledge of the cohomology group $H^{2}(n, \bar{h})$ where $\bar{b}$ is considered as a trivial $n$ module.

If $\nu$ is the trivial representation of $g$ on $V_{\nu}$ then by Theorem 2.4 .7 the Laplacian $L_{\left.\nu\right|_{\mathfrak{n}}}$ on $\Lambda \bar{n} \otimes V_{\nu}$ is given by

$$
2 L_{\left.\nu\right|_{\mathfrak{n}}}=-\sum_{i=1}^{l} \theta\left(\bar{H}_{i}\right) \theta\left(H_{i}\right) \otimes 1-\sum_{a \in \Delta^{+}} \theta\left(H_{\alpha}\right) \otimes 1 .
$$

The representation $\beta^{0}$ of $\mathfrak{G}$ on $\Lambda \bar{n} \otimes V_{\nu}$ is given by $\beta^{0}(H)=\theta(H) \otimes 1, H \in \mathfrak{K}$; see (4.3.6). If $\xi \in \mathfrak{G}^{\prime}$ then

$$
L_{\left.\nu\right|_{\mathfrak{n}}}=-1 / 2[(\xi, \xi)+(\xi, 2 \delta)]
$$

on $\left(\Lambda \bar{n} \otimes V_{\nu}\right)^{\xi}$; cf. The orem 4.3.8. $\beta^{0}$ and $L$ clearly commute since $\mathscr{G}$ is abelian. It is easy to see that

$$
\Lambda^{2} \bar{n} \otimes V_{\nu}=\sum_{a_{1}, a_{2} \epsilon \Delta^{+} ; a_{1}<a_{2}}\left(\Lambda^{2} \bar{n} \otimes V_{\nu}\right)^{-a_{1}-a_{2}} \quad \text { (direct sum) }
$$

where $x \in\left(\Lambda^{2} \bar{n} \otimes V_{\nu}\right)^{-a_{1}-a_{2}}$ if and only if

$$
x=\sum_{\substack{\beta, \gamma \in \Delta^{+} ; \beta<\gamma \\ \beta+\gamma=a_{1}+\alpha_{2}}} c_{\beta \gamma}\left(\bar{e}_{\beta} \wedge \bar{e}_{\gamma}\right) \otimes v, \quad v \in V_{\nu} \cdot
$$

Since the weight spaces are $L_{\left.\nu\right|_{n}}$ invariant (because $\beta^{0}$ and $L_{\left.\nu\right|_{\mathfrak{n}}}$ commute)

$$
H^{2}\left(m, V_{\nu}\right)=\sum_{\substack{a_{1}, a_{2} \epsilon \Delta^{+} ; a_{1}<a_{2} \\\left(a_{1}+a_{2}, a_{1}+a_{2}\right)=\left(a_{1}+a_{2}, 2 \delta\right)}}\left(\Lambda^{2} \bar{r} \otimes V_{\nu}\right)^{-a_{1}-a_{2}}
$$

by (8.3.2). Also because $L_{\left.\nu\right|_{\mathfrak{n}}}$ is positive definite (8.3.2) implies

Proposition 8.3.6 For $a_{1}, a_{2} \in \Delta^{+}, \alpha_{1} \neq a_{2}$,

$$
\left(a_{1}+a_{2}, a_{1}+a_{2}\right) \leq\left(a_{1}+a_{2}, 2 \delta\right) \text {. }
$$

Proposition 8.3.7 If $a_{1}, a_{2} \in \Delta^{+}$such that $\left(a_{1}+a_{2}, a_{1}+a_{2}\right)=\left(a_{1}+a_{2}, 2 \delta\right)$ then $\alpha_{1}+a_{2}$ is not a root.

Proof. We may assume of course that $\alpha_{1} \neq a_{2}$, say $a_{1}<a_{2}$. Let $v \in V_{\nu}, v \neq 0$ be arbitrary. By (8.3.5) $\left(\bar{e}_{a_{1}} \wedge \bar{e}_{a_{2}}\right) \otimes v$ is harmonic. In particular $0=$ $c_{\nu}^{*}\left(\left(\bar{e}_{a_{1}} \wedge \bar{e}_{a_{2}}\right) \otimes v\right)$ by (2.5.6). However $c_{\nu}^{*}=c^{*} \otimes 1=\partial \otimes 1$ by (2.5.4) since $c_{2}=0$. Therefore by $(2.5 .1)$

$$
0=\left[\bar{e}_{a_{1}}, \bar{e}_{a_{2}}\right] \otimes v \text { implies } 0=\left[\bar{e}_{\alpha_{1}}, \bar{e}_{a_{2}}\right]
$$

implies $a_{1}+\alpha_{2}$ is not a root. 
We can now prove Proposition 4.2.13:

Corollary 8.3.8 Let $\beta \in \Delta^{+}$. Then $\beta \in \pi$ if and only if $(2 \delta, \beta)=(\beta, \beta)$.

Proof. If $\beta \in \pi$ then $(2 \delta, \beta)=(\beta, \beta)$. This is easy to check; see proof of Lemma 2, [5, p. 248]. A denial of the converse would be a denial of Proposition 8.3.7. Q.E.D.

8.4 Consider the short exact sequence of $n$ modules:

$$
0 \rightarrow \overline{5} \rightarrow \overline{5}+\bar{n} \rightarrow \bar{n} \rightarrow 0 .
$$

This sequence gives rise to a long exact sequence of cohomology groups. A portion of the latter sequence is

$$
\cdots \rightarrow H^{1}(n, \bar{\zeta}+\bar{n}) \rightarrow H^{1}(n, \bar{n}) \rightarrow H^{2}(n, \bar{h}) \rightarrow H^{2}(n, \bar{G}+n) \rightarrow \cdots
$$

For later applications it is useful to display the "connecting" Bockstein homomorphism $H^{1}(n, \bar{n}) \rightarrow H^{2}(n, \bar{G})$ explicitly. The orthogonal projection $P=P^{g / n}$ of $\bar{G}+\bar{n}$ onto $\bar{n}$ induces a homomorphism $P: H^{1}(n, \bar{G}+\bar{n}) \rightarrow H^{1}(n, \bar{n})$. On the cocycle level $P$ is given by

$$
(P x)(y)=P x(y)
$$

where $x \in L(n, \bar{G}+\bar{n})$ satisfies (7.2.2) and $y \in n$ 。

The basis for constructing the connecting homomorphism of $H^{1}(n, \bar{n})$ into $H^{2}(n, G)$ is given by

Lemma 8.4.2 Let $\omega=P^{b}$ be the orthogonal projection of $g$ onto 5 . Given $x \in L(n, \bar{n})$ define $\omega x \in \Lambda^{2}(n, \bar{\zeta})($ see $\$ 2.4)$ by

$$
(\omega x)\left(y_{1}, y_{2}\right)=\omega\left(\left[x\left(y_{1}\right), y_{2}\right]+\left[y_{1}, x\left(y_{2}\right)\right]\right)
$$

where $y_{1}, y_{2} \in n_{\text {. If } x}$ is a 1-cocycle, i.e. $x$ satisfies (8.1.5), then $\omega x$ is a 2 cocycle; i.e. for every $y_{1}, y_{2}, y_{3} \in \mathrm{n}$

$$
(\omega x)\left(\left[y_{1}, y_{2}\right], y_{3}\right)=(\omega x)\left(\left[y_{1}, y_{3}\right], y_{2}\right)-(\omega x)\left(\left[y_{2}, y_{3}\right], y_{1}\right) ;
$$

(see (2.4.1)).

Proof. First we observe that

$$
\omega\left[P^{\bar{n}} z, y\right]=\omega[z, y]
$$

for $z \in g, y \in n$. By (8.1.5), (8.4.3) 


$$
\begin{aligned}
-(\omega x) & \left(\left[y_{1}, y_{2}\right], y_{3}\right)+(\omega x)\left(\left[y_{1}, y_{3}\right], y_{2}\right)-(\omega x)\left(\left[y_{2}, y_{3}\right], y_{1}\right) \\
= & -\omega\left(\left[x\left(\left[y_{1}, y_{2}\right]\right), y_{3}\right]+\left[\left[y_{1}, y_{2}\right], x\left(y_{3}\right)\right]\right) \\
& +\omega\left(\left[x\left(\left[y_{1}, y_{3}\right]\right), y_{2}\right]+\left[\left[y_{1}, y_{3}\right], x\left(y_{2}\right)\right]\right) \\
& -\omega\left(\left[x\left(\left[y_{2}, y_{3}\right]\right), y_{1}\right]+\left[\left[y_{2}, y_{3}\right], x\left(y_{1}\right)\right]\right) \\
= & -\omega\left(\left[P^{\bar{n}}\left(\left[x\left(y_{1}\right), y_{2}\right]+\left[y_{1}, x\left(y_{2}\right)\right]\right), y_{3}\right]\right)-\omega\left[\left[y_{1}, y_{2}\right], x\left(y_{3}\right)\right] \\
& +\omega\left(\left[P^{\bar{n}}\left(\left[x\left(y_{1}\right), y_{3}\right]+\left[y_{1}, x\left(y_{3}\right)\right]\right), y_{2}\right]\right)+\omega\left[\left[y_{1}, y_{3}\right], x\left(y_{2}\right)\right] \\
& -\omega\left(\left[P^{\bar{n}}\left(\left[x\left(y_{2}\right), y_{3}\right]+\left[y_{2}, x\left(y_{3}\right)\right]\right), y_{1}\right]\right)-\omega\left[\left[y_{2}, y_{3}\right], x\left(y_{1}\right)\right] \\
= & -\omega\left[\left[x\left(y_{1}\right), y_{2}\right], y_{3}\right]-\omega\left[\left[y_{1}, x\left(y_{2}\right)\right], y_{3}\right]-\omega\left[\left[y_{1}, y_{2}\right], x\left(y_{3}\right)\right] \\
& +\omega\left[\left[x\left(y_{1}\right), y_{3}\right], y_{2}\right]+\omega\left[\left[y_{1}, x\left(y_{3}\right)\right], y_{2}\right]+\omega\left[\left[y_{1}, y_{3}\right], x\left(y_{2}\right)\right] \\
& -\omega\left[\left[x\left(y_{2}\right), y_{3}\right], y_{1}\right]-\omega\left[\left[y_{2}, x\left(y_{3}\right)\right], y_{1}\right]-\omega\left[\left[y_{2}, y_{3}\right], x\left(y_{1}\right)\right] \quad \text { (by (8.4.5)) } \\
= & 0
\end{aligned}
$$

by three applications of the Jacobi identity, for every $y_{1}, y_{2}, y_{3} \in n$. Q.E.D.

It is also true that $\omega x$ is a coboundary if $x$ is a coboundary. Therefore $\omega$ defines a homomorphis.m $\omega: H^{1}(n, \bar{n}) \rightarrow H^{2}(n, \bar{h})$. Moreover

$$
H^{1}(n, \bar{\zeta}+\bar{n}) \stackrel{P}{\rightarrow} H^{1}(n, \bar{n}) \stackrel{\omega}{\rightarrow} H^{2}(n, \bar{\zeta})
$$

is exact. Now in view of Corollary 2.1.4 (a consequence of the Hodge decomposition) every cocycle is cohomologous to a unique harmonic cocycle. Thus $\omega x$, for $x$ a 1 -cocycle, differs from a unique $\theta^{n^{\prime}}$-harmonic by a coboundary $\delta_{1} f_{x}, f_{x} \epsilon$ $L(n, \mathfrak{G})$. By $(2.4 .1)$

$$
\left(\delta_{1} f_{x}\right)\left(y_{1}, y_{2}\right)=-f_{x}\left(\left[y_{1}, y_{2}\right]\right)
$$

for $y_{1}, y_{2}$ in $n$. Hence for a unique $x_{0}$ in $H^{2}(n, 5)$

$$
(\omega x)\left(y_{1}, y_{2}\right)=-f_{x}\left(\left[y_{1}, y_{2}\right]\right)+x_{0}\left(y_{1} \wedge y_{2}\right)
$$

for every $y_{1}, y_{2}$ in $n$. On the other hand by (8.3.4) and (8.3.5) we can write

$$
x_{0}=\sum_{\begin{array}{c}
a_{1}, a_{2} \in \Delta^{+} ; a_{1}<a_{2} \\
\left|a_{1}+a_{2}\right|^{2}=\left(a_{1}+a_{2}, 2 \delta\right)
\end{array}}
$$

where

$$
x_{a_{1, a_{2}}}=\sum_{\substack{\beta, \gamma \in \Delta^{+} \\ \beta+\gamma=a_{1}+a_{2} ; \beta<\gamma}} d_{\beta, \gamma^{\prime}}\left(\bar{e}_{\beta} \wedge \bar{e}_{\gamma}\right) \otimes H_{a_{1}, a_{2}}
$$

with $H_{\dot{a}_{1}, a_{2}} \in \mathscr{G}$. Now let $\alpha_{0}, \beta_{0} \in \Delta^{+}$and suppose the cocycle $x \in$ 
$\left(\Lambda^{1} \bar{n} \otimes \bar{n}\right)^{-a_{0}-\beta_{0}}$ so that by (8.2.8)

$$
x=\sum_{\mu, \nu \in \Delta^{+} ; \mu+\nu=\alpha_{0}+\beta_{0}} c_{\mu \nu} \bar{e}_{\mu} \otimes \bar{e}_{\nu^{\circ}}
$$

Given $\mu, \nu \in \Delta^{+}$such that $\mu+\nu=\alpha_{0}+\beta_{0}$ take $y_{1}=e_{\mu}, y_{2}=e_{\nu}$ in (8.4.7). Then using (8.4.3) we get

$$
-c_{\mu \nu} H_{\nu}+c_{\nu \mu} H_{\mu}=-f_{x}\left(\left[e_{\mu}, e_{\nu}\right]\right)+x_{0}\left(e_{\mu} \wedge e_{\nu}\right) ;
$$

cf. (7.4.17). This is the relation we desire with regard to the remarks made at the beginning of $\$ 8.3$.

Lemma 8.4.12 Suppose $\mu, \nu, \mu+\nu \in \Delta^{+}$. Then $x_{0}\left(e_{\mu} \wedge e_{\nu}\right)=0$ for $x_{0}$ given by (8.4.8).

Proof. By (8.4.9)

$$
x_{a_{1}, a_{2}}\left(e_{\mu} \wedge e_{\nu}\right)=\sum_{\substack{\beta, \gamma \in \Delta^{+} \\ \beta+\gamma=a_{1}+a_{2} ; \beta<\gamma}} d_{\beta, \gamma}\left(\bar{e}_{\beta} \wedge \bar{e}_{\gamma}, e_{\mu} \wedge e_{\nu}\right) H_{a_{1}, a_{2}}
$$

$=0$ unless $\beta=\mu, \gamma=\nu$ or $\beta=\nu, \gamma=\mu$; see (2.2.5). The latter two cases require $\mu+\nu=\beta+\gamma=\alpha_{1}+\alpha_{2}$ so that $|\mu+\nu|^{2}=\left|\alpha_{1}+\alpha_{2}\right|^{2}=\left(\alpha_{1}+\alpha_{2}, 2 \delta\right)($ see $(8.4 .8))=$ $(\mu+\nu, 2 \delta)$ which contradicts Proposition 8.3.7 since $\mu+\nu$ is a root. Hence $x_{a_{1}, a_{2}}\left(e_{\mu} \wedge e_{\nu}\right)=0$ implies $x_{0}\left(e_{\mu} \wedge e_{\nu}\right)=0$.

Corollary 8.4.13 Suppose that $x$ given by (8.4.10) is a cocycle and suppose $\alpha_{0}+\beta_{0}$ is a root. Then for $\mu, \nu \in \Delta^{+}$such that $\mu+\nu=\alpha_{0}+\beta_{0}$ we have

$$
-c_{\mu \nu} H_{\nu}+c_{\nu \mu} H_{\mu}=\left(\bar{e}_{a_{0}+\beta_{0}},\left[e_{\mu}, e_{\nu}\right]\right)\left(-f_{x}\left(e_{\alpha_{0+\beta_{0}}}\right)\right) \text {. }
$$

Proof. $\left[e_{\mu}, e_{\nu}\right]=\left(\bar{e}_{a_{0}+\beta_{0}},\left[e_{\mu}, e_{\nu}\right]\right) e_{a_{0}+\beta_{0}}$. Hence Lemma 8.4 .12 and (8.4.11) imply Corollary 8.4.13.

8.5 Proposition 8.5.1 Suppose $\alpha_{0}, \beta_{0}, \alpha_{0}+\beta_{0} \in \Delta^{+}$. Then $\left(\Lambda^{1} \bar{n} \otimes \bar{n}\right)_{0}^{-\alpha_{0}-\beta_{0}}=$ $\{0\}$ unless $\alpha_{0}, \beta_{0} \in \pi$ (see Proposition 8.5.6).

Proof. First we assume that $g$ is nonsimply laced. Let $\gamma=\alpha_{0}+\beta_{0} \in \Delta^{+}$. Suppose that $x$ given by (8.4.10) is harmonic and assume that $\alpha_{0}$ or $\beta_{0}$ is nonsimple. By Scholium 7.4.20 $\gamma=\gamma_{0}+\beta_{1}+\beta_{2}$ where $\gamma_{0} \in \pi, \beta_{1}, \beta_{2}, \gamma_{0}+\beta_{1}$, $\beta_{1}+\beta_{2} \in \Delta^{+}$but $\gamma_{0}+\beta_{2}$ is not a root. By Lemma 7.4.5

$$
c_{\gamma_{0} \beta}=-c_{\beta \gamma_{0}}
$$

where $\beta=\beta_{1}+\beta_{2} \in \Delta^{+}$. By Lemma 8.2.9 
$\left[-(2 \delta, \beta)+(\beta, \beta)+\left(\gamma_{0}, \beta\right)\left(c_{\gamma_{0} \beta}\right)\right]_{c_{\gamma_{0} \beta}}=\left(\gamma_{0}, \beta\right) c_{\beta \gamma_{0}}$

$$
=-\left(\gamma_{0} \beta\right) c_{\gamma_{0} \beta} \text { by (8.5.1). }
$$

If $c \gamma_{0} \beta \neq 0$ then $(2 \delta, \gamma)=\left(2 \delta, \gamma_{0}\right)+(2 \delta, \beta)=\left(\gamma_{0}, \gamma_{0}\right)+(\beta, \beta)+2\left(\gamma_{0}, \beta\right)=(\gamma, \gamma)$ so $\gamma \in \pi$ by Corollary 8.3.8. This is a contradiction; hence $c_{\gamma_{0} \beta}=0$. By $(8.5 .1)$ and Corollary 8.4.13 $f_{x}\left(e_{\gamma}\right)=0$. Applying Corollary 8.4.13 again we get $c_{\mu \nu} \nu=$ $c_{\nu \mu} \mu$ for $\mu, \nu \in \Delta^{+}$such that $\mu+\nu=\alpha_{0}+\beta_{0}=\gamma \cdot \gamma=$ root implies $\mu \neq \nu$ and hence $c_{\mu \nu}=c_{\nu \mu}=0$ implies $x=0$. This proves the proposition for the nonsimply laced case. Now assume $g$ is simply laced; i.e. all roots of $g$ have the same length. Then for $\mu, \nu \in \Delta^{+}$such that $\mu+\nu=\gamma$

$$
(\mu, \nu)=(\gamma, \gamma) / 2
$$

by (7.4.26). Let $H=-f_{x}\left(e_{\gamma}\right) \in$ G. Then by Corollary 8.4.13

(8.5.4) $\gamma(H)\left(\bar{e}_{\gamma},\left[e_{\mu}, e_{\nu}\right]\right)=-c_{\mu \nu}(\gamma, \nu)+c_{\nu \mu}(\gamma, \mu)=\left(c_{\nu \mu}-c_{\mu \nu}\right)(\gamma, \gamma) / 2$

by (8.5.3) and the fact that $g$ is simply laced; cf. (7.4.27). Moreover by (3.3.9) and (7.4.23)

$$
\sum_{\mu, \nu \in \Delta^{+} ; \mu+\nu=\gamma} c_{\mu \nu}\left[\bar{e}_{\mu}, \bar{e}_{\nu}\right]=0 ;
$$

cf. (7.4.24). Thus we have all the ingredients necessary to imitate the proof of Proposition 7.4.25. Indeed by arguments entirely similar to those given there we see that $\gamma(H)=0$ and hence

$$
c_{\nu \mu}=c_{\mu \nu}
$$

by (8.5.4) for all $\mu, \nu \in \Delta^{+}$such that $\mu+\nu=\gamma$. Next note that $c_{\mu \nu}=0$ for $\mu \in \pi$, $\nu \notin \pi$. Indeed by Lemma 8.2.9 and (8.5.5)

$$
[-(2 \delta, \nu)+(\nu, \nu)+(\mu, \nu)]_{\mu \nu}=(\mu, \nu) c_{\nu \mu}=(\mu, \nu) c_{\mu \nu}
$$

so that $(2 \delta, \nu)=(\nu, \nu)$ if $c_{\mu \nu} \neq 0$; cf. Corollary 8.3.8. Therefore by essentially the argument which follows (7.4.28) we have $x=0$ unless both $\alpha_{0}, \beta_{0} \in \pi$.

Proposition 8.5.6 Suppose $\alpha_{0}, \beta_{0} \in \pi$ and $\alpha_{0}+\beta_{0} \in \Delta^{+}$. Then $\left(\Lambda^{1} \bar{n} \otimes \bar{n}\right)_{0}^{-\alpha_{0}-\beta_{0}}=\mathbf{C}\left(\bar{e}_{\alpha_{0}} \otimes \bar{e}_{\beta_{0}}+\bar{e}_{\beta_{0}} \otimes \bar{e}_{\alpha_{0}}\right)$.

Proof. Let $x=a \bar{e}_{a_{0}} \otimes \bar{e}_{\beta_{0}}+b \bar{e}_{\beta_{0}} \otimes \bar{e}_{a_{0}}$ be an arbitrary vector in $\left(\Lambda^{1} \bar{n} \otimes \bar{n}\right)^{-\alpha_{0}-\beta_{0}}$. If $x$ is harmonic, then $\left(\alpha_{0}, \beta_{0}\right) a=\left(\alpha_{0}, \beta_{0}\right) b$ by Lemma 8.2.9. Since $\alpha_{0}, \beta_{0} \in \pi$ and $\alpha_{0}+\beta_{0}$ is a root, $\left(\alpha_{0}, \beta_{0}\right) \neq 0$. Hence $a=b$ implies

$$
\left(\Lambda^{1} \bar{n} \otimes \bar{n}\right)_{0}^{-a_{0}-\beta_{0}} \subset \mathbf{C}\left(\bar{e}_{a_{0}} \otimes \bar{e}_{\beta_{0}}+\bar{e}_{\beta_{0}} \otimes \bar{e}_{a_{0}}\right) \text {. }
$$

By Corollary 8.2.7 we have equality. Q.E.D. 
Having now described all harmonic weight vectors for weights $-\alpha-\beta$, where $\alpha, \beta, \alpha+\beta \epsilon \Delta^{+}$we turn to consider the weights $-\alpha-\beta, \alpha, \beta \in \Delta^{+}$, such that $\alpha+\beta$ is not a root. Such weights fall into two classes according to equality or inequality of the numbers $|a+\beta|^{2},(\alpha+\beta, 2 \delta)$.

8.6 Proposition 8.6.1 Suppose $a_{0}, \beta_{0} \in \Delta^{+}$such that $a_{0}+\beta_{0}$ is not a root and such that $\left|a_{0}+\beta_{0}\right|^{2} \neq\left(\alpha_{0}+\beta_{0}, 2 \delta\right)$. Then

$$
\left(\Lambda^{1} \bar{n} \otimes \bar{n}\right)_{0}^{-a_{0}-\beta_{0}}=\{0\}
$$

unless $\alpha_{0}+\beta_{0}$ is of the form $\alpha_{0}+\beta_{0}=2 \gamma_{0}, \gamma_{0} \in \Delta^{+}$. On the other hand

$$
\left(\Lambda^{1} \bar{n} \otimes \bar{n}\right)_{0}^{-2 \gamma_{0}}= \begin{cases}\{0\} & \text { if } \gamma_{0} \notin \pi, \\ \mathbf{C}^{-} \bar{e}_{\gamma_{0}} \otimes \bar{e}_{\gamma_{0}} & \text { if } \gamma_{0} \in \pi,\end{cases}
$$

for any $\gamma_{0} \in \Delta^{+}(c f$. Proposition 7.2.7).

Proof. By the argument given in the proof of Lemma 8.4.12 $x_{0}\left(e_{\mu} \wedge e_{\nu}\right)=0$ for $\mu, \nu \in \Delta^{+}$such that $\mu+\nu=a_{0}+\beta_{0}$ where $x$ is a cocycle in $\left(\Lambda^{1} \bar{n} \otimes \bar{n}\right)^{-a_{0}-\beta_{0}}$ given by (8.4.10). Also $\left[e_{\mu}, e_{\nu}\right]=0$ since $\mu+\nu$ is not a root. Therefore by (8.4.11)

$$
-c_{\mu \nu} H_{\nu}+c_{\nu \mu} H_{\mu}=0
$$

or $c_{\mu \nu} \nu=c_{\nu \mu} \mu$. If $\alpha_{0}+\beta_{0}$ is not of the form $2 \gamma, \gamma \in \Delta^{+}$, then $\mu \neq \nu$ implies $c_{\mu \nu}=c_{\nu \mu}=0$ implies $x=0$. If $\alpha_{0}+\beta_{0}=2 \gamma_{0}$ for some $\gamma_{0} \epsilon \Delta^{+}$, then we have just shown that

$$
\left(\Lambda^{1} \bar{n} \otimes \bar{n}\right)^{-2 \gamma_{0}} \subset \mathrm{C}_{\gamma_{0}} \otimes \bar{e}_{\gamma_{0}} .
$$

If $\gamma_{0} \in \pi$, then we have equality by Corollary 8.2.4. Conversely if $x=\bar{e}_{\gamma_{0}} \otimes$ $\bar{e}_{\gamma_{0}}$ is harmonic then $x$ is a cocycle in particular so $\gamma_{0} \in \pi$ by the argument in the proof of Proposition 7.2.3. Q.E.D.

Note that indeed $\left|2 \gamma_{0}\right|^{2} \neq\left(2 \gamma_{0}, 2 \delta\right)$ for $\gamma_{0} \in \pi$.

8.7 The contribution to cohomology of weights of the form $-\alpha-\beta, \alpha, \beta \in \Delta^{+}$, where $|\alpha+\beta|^{2}=(\alpha+\beta, 2 \delta)$ must be considered, finally, to complete our analysis. First we shall characterize such weights:

Lemma 8.7.1 Let $\gamma_{1}, \gamma_{2}$ be distinct simple roots and let $S_{\gamma_{2}}$ be the simple Weyl reflection defined by $\gamma_{2}$. Then $\gamma_{2}, s_{\gamma_{2}} \gamma_{1}$ are positive roots such that

$$
\left|\gamma_{2}+s_{\gamma_{2}} \gamma_{1}\right|^{2}=\left(\gamma_{2}+S_{\gamma_{2}} \gamma_{1}, 2 \delta\right) \text {. }
$$

Conversely if $\alpha, \beta$ are positive roots that satisfy (8.7.2) then there exist distinct 
simple roots $\gamma_{1}, \gamma_{2}$ such that the sets $\{a, \beta\},\left\{\gamma_{2}, s_{\gamma_{2}} \gamma_{1}\right\}$ coincide. Moreover if $\gamma_{1}, \gamma_{2}, \mu_{1}, \mu_{2}$ are simple roots such that $\gamma_{2}+S_{\gamma_{2}} \gamma_{1}=\mu_{2}+S_{\mu_{2}} \mu_{1}, \gamma_{1} \neq \gamma_{2}$, $\mu_{1} \neq \mu_{2}$ then the sets $\left\{\gamma_{2}, s_{\gamma_{2}} \gamma_{1}\right\},\left\{\mu_{2}, s_{\mu_{2}} \mu_{1}\right\}$ coincide.

Proof. If $\gamma_{1}, \gamma_{2} \in \pi$ then $\left(\gamma_{2}+s_{\gamma_{2}} \gamma_{1}, 2 \delta\right)=\left|\gamma_{2}+s_{\gamma_{2}} \gamma_{1}\right|^{2}$ by (4.2.11), Proposition 4.2.13, and the fact that $S_{\gamma_{2}}$ is orthogonal. Conversely let $\alpha, \beta \in \Delta^{+}$ such that $|a+\beta|^{2}=(\alpha+\beta, 2 \delta)$. Then

$$
|\delta|^{2}=|\delta-a-\beta|^{2}
$$

We shall outline an argument which, up to a point, is quite similar to Cartier's argument given in the proof of Lemma 5.2.4. Put $\Phi=\{\alpha, \beta\} \subset \Delta^{+}, \xi=$ $-\alpha-\beta$. Then $\xi=-\langle\Phi\rangle$ so that

$$
\delta+\frac{\xi}{s}=-1 / 2\left\langle\psi_{\phi}\right\rangle
$$

by (5.2.7) and for every $S$ in the Weyl group $W$

$$
s(\delta+\xi)=\delta-\left\langle\Phi_{S}\right\rangle
$$

where $\Phi_{S}=S \psi_{\phi} \cap \Delta^{+}$; cf. (5.2.13). If $\left\langle\Phi_{S}\right\rangle=\Sigma_{\gamma \in \Delta^{+}}{ }^{n} \gamma \gamma$ where the $n \gamma$ are nonnegative integers, then choosing $S \in W$ such that $(S(\delta+\xi), \gamma) \geq 0$ for every $\gamma$ in $\Delta^{+}$we have each $n_{\gamma}=0$ by $(8,7.3)$ and (8.7.5). Hence $\Phi_{S}=\varnothing$ or

$$
S^{-1} \Delta^{-} \cap \Delta^{+}=\Phi=\{\alpha, \beta\} \text {. }
$$

Thus $S$ has length two:

$$
S=S_{\gamma_{1}} S_{\gamma_{2}}, \quad \gamma_{1}, \gamma_{2} \in \pi
$$

see [4]. (8.7.6) implies $S \neq 1$; i.e. $\gamma_{1} \neq \gamma_{2}$. Since $s_{\gamma_{2}} \gamma_{1}=s_{\gamma_{2}} s_{\gamma_{1}}\left(-\gamma_{1}\right)$, we have $S_{\gamma_{2}} \gamma_{1} \in S^{-1} \Delta^{-} \cap \Delta^{+}$. Similarly $\gamma_{2}=S_{\gamma_{2}} S_{\gamma_{1}}\left(-S_{\gamma_{1}} \gamma_{2}\right) \in S^{-1} \Delta^{-} \cap \Delta^{+}$ so that

$$
\left\{s_{\gamma_{2}} \gamma_{1}, \gamma_{2}\right\}=\{\alpha, \beta\}
$$

by (8.7.6). Suppose $\gamma_{1}, \gamma_{2}, \mu_{1}, \mu_{2} \epsilon \pi$ with $\gamma_{2}+S_{\gamma_{2}} \gamma_{1}=\mu_{2}+S_{\mu_{2}} \mu_{1}, \gamma_{1} \neq \gamma_{2}$, $\mu_{1} \neq \mu_{2}$. Then

or

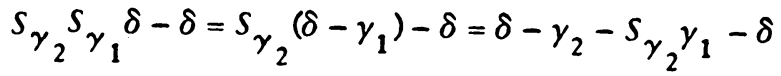

$$
\begin{aligned}
& =-\mu_{2}-S_{\mu_{2}} \mu_{1}=S_{\mu_{2}} S_{\mu_{1}} \delta-\delta
\end{aligned}
$$

$$
s_{\gamma_{2}} s_{\gamma_{1}} \delta=S_{\gamma_{2}} s_{\gamma_{1}} \delta \text {. }
$$

Since $\delta$ is regular $S_{\gamma_{2}} S_{\gamma_{1}}=S_{\mu_{2}} S_{\mu_{1}}$ (the Weyl group acts simply transitively on 
the systems of simple roots). By (8.7 6) and (8.7.7) we have

$$
\begin{aligned}
\left\{\gamma_{2}, S_{\gamma_{2}} \gamma_{1}\right\} & =S_{\gamma_{2}} S_{\gamma_{1}} \Delta^{-} \cap \Delta^{+} \\
& =S_{\mu_{2}} S_{\mu_{1}} \Delta^{-} \cap \Delta^{+}=\left\{\mu_{2}, S_{\mu_{2}} \mu_{1}\right\} \text { Q.E.D. }
\end{aligned}
$$

It follows that the weights in (8.3.5) have multiplicity one. This is known of course by Kostant's work. We also have the following

Corollary 8.7.9 If $\gamma_{1}, \gamma_{2} \in \pi$ are distinct then $\gamma_{2}+S_{\gamma_{2}} \gamma_{1}$ is not a root; see Proposition 8.3.7.

Proposition 8.7.10 If $\alpha, \beta$ are simple roots such that $|\alpha+\beta|^{2}=(\alpha+\beta, 2 \delta)$ then $\left(\Lambda^{1} \bar{n} \otimes \bar{n}\right)_{0}^{-\alpha-\beta}=\mathbf{C} \bar{e}_{\alpha} \otimes \bar{e}_{\beta}+\mathbf{C} \bar{e}_{\beta} \otimes \bar{e}_{\alpha}$.

Proof. Since $\alpha, \beta \in \pi,|\alpha+\beta|^{2}=(\alpha+\beta, 2 \delta)$ if and only if $(\alpha, \beta)=0$. Therefore Proposition 8.7.10 follows from (8.2.8) and Corollary 8.2.7.

Proposition 8.7.11 Let $\gamma_{1}, \gamma_{2}$ be distinct simple roots. Assume $s_{\gamma_{2}} \gamma_{1}$ is not a simple root. Then

$$
x_{\gamma_{1}, \gamma_{2}}=\bar{e}_{\gamma_{2}} \otimes \bar{e}_{s_{\gamma_{2}} \gamma_{1}}-\bar{e}_{s_{\gamma_{2}} \gamma_{1}} \otimes \bar{e}_{\gamma_{2}}
$$

is a barmonic vector and in fact

$$
\left(\Lambda^{1} \bar{n} \otimes \bar{n}\right)_{0}^{-\gamma_{2}-s_{\gamma_{2}} \gamma_{1}}=\mathrm{C} x_{\gamma_{1}, \gamma_{2}}
$$

Proof. Let

$$
x \in\left(\Lambda^{1} \bar{n} \otimes \bar{n}\right)^{-\gamma_{2}-s_{\gamma}}{ }_{2}^{\gamma_{1}}
$$

be arbitrary. By (8.2.8) and Lemma 8.7.1

$$
x=a \bar{e} \gamma_{2} \otimes \bar{e}_{s_{\gamma_{2}} \gamma_{1}}+b \bar{e} s_{\gamma_{2} \gamma_{1}} \otimes \bar{e} \gamma_{2}
$$

$a, b \in \mathrm{C}$. Now assume $x$ is harmonic. Then by Lemma 8.2.9

$$
\left[-\left(2 \delta, s_{\gamma_{2}} \gamma_{1}\right)+\left(s_{\gamma_{2}} \gamma_{1}, s_{\gamma_{2}} \gamma_{1}\right)+\left(\gamma_{2}, s_{\gamma_{2}} \gamma_{1}\right)\right] a=\left(\gamma_{2}, s_{\gamma_{2}} \gamma_{1}\right) b
$$

or since $S_{\gamma_{2}}$ is orthogonal

$$
\left(\gamma_{2}, \gamma_{1}\right) a=-\left(\gamma_{2}, \gamma_{1}\right) b
$$

Now $S_{\gamma_{2}} \gamma_{1}=\gamma_{1}-\left(2\left(\gamma_{2}, \gamma_{1}\right) /\left(\gamma_{2}, \gamma_{2}\right)\right) \gamma_{2}$; so because $s_{\gamma_{2}} \gamma_{1}$ is nonsimple we must have $\left(\gamma_{2}, \gamma_{1}\right) \neq 0$. Therefore $a=-b$ implies $x$ equals $x_{\gamma_{1}, \gamma_{2}}$; see (8.7.12). The proof is then reduced to showing that $x_{\gamma_{1}, \gamma_{2}}$ is harmonic. By Lemma 8.2.1 
and Corollary 8.2 .2 we have

$$
\begin{aligned}
& E x_{\gamma_{1}, \gamma_{2}}=-1 / 2\left[1-\left(2 \delta, S_{\gamma_{2}} \gamma_{1}\right)+\left(S_{\gamma_{2}} \gamma_{1}, s_{\gamma_{2}} \gamma_{1}\right)\right] \bar{e} \gamma_{2} \otimes \bar{e}_{s_{\gamma_{2}} \gamma_{1}}
\end{aligned}
$$

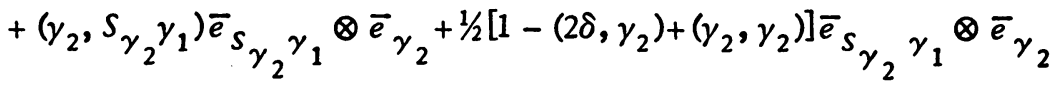

$$
\begin{aligned}
& -\left(\gamma_{2}, s_{\gamma_{2}} \gamma_{1}\right) \bar{e}_{\gamma_{2}} \otimes \bar{e}_{s_{\gamma_{2}} \gamma_{1}}
\end{aligned}
$$

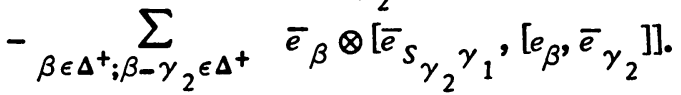

$$
\begin{aligned}
& \beta-s_{\gamma_{2}} \gamma_{1}-\gamma_{2} \in \Delta^{-}
\end{aligned}
$$

Now if $\beta \epsilon \Delta^{+}$such that $\beta-\gamma_{2} \epsilon \Delta^{+}$and $\beta-S_{\gamma_{2}} \gamma_{1}-\gamma_{2} \epsilon \Delta^{-}$, then $\beta+\beta_{1}=$ $\gamma_{2}+S_{\gamma_{2}} \gamma_{1}$ for some $\beta_{1} \in \Delta^{+}$and $\beta \neq \gamma_{2}$. By Lemma 8.7.1 we must have $\beta=$ $s_{\gamma_{2}} \gamma_{1}$ and $\beta_{1}=\gamma_{2}$. Therefore

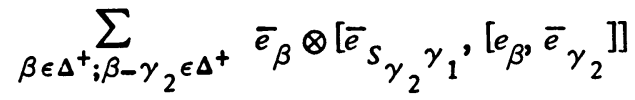

$$
\begin{aligned}
& \beta-s_{\gamma_{2}} \gamma_{1}-\gamma_{2} \epsilon \Delta^{+} \\
& =\bar{e} s_{\gamma_{2}} \gamma_{1} \otimes\left[e_{\gamma_{2}} \gamma_{1},\left[e_{\left.\left.s_{\gamma_{2}} \gamma_{1},{ }^{\bar{e}} \gamma_{2}\right]\right]}\right]\right. \\
& =\bar{e}_{s_{\gamma_{2}} \gamma_{1} \otimes\left(\gamma_{2}, s_{\gamma_{1}} \gamma_{1}\right) \bar{e}} \gamma_{2}=-\left(\gamma_{1}, \gamma_{2}\right) \bar{e}_{s_{\gamma_{2}} \gamma_{1} \otimes \bar{e}_{\gamma_{2}}}
\end{aligned}
$$

by the Jacobi identity since $\gamma_{2}+S_{\gamma_{2}} \gamma_{1}$ is not a root. Therefore since $S_{\gamma_{2}}$ is orthogonal

$$
\begin{aligned}
E x_{\gamma_{1}, \gamma_{2}=} & -1 / 2\left[1+2\left(\gamma_{1}, \gamma_{2}\right)\right] \bar{e} \gamma_{2} \otimes \bar{e} s_{\gamma_{2}} \gamma_{1} \\
& -\left(\gamma_{1}, \gamma_{2}\right) \bar{e} s_{\gamma_{2}} \gamma_{1} \otimes \bar{e} \gamma_{2}+1 / 2 \bar{e} s_{\gamma_{2}} \gamma_{1} \otimes \bar{e} \gamma_{2} \\
& +\left(\gamma_{1}, \gamma_{2}\right) \bar{e} \gamma_{2} \otimes \bar{e} s_{\gamma_{2}} \gamma_{1}+\left(\gamma_{1}, \gamma_{2}\right) \bar{e} s_{\gamma_{2}} \gamma_{1} \otimes \bar{e} \gamma_{2} \\
= & -1 / 2 x_{1}, \gamma_{2} \cdot
\end{aligned}
$$

On the other hand

$$
-c\left(-\gamma_{2}-s_{\gamma_{2}} \gamma_{1}\right)=-1 / 2\left[1+\left(2 \delta, \gamma_{2}+s_{\gamma_{2}} \gamma_{1}\right)-\left|\gamma_{2}+s_{\gamma_{2}} \gamma_{1}\right|^{2}\right]=-1 / 2
$$

by (7.4.12) and Lemma 8.7.1. Therefore

$$
E x_{\gamma_{1}, \gamma_{2}}=-1 / 2 x_{\gamma_{1}, \gamma_{2}}=-c\left(-\gamma_{2}-S_{\gamma_{2}} \gamma_{1}\right) x_{\gamma_{1}, \gamma_{2}}
$$

implies $x_{\gamma_{1}, \gamma_{2}}$ is harmonic; see (8.2.5). We can now present 
Theorem 8.7.13 Suppose $g$ is simple with rank $l$. Let 5 be a Cartan subalgebra of $g$ such that $\mathfrak{G}=\bar{\zeta}\left(\right.$ see (2.2.2)) and let $n=\Sigma_{a \in \Delta^{+}} g_{a}$ be the nilpotent subalgebra of 9 generated by the positive root spaces. Let $n^{\prime}$ be the complex dual space of $n$. $n^{\prime}$ is an $n$ module under the co-adjoint action. The first derived cohomology space $H^{1}\left(n, n^{\prime}\right)$ has dimension $l^{2}+l-1$ (over $\mathrm{C}$ ) and as an 5 module

$$
\begin{aligned}
& H^{1}\left(n, n^{\prime}\right)=\sum_{\alpha \in \pi} \mathrm{C} \bar{e}_{\alpha} \otimes \bar{e}_{\alpha^{+}}+\sum_{\substack{\text { ordered pairs } \\
(a, \beta) \in \pi \times \pi ; \alpha+\beta \epsilon \Delta^{+}}} \mathrm{C}\left(\bar{e}_{\alpha} \otimes \bar{e}_{\beta}+\bar{e}_{\beta} \otimes \bar{e}_{\alpha}\right) \\
& +\sum_{\substack{\text { ordered pairs } \\
(a, \beta) \epsilon \pi \times \pi ;(a, \beta)=0}}\left(\mathrm{C} \bar{e}_{\alpha} \otimes \bar{e}_{\beta}+\mathrm{C} \bar{e}_{\beta} \otimes \bar{e}_{\alpha}\right) \\
& \sum_{\text {distinct (unordered) pairs }} \mathrm{C} x_{\alpha, \beta} \\
& (\alpha, \beta) \epsilon \pi \times \pi ;(\alpha, \beta) \neq 0
\end{aligned}
$$

where $\pi$ is a system of simple roots and

(i) $x_{a, \beta}=\bar{e}_{\beta} \otimes \bar{e}_{s_{\beta} a}-\bar{e}_{s_{\beta} a} \otimes \bar{e}_{\beta}$ for $\alpha, \beta$ in $\pi$, where $S_{\beta}$ is the simple Weyl reflection determined by $\beta$ (see (4.2.11));

(ii) $\left\{e_{\beta}\right\}_{\beta \epsilon \Delta^{+}},\left\{\bar{e}_{\beta}\right\}_{\beta \epsilon \Delta^{+}}$are orthonormal bases of $n, \bar{n}=\Sigma_{\beta \epsilon \Delta^{+}}{ }^{g}-\beta$ (as in Theorem 7.5.3); see (2.2.1).

Proof. By (8.1.4), $H^{1}\left(n, n^{\prime}\right)=\Sigma_{\xi}\left(\Lambda^{1} \bar{n} \otimes \bar{n}\right)_{0}^{\xi}$ (direct sum) where $\xi$ varies over the distinct weights $-\alpha-\beta, \alpha, \beta \in \Delta^{+}$. Among the weights $-\alpha-\beta$ such that $\alpha+\beta$ is a root, only those for which both $\alpha, \beta \in \pi$ contribute to cohomology and this contribution is the summand $\mathrm{C}\left(\bar{e}_{\alpha} \otimes \bar{e}_{\beta}+\bar{e}_{\beta} \otimes \bar{e}_{\alpha}\right)$; see Proposition 8.5.1 and Proposition 8.5.6. The number of such summands is $l-1$ by Proposition 7.5.4.

Now consider weights $-\alpha-\beta, \alpha, \beta \in \Delta^{+}$, such that $\alpha+\beta$ is not a root. Among these which satisfy $|\alpha+\beta|^{2} \neq(a+\beta, 2 \delta)$ only those of the form $-2 \gamma$, $\gamma \in \pi$, contribute to cohomology. The contribution is the $l$ summands $\mathrm{C}_{\bar{\gamma}} \otimes \bar{e}_{\gamma}$, $\gamma \in \pi$; see Proposition 8.6.1. Finally consider weights $-\alpha-\beta, \alpha, \beta \in \Delta^{+}$for which $|\alpha+\beta|^{2}=(\alpha+\beta, 2 \delta)$. By Proposition 8.7.10 for each ordered pair of distinct simple roots $\{\alpha, \beta\}$ for which $(\alpha, \beta)=0$ (i.e. for which $\alpha+\beta$ is not a root), there is the contribution $\mathrm{C} \bar{e}_{\alpha} \otimes \bar{e}_{\beta}+\mathrm{C} \bar{e}_{\beta} \otimes \bar{e}_{a}$ to cohomology. The number of such pairs is $(l-1)(l-2) / 2$, as is easily seen by an examination of the Cartan matrices. The contribution of such pairs to the dimension of $H^{1}\left(n, n^{\prime}\right)$ is therefore $(l-1)(l-2)$. On the other hand if $\alpha, \beta$ are distinct simple roots, then $(\alpha, \beta) \neq 0$ if and only if $S_{\beta} \alpha$ is not simple. Therefore by Lemma 8.7.1 and Proposition 8.7.11 the remaining contributions to cohomology are the summands $C x_{a, \beta}$ (see 8.7.12) where $\alpha, \beta$ are distinct simple roots such that $(\alpha, \beta) \neq 0$. The number of such (unordered) pairs is the number of nonzero off-diagonal elements 
in the Cartan matrix of $g$. Thus this number is $l^{2}$ - number of diagonal elements twice the number of zeroes above the diagonal. But we have just seen that the number of zeroes above the diagonal is $(l-1)(l-2) / 2$; this is the number of ordered pairs of distinct simple roots $\{\mu, \nu\}$ for which $(\mu, \nu)=0$. Therefore there are $l^{2}-l-$ $(l-1)(l-2)=2(l-1)$ summands $\mathbf{C} x_{a, \beta}$. We now see moreover that

$$
\operatorname{dim} H^{1}\left(n, n^{\prime}\right)=l-1+l+(l-1)(l-2)+2(l-1)=l^{2}+l-1 .
$$

Note that $H^{1}\left(n, n^{\prime}\right)$ and $H^{1}(n, g / n)$ have the same dimension.

Remarks on $H^{2}(n, n)$. The nature of the cohomology groups $H^{2}(n, n)$ could now be determined with a modest amount of additional labor. One can see for example that

$$
0 \rightarrow H^{1}(n, g / n) \rightarrow H^{2}(n, n) \rightarrow H^{2}(n, g) \rightarrow 0
$$

is exact when $l>2, l=$ rank of $g$.

Now $\operatorname{dim} H^{2}(n, g)=(l-1)(l+2) / 2$ by Theorem 5.14 of [6]. Hence

$$
\operatorname{dim} H^{2}(n, n)=\operatorname{dim} H^{1}(n, g / n)+\operatorname{dim} H^{2}(n, g)=1 / 2\left(3 l^{2}+3 l-4\right)
$$

for $l>2$; see Theorem 7.5.3. The author has learned of the interesting work of Leger and Luks [8] which contains, among other results, a complete account of $H^{2}(n, n)$. In regard to the sequence in (8.7.14) they have observed the following deeper result:

Theorem (Leger and Luks). If the rank of $g$ is greater than $j$ then is an exact sequence.

$$
0 \rightarrow H^{j-1}(n, g / n) \rightarrow H^{j}(n, n) \rightarrow H^{j}(n, g) \rightarrow 0
$$

\section{REFERENCES}

1. P. Cartier, Remarks on "Lie algebra cohomology and the generalized Borel-Weil theorem" by B. Kostant, Ann. of Math. (2) 74 (1961), 388-390. MR 26 \#267.

2. P. Griffiths and W. Schmid, Locally homogeneous complex manifolds, Acta Math. 123 (1969), 253-302. MR $41 \# 4587$.

3. G. Hochschild and J.-P. Serre, Cohomology of Lie algebras, Ann. of Math. (2) 57 (1953), 591-603. MR 14, 943.

4. N. Iwahori, On the structure of a Hecke ring of a Chevalley group over a finite field, J. Fac. Sci. Univ. Tokyo Sect. I 10 (1964), 215-236. MR 29 \#2307.

5. N. Jacobson, Lie algebras, Interscience Tracts in Pure and Appl. Math., no. 10, Interscience, New York, 1962. MR $26 \# 1345$.

6. B. Kostant, Lie algebra cohomology and the generalized Borel-Weil theorem, Ann. of Math. (2) 74 (1961), 329-387. MR $26 \# 265$.

7. J.-L. Koszul, Homologie et cohomologie des algèbres de Lie, Bull. Soc. Math. France 78 (1950), 65-127. MR 12, 120.

8. G. Leger and E. Luks, Cohoinology of nilradicals of Borel subalgebras, Trans. Amer. Math. Soc. 195 (1974), 305-316.

DEPARTMENT OF MATHEMATICS, MASSACHUSETTS INSTITUTE OF TECHNOLOGY, CAMBRIDGE, MASSACHUSETTS 02139 